NBER WORKING PAPER SERIES

\title{
LIFE INSURANCE AND LIFE SETTLEMENT MARKETS WITH OVERCONFIDENT POLICYHOLDERS
}

\author{
Hanming Fang \\ Zenan $\mathrm{Wu}$ \\ Working Paper 23286 \\ http://www.nber.org/papers/w23286 \\ NATIONAL BUREAU OF ECONOMIC RESEARCH \\ 1050 Massachusetts Avenue \\ Cambridge, MA 02138 \\ March 2017
}

We would like to thank Douglas Bernheim, Yongmin Chen, Jiong Gong, Daniel Gottlieb, Michael Grubb, Glenn Harrison, Daniel Houser, Botond K szegi, Jianpei Li, Sherry Li, Jaimie Lien, Andrew Postlewaite and John Quah, as well as participants at Tsinghua Conference on Theoretical and Behavioral Economics (2015), UIBE IO and Competition Policy Workshop (2016), UNSW Superannuation Symposium (2016) for useful comments and discussions. Fang would also like to gratefully acknowledge the generous financial support from the National Science Foundation through Grant SES-0844845. Wu thanks School of Economics, Peking University for research support. All remaining errors are our own. The views expressed herein are those of the authors and do not necessarily reflect the views of the National Bureau of Economic Research.

NBER working papers are circulated for discussion and comment purposes. They have not been peer-reviewed or been subject to the review by the NBER Board of Directors that accompanies official NBER publications.

(C) 2017 by Hanming Fang and Zenan Wu. All rights reserved. Short sections of text, not to exceed two paragraphs, may be quoted without explicit permission provided that full credit, including $\odot$ notice, is given to the source. 
Life Insurance and Life Settlement Markets with Overconfident Policyholders

Hanming Fang and Zenan $\mathrm{Wu}$

NBER Working Paper No. 23286

March 2017

JEL No. D03,D86,G22,L11

\begin{abstract}
$\underline{\text { ABSTRACT }}$
We analyze how the life settlement market - the secondary market for life insurance - may affect consumer welfare in a dynamic equilibrium model of life insurance with one-sided commitment and overconfident policyholders. As in Daily et al. (2008) and Fang and Kung (2010), policyholders may lapse their life insurance policies when they lose their bequest motives; but in our model the policyholders may underestimate their probability of losing their bequest motive, or be overconfident about their future mortality risks. For the case of overconfidence with respect to bequest motives, we show that in the absence of life settlement overconfident consumers may buy "too much" reclassification risk insurance for later periods in the competitive equilibrium. In contrast, when consumers are overconfident about their future mortality rates in the sense that they put too high a subjective probability on the low-mortality state, the competitive equilibrium contract in the absence of life settlement exploits the consumer bias by offering them very high face amounts only in the low-mortality state. In both cases, life settlement market can impose a discipline on the extent to which overconfident consumers can be exploited by the primary insurers. We show that life settlement may increase the equilibrium consumer welfare of overconfident consumers when they are sufficiently vulnerable in the sense that they have a sufficiently large intertemporal elasticity of substitution of consumption.
\end{abstract}

\author{
Hanming Fang \\ Department of Economics \\ University of Pennsylvania \\ 3718 Locust Walk \\ Philadelphia, PA 19104 \\ and NBER \\ hanming.fang@econ.upenn.edu \\ Zenan Wu \\ School of Economics \\ Peking University \\ Beijing, China \\ zenan@pku.edu.cn
}




\section{Introduction}

Life insurance is a prevalent long-term contract for people to keep their dependents from economic disaster when the policyholders die. Life insurance is a large and growing industry. According to Life Insurance Marketing and Research Association International (LIMRA international), 70\% of U.S. households owned some type of life insurance in 2010. U.S. families purchased $\$ 2.8$ trillion of insurance coverage in 2013 and the total life insurance coverage in the US was $\$ 19.7$ trillion by the end of 2013. The average face amount of the individual life insurance policies purchased increased from $\$ 81$ thousand in 1993 to $\$ 165$ thousand in 2013 at an average annual growth rate of $3.56 \%$, 1

An important feature of the life insurance market is that policyholders lapse their policies before the period of coverage and receive the cash surrender value (CSV) that is a substantially small fraction (typically 3-5\%) of the policy's face value $2^{2}$ Policyholders may lapse the contract if they no longer need life insurance (e.g., loss of their bequest motives) or if they are pressed for liquidity (e.g., due to a negative income shock, or a large unexpected medical expenditure shock). Recently, the secondary market for life insurance - life settlement market - emerges and offers the policyholders the option of selling their unwanted policy for more than the CSV. Fang and Kung (2012) show that income shocks are relatively more important than bequest motive in explaining lapsation when policyholders are young. As policyholders age, bequest motive shocks become more important. Since the candidates for life settlements are "typically 65 or older" (see the reference in Footnote 2), we will assume in this paper that the driver of the lapsation is the loss of bequest motives.

Although the life settlement market is in its infancy, it draws attentions from the life insurance firms who put intensive effort into lobbying to prohibit the securitization of life settlement contracts $3^{3}$ They argue that the life insurance contract is designed to take into consideration of the fact that a fraction of policyholders lapse the contract without receiving the death benefit. The existence of the settlement market forces the insurance firms to pay death benefits on more policies than expected, which will lead to higher premiums for policyholders in the long run and hurt consumers eventually. The life settlement industry has been working hard to justify its existence, emphasizing its role of enhancing liquidity to policyholders.4 It is interesting to note that the life settlement industry has gained some success recently. For instance, in 2010, the General Assembly in Kentucky passed a bill requiring insurers to inform policyholders who are considering surrendering their policy that the settlement is a potential alternative. 5

Should the life settlement industry be banned? To resolve this theoretically and empirically

\footnotetext{
${ }^{1}$ See American Council of Life Insurers $(2014)$.

${ }^{2}$ See http://www.lisa.org/content/13/What-is-a-Life-Settlement.aspx/

${ }^{3}$ See Martin 2010) for detailed discussions of life insurance and life settlement market.

${ }^{4}$ As mentioned in Martin (2010): "In 2008, the executive of the life settlement industry's national trade organization testifies to the Florida Office of Insurance Regulation that the 'secondary market for life insurance has brought great benefits to consumers, unlocking the value of life insurance policies."'

${ }^{5}$ Similar requirements exist in Maine, Oregon, Washington (See Martin, 2010) and U.K (See Januário and Naik, 2014 ).
} 
important question, it would be useful to understand the role of the life settlement market and its impact on policyholders' welfare. In this paper we extend the models of Daily et al. $(2008)$ and Fang and Kung (2010) to study the welfare implication of the life settlement market by assuming that consumers are biased. Specifically, consumers may be overconfident about either the probability of losing their bequest motives at the time they purchase the contract, or about their future mortality risks. ${ }^{6}$ Daily et al. (2008) are aware of the importance of the investigation of the consequences of consumer's behavioral bias in life insurance market, as they stated: "In our model, all agents are forward-looking and standard-expected-utility maximizers. We thus abstract from potential violations of 'rational' behavior. Our view is that considerations such as regret or misperceptions of probabilities may very well be relevant in this [life insurance] market."

We show that, when life insurance policyholders exhibit overconfidence either of their bequest motives or mortality risks, the presence of the settlement market provides them a channel to correct their earlier mistakes and mitigate the loss due to their misperception. This new role of the settlement market generates a potential welfare gain which is absent when consumers are fully rational; and indeed we show that life settlement can potentially increase consumer welfare in equilibrium. In contrast, Daily et al. (2008) and Fang and Kung (2010) show that, when consumers have rational beliefs and when lapsation is due to loss of bequest motive, introducing life settlement market always reduces consumer welfare in equilibrium. Thus our results contribute to the debate over the potential welfare consequences of life settlement market.

This paper is related to the growing life insurance literature. In a seminal paper, Hendel and Lizzeri (2003) use a two-period model to analyze the role of commitment on long-term life insurance contract. In their model, risk-neutral life insurance firms compete to offer contracts to risk averse consumers who are subject to mortality risk. Consumers' health status may change over time and thus face reclassification risk. Insurance firms are able to commit to contractual terms while consumers can lapse the contract in the second period, lacking commitment power (i.e., one-sided commitment). They prove that the equilibrium contract is front-loaded: consumers are offered a contract with first period premium that is higher than actuarially fair in exchange for reclassification risk in the second period. Daily et al. (2008) and Fang and Kung (2010) investigate this problem further by introducing a settlement market and analyze its effect on the equilibrium contract and consumer welfare. In their models, policyholders may lose bequest motive in the second period, facilitating lapsation and the demand for the settlement market. Using a model similar to Hendel and Lizzeri (2003), Fang and Kung (2010) show that the shape of the equilibrium contract is fundamentally changed in the presence of the settlement market. Instead of flat premiums, a contract with premium discounts is offered in the second period. They conclude that consumer welfare is reduced in the presence of the settlement market. In recent independent research, Gottlieb

\footnotetext{
${ }^{6}$ Many studies document that people are unrealistically optimistic about future life events. For instance, Weinstein (1980) finds strong evidence of over-optimism in a lab experiment setting with 258 college students. Subjects overwhelmingly predict themselves to be better than a median individual regarding positive events and below average regarding negative events. Robb et al. (2004) also detects underestimation of risk among patients who participated in cancer examination. They find that the self perceived risk is lower than the actual risk of colorectal cancer determined by flexible sigmoidoscopy screening.
} 
and Smetters (2014) investigate the equilibrium life insurance contracts where lapsation is motivated by a negative income shock. Similar to our paper, consumers are overconfident in the sense that they place zero probability on the event of experiencing the liquidity shock. Unlike Hendel and Lizzeri (2003), the equilibrium contract is front-loaded because front-loading makes the policy more attractive to policyholders with misperception of the probability of lapsing the contract. They show that the presence of the settlement market would increase consumer welfare if lapsation is due to liquidity shock.

This paper also belongs to the strand of literature on behavioral contract theory 7 Most papers assume consumers exhibit some type of behavioral bias and investigate how firms design contracts accordingly. For instance, de la Rosa (2011) and Santos-Pinto (2008) study the incentive contract in a principal-agent model of moral hazard when agent is overconfident. Fang and Moscarini (2005) show that firms will have incentives to compress wages so as not to reveal its private information about workers' productivity in an environment where workers are overconfident about their ability and ability and effort are complements in the production function. Grubb (2009) proposes a model of contracting overconfident consumers in US cellular phone services market, and confirms evidence of overconfidence. In the context of insurance market, Sandroni and Squintani (2007) modify the textbook Rothschild-Stiglitz model to study the equilibrium contract by assuming that part of the insurees are overconfident about their risk types. They find that when a significant fraction of individuals are overconfident, compulsory insurance serves as a transfer of income between different types of agents. Their results have much different implications than Rothschild and Stiglitz (1976) on government intervention in insurance market. Spinnewijn (2015) studies the optimal unemployment insurance contract under perfect competition where the insuree has misperception on the probability of finding a job. Gottlieb (2008) considers the impact of non-exclusivity in a competitive market when firms offer contracts to compete for the business of present-biased consumers. He shows that non-exclusive contracts would invalidate the type of profit-maximizing contracts proposed in Dellavigna and Malmendier (2006) for leisure goods - goods with immediate rewards and deferred costs, e.g. tobacco, alcohol, and unhealthy food - where present-biased consumers would receive a lump-sum transfer and pay a usage price higher than the marginal cost. In some sense, the non-exclusivity of the contracts in Gottlieb (2008) plays a role similar to the secondary market in our setting. Our paper contributes to this strand of literature by pointing out the role of a secondary market in alleviating the negative consequences caused by consumers' behavioral bias.

The remainder of the paper is organized as follows. In Section 2 , we present the baseline model of dynamic life insurance without the life settlement market when policyholders underestimate the probability of losing their bequest motive, and characterize the set of equilibrium contracts. In Section 3, we incorporate the settlement market into the baseline model. In Section 4, we study the welfare consequences of introducing the settlement market, and state one of our main results (Proposition 7). In Section 5, we consider the situation where policyholders exhibit overconfidence of future mortality risk, and show that our results derived in Section 4 are robust (Proposition 8).

\footnotetext{
${ }^{7}$ See Köszegi 2014) for a comprehensive survey on this topic, and also Grubb 2015) for a survey of the literature on overconfident consumers in the marketplace.
} 
In Section 6, we summarize our main findings and suggest directions for future research. All proofs are presented in the Appendix.

\section{The Baseline Model without the Settlement Market}

In this section, we propose a model of dynamic life insurance slightly modified from Hendel and Lizzeri (2003), Daily et al. (2008) and Fang and Kung (2010), and introduce consumer bias on the prediction of the probability of losing their bequest motives. We will discuss overconfidence of the future mortality risks in Section 5 .

\subsection{The Model}

Consider an environment with a continuum of consumers (potential policyholders) who may live up to two periods. The life insurance market is perfectly competitive.

Income, health and preference. The policyholder receives an income of $y-g$ in the first period and $y+g$ in the second period, where $g \in[0, y)$ is a measure of income growth. In the first period, the policyholder has a death probability of $p_{1} \in(0,1)$, which is common knowledge between policyholders and insurance firms. The death probability is interpreted as the health status of the policyholder. In the second period, the mortality risk $p_{2} \in[0,1]$ is randomly drawn from a distribution with continuous density $\phi(\cdot)$ and corresponding CDF $\Phi(\cdot)$. Health status $p_{2}$ is not known in the first period when the policyholder purchases the insurance and is symmetrically learned by the insurance firms and the policyholder at the beginning of the second period.

A policyholder has two potential sources of utility. If the policyholder is alive and consumes $c \geq 0$, he receives utility $u(c)$ from his own consumption; if the policyholder dies, then he receives utility $v(c)$ from his dependent's consumption $c$, provided that the policyholder retains his bequest motive at the time of his death. We assume that both $u(\cdot)$ and $v(\cdot)$ are strictly increasing, twice differentiable and strictly concave. Furthermore, we assume that $u(\cdot)$ and $v(\cdot)$ satisfy the Inada conditions: $\lim _{c \rightarrow 0} u^{\prime}(c)=\infty, \lim _{c \rightarrow 0} v^{\prime}(c)=\infty$ and $\lim _{c \rightarrow \infty} v^{\prime}(c)=0$.

Bequest motives and overconfidence. A policyholder does not lose his bequest motive in the first period. However, the policyholder may lose his bequest motive with probability $q \in(0,1)$ at the beginning of period 2. If the policyholder loses his bequest motive, then he no longer derives utility from his dependent's consumption, in which case he receives some constant utility normalized to zero if he dies.

The policyholder believes his probability of losing bequest motive is $\tilde{q} \in[0, q]$. When $\tilde{q}=q$, the policyholder is rational and the model degenerates to Fang and Kung (2010). When $\tilde{q}<q$, the policyholder exhibits overconfidence in the sense that he underestimates the probability of losing his bequest motive. Both $\tilde{q}$ and $q$ are assumed to be common knowledge. 
For the ease of our exposition, we will denote

$$
\Delta \equiv \frac{q-\tilde{q}}{q}
$$

as the degree of the consumer's overconfidence. In particular, when $\Delta=0$, policyholder is fully rational and forms correct belief about $q$. When $\Delta=1$, a policyholder is extremely overconfident and never expects himself to lose bequest motive in the second period. When there is a continuum of consumers, the variable $\Delta \in[0,1]$ also indicates the fraction of policyholders that lose bequest motives unexpectedly in period 2 .

Timing, commitment and contracts. At the beginning of the first period, the consumer learns his period-1 health status $p_{1}$ and then chooses to purchase a long-term life insurance contract. As in Hendel and Lizzeri (2003), a long-term insurance contract is in the form of 8

$$
\left\langle\left(Q_{1}, F_{1}\right),\left(Q_{2}\left(p_{2}\right), F_{2}\left(p_{2}\right)\right): p_{2} \in[0,1]\right\rangle
$$

where $\left\langle Q_{1}, F_{1}\right\rangle$ specifies a premium and face value for the first period, and $\left\langle Q_{2}\left(p_{2}\right), F_{2}\left(p_{2}\right)\right\rangle$ specifies the corresponding premium and face value for each health status $p_{2} \in[0,1]$ in the second period. Note that the second period premium and face value are state dependent.

At the end of the first period, with probability $p_{1}$, the policyholder dies and his dependents receives the face value $F_{1}$. With the remaining probability, the policyholder continues to period 2 and observes, as does the insurance company, his period-2 health status $p_{2}$. We assume one-sided commitment by the insurance firm, that is the insurance firm can commit to future premiums and face values specified in the long-term contract. However, the policyholder can choose to continue with the long-term contract purchased in the first period, but he is also free to terminate the longterm contract purchased in period 1 and purchase a spot contract from the perfectly competitive spot market if he desires. When we later introduce life settlement market, he can also sell the long-term contract in the secondary market.

\subsection{Equilibrium Contracts}

We first characterize the equilibrium contract without the settlement market. The key here is to characterize how the competitive insurers will design their dynamic long-term contracts so as to most appeal to the overconfident consumers. The equilibrium long-term contract $\left\langle\left(Q_{1}, F_{1}\right),\left(Q_{2}\left(p_{2}\right)\right.\right.$,

\footnotetext{
${ }^{8}$ The contract terms should all be indexed by $(q, \tilde{q})$ or $(q, \Delta)$. We ignore this for expositional ease.
} 


$$
\begin{aligned}
\left.F_{2}\left(p_{2}\right)\right): p_{2} \in & {[0,1]\rangle \text { solves } !^{9} } \\
& \max \left[u\left(y-g-Q_{1}\right)+p_{1} v\left(F_{1}\right)\right] \\
& +\left(1-p_{1}\right) \int_{0}^{1}\left\{(1-\tilde{q})\left[u\left(y+g-Q_{2}\left(p_{2}\right)\right)+p_{2} v\left(F_{2}\left(p_{2}\right)\right)\right]+\tilde{q} u(y+g)\right\} d \Phi\left(p_{2}\right) \\
\text { s.t. } & \left(Q_{1}-p_{1} F_{1}\right)+\left(1-p_{1}\right)(1-q) \int_{0}^{1}\left[Q_{2}\left(p_{2}\right)-p_{2} F_{2}\left(p_{2}\right)\right] d \Phi\left(p_{2}\right)=0, \\
& Q_{2}\left(p_{2}\right)-p_{2} F_{2}\left(p_{2}\right) \leq 0, \text { for all } p_{2} \in[0,1], \\
& Q_{2}\left(p_{2}\right) \geq 0, \text { for all } p_{2} \in[0,1] .
\end{aligned}
$$

Note that the objective function (3) in the above maximization problem is the policyholders' expected perceived utility using the subjective belief $\tilde{q}$ about losing bequest motive, instead of the utility based on the objective probability $q$ of losing bequest motive 10 Constraint (4) is the zeroprofit condition that captures the competition in the primary market. It is important to note that in (4), the insurance company uses the objective probability $q$ of the policyholder losing bequest motives in evaluating the second-period expected profit. Constraint (5) is the no-lapsation condition for policyholders whose bequest motives remain in period 2. The constraint (5) is important because the consumer is not committed to continue with the long-term contract, and he will not terminate the contract only if staying with the long-term contract is preferred to purchasing a spot contract. The intuition for why (5) ensures no lapsation for those with bequest motives is as follows ${ }^{11}$ For any long-term contract as specified by $(2), p_{2} F_{2}\left(p_{2}\right)-Q_{2}\left(p_{2}\right)$ is the actuarial value of the second-period contract in health state $p_{2}$. Due to one-sided commitment, the policyholder can opt for a spot contract in the second period. Since the spot market is perfectly competitive, the actuarial value of the spot contract must be zero. Thus in order to prevent the policyholder from lapsing the long-term contract and substituting it with a spot contract, the actuarial value of the second-period contract must be non-negative for all $p_{2}$, i.e., $p_{2} F_{2}\left(p_{2}\right)-Q_{2}\left(p_{2}\right) \geq 0$. Finally, constraint (6) simply states that the second period premium for any health status can not be negative.

Remark 1 The non-negativity constraint for $Q_{1}$ and $F_{1}$ are ignored. The non-negativity of $F_{1}$ never binds due to the Inada condition on $v(\cdot) ; Q_{1}$ will be strictly positive because constraints (5) and (4) require that $Q_{1}-p_{1} F_{1}$ must be non-negative.

Remark 2 The non-negativity constraints of the period-2 face values $F_{2}\left(p_{2}\right)$ are ignored because they will never bind due to the Inada condition on $v(\cdot)$.

\footnotetext{
${ }^{9}$ The maximization problem (3) is similar to that of Fang and Kung (2010, p. 6) with two main differences: first, the perceived probability $\tilde{q}$ of losing bequest motives is used in the objective function of the consumers; second, the non-negative constraints for second-period premiums (6) may be binding in this environment in contrast to the case when $\tilde{q}=q$, as we will show below in Lemma 4 .

${ }^{10}$ Thus, the perceived expected utility in (3) is the decision utility in the terminology of Kahneman et al. (1997). As we will explain later, policyholders' expected utility according to the correct, or objective, probability of losing bequest motive $q$, is used when we evaluate the consumer welfare, corresponding to the notion of experienced utility in Kahneman et al. (1997).

${ }^{i 1}$ For a formal argument of constraint [5), see Hendel and Lizzeri (2003).
} 
Remark 3 The set of feasible contracts defined by the constraints (4)-(6) does not depend on consumer's subject belief $\tilde{q}$.

The first order conditions for problem (3) with respect to $Q_{1}, F_{1}, Q_{2}\left(p_{2}\right) F_{2}\left(p_{2}\right)$ yield:

$$
\begin{gathered}
u^{\prime}\left(y-g-Q_{1}\right)=\mu, \\
v^{\prime}\left(F_{1}\right)=\mu \\
(1-\tilde{q}) u^{\prime}\left(y+g-Q_{2}\left(p_{2}\right)\right)=(1-q) \mu+\frac{\lambda\left(p_{2}\right)+\gamma\left(p_{2}\right)}{\left(1-p_{1}\right) \phi\left(p_{2}\right)}, \text { for all } p_{2} \in[0,1], \\
(1-\tilde{q}) v^{\prime}\left(F_{2}\left(p_{2}\right)\right)=(1-q) \mu+\frac{\lambda\left(p_{2}\right)}{\left(1-p_{1}\right) \phi\left(p_{2}\right)}, \text { for all } p_{2} \in[0,1],
\end{gathered}
$$

where $\mu, \lambda\left(p_{2}\right)$ and $\gamma\left(p_{2}\right)$ are the Lagrange multipliers for constraint (4), (5) and (6); moreover, $\mu>0, \lambda\left(p_{2}\right) \leq 0$ and $\gamma\left(p_{2}\right) \geq 0$ all need to satisfy the complementary slackness conditions:

$$
\begin{gathered}
\lambda\left(p_{2}\right)\left[Q_{2}\left(p_{2}\right)-p_{2} F_{2}\left(p_{2}\right)\right]=0, \text { for all } p_{2} \in[0,1], \\
\gamma\left(p_{2}\right) Q_{2}\left(p_{2}\right)=0, \text { for all } p_{2} \in[0,1] .
\end{gathered}
$$

The first order conditions $7 \mathrm{a})$ and $7 \mathrm{~b}$ imply that:

$$
u^{\prime}\left(y-g-Q_{1}\right)=v^{\prime}\left(F_{1}\right)
$$

In equilibrium in period 1, the marginal utility of policyholder's consumption is equal to the marginal utility of his dependent's consumption; this is referred to as the full-event first period insurance in Hendel and Lizzeri (2003) and Fang and Kung (2010). Analogously, it would also be useful to define the fair premium and face amount for the full-event second period insurance in health state $p_{2} \in[0,1]$, which we denote by $Q_{2}^{F I}\left(p_{2}\right)$ and $F_{2}^{F I}\left(p_{2}\right)$ respectively, as the solution to the following pair of equations:

$$
\begin{gathered}
u^{\prime}\left(y+g-Q_{2}^{F I}\left(p_{2}\right)\right)=v^{\prime}\left(F_{2}^{F I}\left(p_{2}\right)\right), \\
Q_{2}^{F I}\left(p_{2}\right)-p_{2} F_{2}^{F I}\left(p_{2}\right)=0 .
\end{gathered}
$$

This is indeed the equilibrium spot contract with health state $p_{2}$ in period $2{ }^{12}$ However, it should be clear, from the first order conditions $(7 \mathrm{c})-(7 \mathrm{~d})$, that whether analogous full-event insurance will be achieved in equilibrium in period 2 will depend on whether constraint (6) is binding in health state $p_{2}$ : if constraint (6) is non-binding, then we know that $\gamma\left(p_{2}\right)=0$ and then $(7 \mathrm{c})-(7 \mathrm{~d})$ imply (11) below that defines full-event insurance.

To characterize the equilibrium contracts, we follow Fang and Kung (2010) and divide the support of the second period health states $p_{2}$ into two subsets $\mathcal{B}$ and $\mathcal{N B}$ : for $p_{2} \in \mathcal{B}$, the no-

\footnotetext{
${ }^{12}$ The second period spot contract $\left\langle Q_{2}\left(p_{2}\right), F_{2}\left(p_{2}\right)\right\rangle$ solves max $u\left(y+g-Q_{2}\left(p_{2}\right)\right)+p_{2} v\left(F_{2}\left(p_{2}\right)\right)$ subject to $Q_{2}\left(p_{2}\right)-$ $p_{2} F_{2}\left(p_{2}\right)=0$, which leads to the same conditions as in 10 .
} 
lapsation constraint (5) binds; for $p_{2} \in \mathcal{N B}$, the no-lapsation constraint (5) does not bind.

Lemma 1 If $p_{2} \in \mathcal{B}$ and $p_{2}^{\prime} \in \mathcal{N B}$, then $p_{2}<p_{2}^{\prime}, Q_{2}\left(p_{2}\right) \leq Q_{2}\left(p_{2}^{\prime}\right)$ and $F_{2}\left(p_{2}\right) \geq F_{2}\left(p_{2}^{\prime}\right)$.

The proof is similar to that of Lemma 1 in Fang and Kung (2010). Lemma 1 indicates that there exists a threshold $p_{2}^{*}$ such that $p_{2} \in \mathcal{B}$ if $p_{2}<p_{2}^{*}$ and $p_{2} \in \mathcal{N B}$ if $p_{2}>p_{2}^{*}$.

Lemma 2 If there exists a health state $\tilde{p}_{2} \neq 0$ such that constraint $(\sqrt[6]{6})$ is binding, i.e., $Q_{2}\left(\tilde{p}_{2}\right)=0$, then it must be the case that (6) is binding, namely, $Q_{2}\left(p_{2}\right)=0$, for all $p_{2} \in[0,1]$.

Lemma 2 narrows down the set of period-2 equilibrium premiums to one of two possibilities: (i) $Q_{2}\left(p_{2}\right)>0$ for all $p_{2} \in(0,1]$; or, (ii) $Q_{2}\left(p_{2}\right)=0$ for all $p_{2} \in(0,1]$.

Under case (i), the constraint (6) is not binding, thus $\gamma\left(p_{2}\right)=0$ for all $p_{2} \in(0,1]$. Hence, the first order conditions $7 \mathrm{c}$ and $(7 \mathrm{~d})$ yield that, for all $p_{2} \in(0,1]$,

$$
u^{\prime}\left(y+g-Q_{2}\left(p_{2}\right)\right)=v^{\prime}\left(F_{2}\left(p_{2}\right)\right)
$$

therefore, the policyholder obtains full-event insurance in period 2 for all health states $p_{2}$.

Under case (ii), $Q_{2}\left(p_{2}\right)=0$ implies that the constraint (5) is not binding, thus $\lambda\left(p_{2}\right)=0$ for all $p_{2} \in(0,1]$. Combining the first order conditions $(7 \mathrm{~b})$ and $(7 \mathrm{~d})$, we must have:

$$
(1-\tilde{q}) v^{\prime}\left(F_{2}\left(p_{2}\right)\right)=(1-q) v^{\prime}\left(F_{1}\right) \text { for all } p_{2} \in(0,1]
$$

which implies that under case (ii), $F_{2}\left(p_{2}\right)$ must be a constant for all $p_{2} \in(0,1]$.

Now we consider the implications of Lemmas 1 12 As we mentioned, Lemma 1 implies that there exists a threshold death probability $p_{2}^{*}$ in period 2 that divides the set $\mathcal{B}$ from $\mathcal{N} \mathcal{B}$. There are three possibilities: (i) $p_{2}^{*}=1$; (ii) $p_{2}^{*}=0$; (iii) $p_{2}^{*} \in(0,1)$.

First, consider the case of $p_{2}^{*}=1$. This implies that the no-lapsation condition (5) binds for all period-2 health states, i.e., $Q_{2}\left(p_{2}\right)-p_{2} F_{2}\left(p_{2}\right)=0$ for all $p_{2} \in(0,1]$. Since the Inada condition on $v(\cdot)$ implies that, in equilibrium, $F_{2}\left(p_{2}\right)>0$ for all $p_{2} \in(0,1]$. Therefore $Q_{2}\left(p_{2}\right)=p_{2} F_{2}\left(p_{2}\right)>0$ for all $p_{2} \in(0,1]$, which in turn implies that condition (11) also holds for all $p_{2} \in(0,1]$. Thus, in this case, the equilibrium period- 2 contract must correspond to the fair premium and face amount full-event spot insurance contracts defined by 10a - 10b.

Second, consider the case of $p_{2}^{*}=0$. We first argue that $p_{2}^{*}=0$ implies that:

$$
Q_{2}\left(p_{2}\right)=0 \text { for all } p_{2} \in(0,1]
$$

To see this, suppose to the contrary that $Q_{2}\left(p_{2}\right)>0$ for some $p_{2} \in(0,1]$; then by Lemma 2 , it must be that $Q_{2}\left(p_{2}\right)>0$ for all $p_{2} \in(0,1]$. Hence (11) holds. Moreover, by Lemma 1, $p_{2}^{*}=0$ implies that $p_{2} \in \mathcal{N B}$ for all $p_{2} \in(0,1]$, hence $\lambda\left(p_{2}\right)=0$ for all $p_{2} \in(0,1]$. Thus, the first order conditions (7) imply that

$$
u^{\prime}\left(y+g-Q_{2}\left(p_{2}\right)\right)=v^{\prime}\left(F_{2}\left(p_{2}\right)\right)=\frac{1-q}{1-\tilde{q}} u^{\prime}\left(y-g-Q_{1}\right) \text { for all } p_{2} \in(0,1] .
$$


Thus, $F_{2}\left(p_{2}\right)$ and $Q_{2}\left(p_{2}\right)$ must be constant for all $p_{2} \in(0,1]$. However, when $p_{2}$ is sufficiently small, $Q_{2}\left(p_{2}\right)-p_{2} F_{2}\left(p_{2}\right)$ must be strictly positive, contradicting (5). Therefore, if $p_{2}^{*}=0$, then the equilibrium contracts is fully characterized by (4), (9), (12) and (13).

Third, consider the case of $p_{2}^{*} \in(0,1)$. If $p_{2}<p_{2}^{*}$, the no-lapsation constraint (5) binds, which implies that constraint (6) is not binding, and hence $\gamma\left(p_{2}\right)=0{ }^{13}$ Thus, $7 \mathrm{c}$ and $7 \mathrm{~d}$ ) implies $u^{\prime}\left(y+g-Q_{2}\left(p_{2}\right)\right)=v^{\prime}\left(F_{2}\left(p_{2}\right)\right)$ for $p_{2}<p_{2}^{*}$. This, together with the binding constraint (5), implies that $\left\langle Q_{2}\left(p_{2}\right), F_{2}\left(p_{2}\right)\right\rangle=\left\langle Q_{2}^{F I}\left(p_{2}\right), F_{2}^{F I}\left(p_{2}\right)\right\rangle$ for all $p_{2}<p_{2}^{*}$ where $\left\langle Q_{2}^{F I}\left(p_{2}\right), F_{2}^{F I}\left(p_{2}\right)\right\rangle$ is characterized by (10). If $p_{2}>p_{2}^{*}$, then $p_{2} \in \mathcal{N B}$, hence $\lambda\left(p_{2}\right)=0$. Thus, the first order condition (7d) implies that $F_{2}\left(p_{2}\right)$ must be a constant. Moreover, from the discussion above for the case when $p_{2}<p_{2}^{*}$, we know that $Q_{2}\left(p_{2}\right)>0$ if $p_{2}<p_{2}^{*}$, hence Lemma 2 implies that $\gamma\left(p_{2}\right)=0$ for all $p_{2} \in(0,1]$. This implies that $u^{\prime}\left(y+g-Q_{2}\left(p_{2}\right)\right)=v^{\prime}\left(F_{2}\left(p_{2}\right)\right)$ for all $p_{2} \in(0,1]$. Since $F_{2}\left(p_{2}\right)$ is a constant for $p_{2}>p_{2}^{*}$, it must be that $Q_{2}\left(p_{2}\right)$ is a constant for $p_{2}>p_{2}^{*}$ as well and moreover, the premiums are front-loaded in the sense that $Q_{2}\left(p_{2}\right)<Q_{2}^{F I}\left(p_{2}\right)$ if $p_{2}>p_{2}^{*}$. That is, the insurance firms charge the policyholders a level period 2 premium for health states $p_{2}>p_{2}^{*}$ below the corresponding fair premium, so as to insure the policyholders against reclassification risk. Next, we characterize the equilibrium contract at $p_{2}=p_{2}^{*}$ :

Lemma 3 Suppose $p_{2}^{*} \in(0,1)$, then the equilibrium contract must satisfy the following at $p_{2}=p_{2}^{*}$ :

$$
\begin{aligned}
Q_{2}\left(p_{2}^{*}\right) & =Q_{2}^{F I}\left(p_{2}^{*}\right) \\
(1-\tilde{q}) u^{\prime}\left(y+g-Q_{2}^{F I}\left(p_{2}^{*}\right)\right) & =(1-q) u^{\prime}\left(y-g-Q_{1}\right) .
\end{aligned}
$$

The proof of Lemma 3 replicates that of Lemma 2 in Fang and Kung (2010) and is omitted. To summarize, when $p_{2}^{*} \in(0,1)$ the equilibrium period-2 premiums $Q_{2}\left(p_{2}\right)$ must satisfy

$$
Q_{2}\left(p_{2}\right)=\left\{\begin{array}{lll}
Q_{2}^{F I}\left(p_{2}\right) & \text { if } & p_{2} \leq p_{2}^{*} \\
Q_{2}^{F I}\left(p_{2}^{*}\right) & \text { if } & p_{2}>p_{2}^{*}
\end{array}\right.
$$

where $Q_{2}^{F I}\left(p_{2}\right)$ is uniquely determined by 10 . The equilibrium long-term contract in this case is fully characterized by (4), (9), (11) and (16).

Equation (15) also provides an explicit unique characterization for $p_{2}^{*}$ provided that $p_{2}^{*}$ lies strictly in $(0,1)$ since $Q_{2}^{F I}(\cdot)$ as defined by 10 is monotonically increasing. From Equation (15), it is clear that when $q$ is sufficiently close to 1 and $\tilde{q}$ is sufficiently close to 0 , the left hand side of Equation 15 will be higher than the right hand side even if $Q_{2}^{F I}\left(p_{2}^{*}\right)$ is 0 . When this occurs, $p_{2}^{*}$ will be 0 . The following lemma formalizes this result:

Lemma 4 There exists a threshold $\bar{q} \in(0,1)$ such that, for each $q \geq \bar{q}$ there exists a threshold $\bar{\Delta}(q) \in(0,1)$ such that $p_{2}^{*}=0$ if and only if $q \geq \bar{q}$ and $\Delta>\bar{\Delta}(q)$.

\footnotetext{
${ }^{13}$ Because $F_{2}\left(p_{2}\right)>0$ by the Inada condition on $v(\cdot)$, it follows immediately that $Q_{2}\left(p_{2}\right)=p_{2} F_{2}\left(p_{2}\right)>0$ for $p_{2} \in\left(0, p_{2}^{*}\right)$.
} 
The intuition for Lemma 4 is as follows. Since the insurance company only needs to pay out death benefit in period 2 if the policyholder retains his bequest motives, which occurs with probability $1-q$, the higher the $q$ is, the lower the cost for the insurance company to provide the policyholder reclassification risk insurance in period 2. Indeed the competitive market ensures that in equilibrium firms will be pushing $p_{2}^{*}$ lower so that more states have the level premiums. Importantly, if consumers are overconfident, then they subjectively value the period-2 reclassification risk insurance more than the objective cost for the firms to offer such insurance, thus creating a "wedge." Competitive pressure among the insurance firms to attract the consumers in the first period will force the insurance firms to exploit this wedge, thus pushing firms to offer more reclassification insurance in period 2, i.e., further pushing $p_{2}^{*}$ lower; of course, in order to break even, the firms will raise the period-1 premium. When these two effects are strong enough (i.e. for sufficiently high $q$ and $\Delta$ ), contracts with zero period-2 premiums can emerge in equilibrium.

The above discussions are summarized below:

Proposition 1 (Equilibrium Contracts without Life Settlement.) The equilibrium contracts in the absence of the life settlement market satisfy the following properties:

1. All policyholders receive full-event insurance in period 1.

2. There is a period-2 threshold health state $p_{2}^{*} \in[0,1]$ such that $p_{2} \in \mathcal{B}$ if $p_{2}<p_{2}^{*}$ and $p_{2} \in \mathcal{N B}$ if $p_{2}>p_{2}^{*}$.

3. (a) If $p_{2}^{*}=0$, all policyholders lose full-event insurance in period 2. The set of equilibrium contracts solves (4), (9), (12) and (13).

(b) If $0<p_{2}^{*}<1$, all policyholders receive full-event insurance in period 2. The set of equilibrium contracts solves (4), (9), (11) and (16).

(c) If $p_{2}^{*}=1$, the equilibrium contract coincides with spot contracts.

4. When $q$ and $\Delta$ are sufficiently large, $p_{2}^{*}=0$. Policyholders are fully insured against reclassification risk, receiving zero premiums in period 2 for all health states.

The wedge between the policyholder's subjective value of the period 2 reclassification risk insurance and the objective cost for the firms of offering such insurance is the key for the welfare effects of life settlement we will describe in Section 4. Note that this wedge is amplified as the consumers become more overconfident. The next result illustrates how the equilibrium contracts are affected by consumers' increasing overconfidence. Let us fix the objective probability of losing bequest motive $q \in(0,1)$. Consider two levels of subjective beliefs $\tilde{q}$ and $\widehat{\tilde{q}}$ such that $\hat{\Delta} \equiv(\widehat{\widetilde{q}}-q) / q<\Delta \equiv(\tilde{q}-q) / q$. Let $\left\langle\hat{Q}_{1}, \hat{F}_{1}, \hat{Q}_{2}\left(p_{2}\right), \hat{F}_{2}\left(p_{2}\right)\right\rangle$ and $\left\langle Q_{1}, F_{1}, Q_{2}\left(p_{2}\right), F_{2}\left(p_{2}\right)\right\rangle$ be the equilibrium long-term contracts under $\hat{\Delta}$ and $\Delta$ respectively, and let $\hat{p}_{2}^{*}$ and $p_{2}^{*}$ be the threshold probabilities characterized in Lemma 1 respectively under $\hat{\Delta}$ and $\Delta$. We have the following result:

Proposition 2 (Higher Overconfidence Exacerbates Front-Loading.) Fix $q \in(0,1)$. Suppose $\hat{\Delta}<\Delta$. If $0<\hat{p}_{2}^{*}<1$, then $\hat{F}_{1}>F_{1}, \hat{Q}_{1}<Q_{1}$ and $\hat{p}_{2}^{*}>p_{2}^{*}$. 


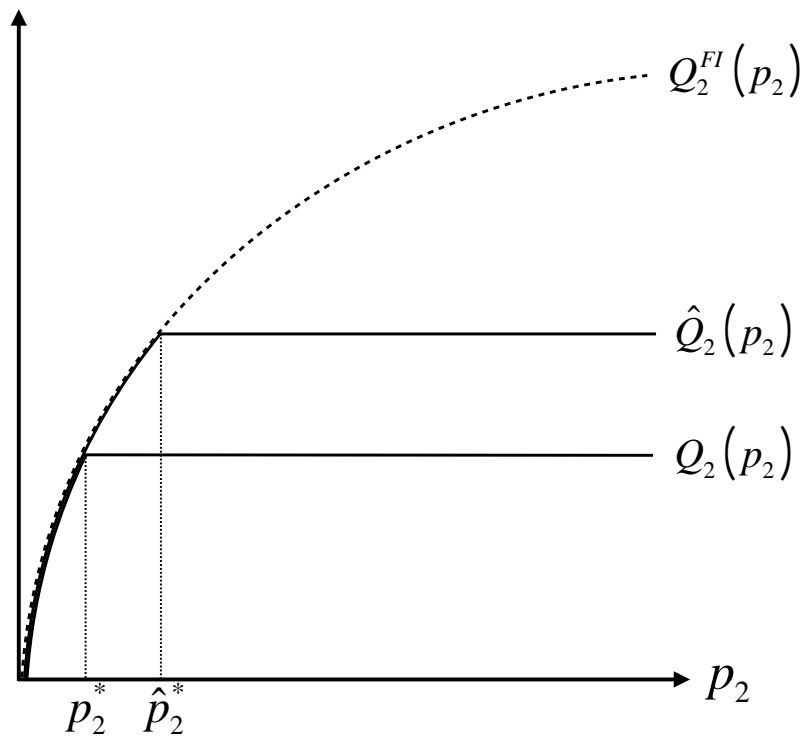

Figure 1: Equilibrium Period-2 Premium Profiles without the Settlement Market under Different Levels of Consumer Overconfidence: $\Delta>\hat{\Delta}$.

Proposition 2 shows that, when the level of policyholders' overconfidence increases from $\hat{\Delta}$ to $\Delta$, a higher degree of reclassification risk insurance (i.e., $p_{2}^{*}<\hat{p}_{2}^{*}$ ) is offered in the second period as Figure 1 depicts. The intuition is as follows. As policyholders become more overconfident, they place more weight on the expected utility from the set of period- 2 contracts and prefer a more actuarially favorable period- 2 contract terms. To maximize the perceived expected utility of the policyholders, the life insurance firms respond by lowering the period-2 premiums and providing a higher degree of reclassification risk in the second period, which implies the insurance firms will suffer a greater loss in period 2. This loss is compensated by a more front-loading contract in the first period in equilibrium: first period premium is higher, and first period death benefit is lower, under $\Delta$ than under $\hat{\Delta}$, namely, $F_{1}<\hat{F}_{1}$ and $Q_{1}>\hat{Q}_{1}$. This argument is reminiscent of Gottlieb and Smetters (2014): higher overconfidence leads to more front-loading in the equilibrium life insurance contract.

The issue, however, is that the policyholders' experienced utility from the equilibrium contract is not the same as the perceived expected utility when they make their life insurance purchase decisions. Following the literature, we use the consumer's experienced utility with the objective probability of losing bequest motives to evaluate the consumer welfare; that is, if a consumer, who has an objective probability of $q$ of losing bequest motives in the second period, purchases a generic long-term contract $C \equiv\left\langle\left(Q_{1}, F_{1}\right),\left(Q_{2}\left(p_{2}\right), F_{2}\left(p_{2}\right)\right): p_{2} \in[0,1]\right\rangle$, then his welfare from the contract is:

$$
\begin{aligned}
W: & =\left[u\left(y-g-Q_{1}\right)+p_{1} v\left(F_{1}\right)\right] \\
& \left.+\left(1-p_{1}\right) \int_{0}^{1}\left\{(1-q)\left[u\left(y+g-Q_{2}\left(p_{2}\right)\right)\right)+p_{2} v\left(F_{2}\left(p_{2}\right)\right)\right]+q u(y+g)\right\} d \Phi\left(p_{2}\right) .
\end{aligned}
$$


Note that in expression (17), the objective probability $q$ enters the calculation, while in the objective function (3) in the optimization problem of the competitive insurers, it is $\tilde{q}$ that enters the calculations. Now we can define the consumer's equilibrium welfare from market environment $(q, \Delta)$ simply as $W$ evaluated at the equilibrium contract $\left\langle\left(Q_{1}(q, \Delta), F_{1}(q, \Delta)\right),\left(Q_{2}\left(p_{2} ; q, \Delta\right), F_{2}\left(p_{2} ; q, \Delta\right)\right): p_{2} \in\right.$ $[0,1]\rangle$ that solves Problem (3):14

Definition 1 The consumer equilibrium welfare in the absence of settlement market, $W(q, \Delta)$, is defined by:

$$
\begin{aligned}
W(q, \Delta) & :=\left[u\left(y-g-Q_{1}(q, \Delta)\right)+p_{1} v\left(F_{1}(q, \Delta)\right)\right] \\
& \left.+\left(1-p_{1}\right) \int_{0}^{1}\left\{(1-q)\left[u\left(y+g-Q_{2}\left(p_{2} ; q, \Delta\right)\right)\right)+p_{2} v\left(F_{2}\left(p_{2} ; q, \Delta\right)\right)\right]+q u(y+g)\right\} d \Phi\left(p_{2}\right) .
\end{aligned}
$$

Since expression (17) coincides with the objective function (3) in the optimization problem of the competitive insurers, and the feasible set does not depend on $\Delta$ as pointed out in Remark 3. when consumers are unbiased, i.e. when $\Delta=0$, it immediately follows that the contract that maximizes (17) and the equilibrium contracts proposed by the life insurance firms coincide. More generally, the following result shows that higher overconfidence leads to lower equilibrium consumer welfare.

\section{Proposition 3 (Higher Overconfidence Leads to Lower Equilibrium Consumer Welfare.)} Fix $q \in(0,1)$. $W(q, \Delta)$ is weakly decreasing in $\Delta$.

When consumers are more overconfident, they are more biased in their belief about the probability of losing bequest motive. As stated in Proposition 2, competitive insurers will cater to the consumers' more biased beliefs by offering more front-loading contracts (higher first period premium, lower first period coverage) in exchange for the more risk reclassification insurance in period 2 which is more subjectively valued if consumers have more biased beliefs. Such catering is effective to attract the consumers in the first period, but the consumers will come to regret, albeit too late, that the risk reclassification insurance offered in the long-term contract is not as valuable as he initially thought, once he observes that his objective probability of losing bequest motive $q$ is higher than what he initially thought. A higher behavioral bias leads to more deviation from the socially optimal contract. Thus, consumer welfare is decreasing in the level of policyholders' overconfidence.

Notice that in the baseline model, when the consumers realize that they have purchased too much reclassification insurance in the second period, it is too late! They realize that they have paid too high a first period premium for the period-2 risk reclassification risk insurance they no longer value as high, but they do not have any recourses. In the next section, we argue that the life settlement market can precisely serve as a recourse for the consumers in the second period when they realize the mistakes due to their overconfidence, and this very presence of the recourse will provide a discipline on the competitive insurers in their equilibrium contract.

\footnotetext{
${ }^{14}$ Here we highlight the fact that the equilibrium contract depends on $(q, \Delta)$ in our notation.
} 


\section{Introducing the Life Settlement Market}

We now introduce the life settlement market at the beginning of period 2 to the baseline model. After the policyholder learns of his period-2 health status $p_{2}$, and whether his bequest motive remains, he has the option to sell the contracts to the settlement firms prior to the resolution of his mortality risk. If the policyholder loses his bequest motive in period 2 , he now has a better option than just lapsing his contract: he can sell his contract to the settlement market, and receive a fraction $\beta \in[0,1)$ of the actuarial value of the contract. The actuarial value of a life insurance contract is the difference between the expected death benefit from the contract and the premium; specifically, in health state $p_{2}$, the actuarial value of the contract is simply $p_{2} F_{2}\left(p_{2}\right)-Q_{2}\left(p_{2}\right)$. If a policyholder decides to sell his insurance to the settlement firm, the settlement firm will continue to pay the second period premiums $Q_{2}\left(p_{2}\right)$ for policyholders, and in return, the life settlement firm becomes the beneficiary of the policy and collect its death benefits if the policyholder die at the end of period 2.

\subsection{Equilibrium Contracts with the Settlement Market}

The presence of the settlement market introduces two main changes to the primary insurers' problem. The first change is on the consumer's expected utility function: the consumer now expects to receive a fraction $\beta$ of the actuarial value of the contract by selling it to the settlement firm in the event he loses bequest motive. The second change is on the insurer's zero-profit condition: because a policyholder without bequest motive in the second period will always sell the policy to the settlement firm instead of lapsing it, the life insurance firm will always have to pay the death benefits. We will show below that these changes will fundamentally alter the way insurance firms provide the long-term insurance contracts in equilibrium.

The equilibrium long-term contract in the presence of the life settlement market, which we denote by $\left\langle\left(Q_{1 s}, F_{1 s}\right),\left(Q_{2 s}\left(p_{2}\right), F_{2 s}\left(p_{2}\right)\right): p_{2} \in[0,1]\right\rangle$, where we use subscript $s$ to indicate "settlement," solves:

$$
\begin{array}{ll}
\max & {\left[u\left(y-g-Q_{1 s}\right)+p_{1} v\left(F_{1 s}\right)\right]} \\
& +\left(1-p_{1}\right) \int_{0}^{1}\left\{(1-\tilde{q})\left[\begin{array}{c}
u\left(y+g-Q_{2 s}\left(p_{2}\right)\right) \\
+p_{2} v\left(F_{2 s}\left(p_{2}\right)\right)
\end{array}\right]+\tilde{q} u\left(y+g+\beta V_{2 s}\left(p_{2}\right)\right)\right\} d \Phi\left(p_{2}\right) \\
\text { s.t. } & \left(Q_{1 s}-p_{1} F_{1 s}\right)+\left(1-p_{1}\right) \int_{0}^{1}\left[Q_{2 s}\left(p_{2}\right)-p_{2} F_{2 s}\left(p_{2}\right)\right] d \Phi\left(p_{2}\right)=0, \\
& Q_{2 s}\left(p_{2}\right)-p_{2} F_{2 s}\left(p_{2}\right) \leq 0 \text { for all } p_{2} \in[0,1], \\
& Q_{2 s}\left(p_{2}\right) \geq 0 \text { for all } p_{2} \in[0,1],
\end{array}
$$

where

$$
V_{2 s}\left(p_{2}\right) \equiv p_{2} F_{2 s}\left(p_{2}\right)-Q_{2 s}\left(p_{2}\right)
$$

is the actuarial value of the period-2 contract with health status $p_{2}$. Note that from the no-lapsation 
condition $(21), V_{2 s}\left(p_{2}\right)$ is always non-negative.

Remark 4 Note that in Problem (19), the objective probability of losing bequest motives, q, enters into neither the zero-profit condition (20) nor the first order conditions (24). Hence, fixing $\tilde{q}$, the set of equilibrium contracts is independent of $q$. This is because life insurance firms have to pay the face amount when policyholders die in period 2 no matter they lose bequest motives or not. Therefore the life insurance firms do not take into account the actual probability of losing bequest motive when they maximize policyholders' perceived utility.

Let $\mu_{s}, \lambda_{s}\left(p_{2}\right)$ and $\gamma_{s}\left(p_{2}\right)$ denote the Lagrange multipliers for constraint (20), 21) and 22) respectively, the first order conditions for Problem 19$)$ with respect to $Q_{1 s}, F_{1 s}, Q_{2 s}\left(p_{2}\right) F_{2 s}\left(p_{2}\right)$ are:

$$
\begin{gathered}
u^{\prime}\left(y-g-Q_{1 s}\right)=\mu_{s}, \\
v^{\prime}\left(F_{1 s}\right)=\mu_{s}, \\
(1-\tilde{q}) u^{\prime}\left(y+g-Q_{2 s}\left(p_{2}\right)\right)+\beta \tilde{q} u^{\prime}\left(y+g+\beta V_{2 s}\left(p_{2}\right)\right)=\mu_{s}+\frac{\lambda_{s}\left(p_{2}\right)+\gamma_{s}\left(p_{2}\right)}{\left(1-p_{1}\right) \phi_{s}\left(p_{2}\right)}, \\
(1-\tilde{q}) v^{\prime}\left(F_{2 s}\left(p_{2}\right)\right)+\beta \tilde{q} u^{\prime}\left(y+g+\beta V_{2 s}\left(p_{2}\right)\right)=\mu_{s}+\frac{\lambda_{s}\left(p_{2}\right)}{\left(1-p_{1}\right) \phi_{s}\left(p_{2}\right)} .
\end{gathered}
$$

Note that the second term $\beta \tilde{q} u^{\prime}\left(y+g+\beta V_{2 s}\left(p_{2}\right)\right)$ in the left hand sides of (24c) and (24d) results from the cash payment the policyholder receives from the settlement firm.

Our first result shows that the presence of life settlement market has a potential disciplinary effect on how much the life insurers may exploit policyholders' overconfidence:

Lemma 5 For all $(q, \Delta) \in[0,1) \times[0,1]$, the solution to Problem (19) must satisfy that $Q_{2 s}\left(p_{2}\right)>0$ for all $p_{2} \in(0,1]$.

Lemma 5 states that the non-negativity condition of $Q_{2 s}\left(p_{2}\right)$ never binds. Recall from Lemma 4 that, when there is no settlement market, it is possible that the life insurers will offer contracts with zero period-2 premiums in equilibrium to maximally exploit the wedge for the overconfident consumers between their subjective value of period-2 reclassification insurance and the objective cost to the insurers to offer such insurance, when $q$ and $\Delta$ are sufficiently large. However, such a pricing strategy will not emerge in equilibrium in the presence of the settlement market. The reason can be seen from (20): when there is a settlement market, the objective cost of providing zero-premium period-2 contracts does not decrease in $q$; in fact, it is independent of $q$ ! Hence, the wedge does not increase with $q$. The presence of the settlement market can be seen as providing a disciplinary effect on the extent to which the life insurers can exploit overconfident consumers: if they are too aggressive in offering risk reclassification insurance and charging highly front-loaded the premiums, they will lose money in the second period in paying the death benefits to the settlement firms.

Lemma 5 implies immediately that $\gamma\left(p_{2}\right)=0$ for all $p_{2} \in(0,1]$. Thus the first order conditions (24) imply that in equilibrium $\left\langle\left(Q_{1 s}, F_{1 s}\right),\left(Q_{2 s}\left(p_{2}\right), F_{2 s}\left(p_{2}\right)\right): p_{2} \in[0,1]\right\rangle$ must satisfy the following 
full-event insurance conditions:

$$
\begin{aligned}
& u^{\prime}\left(y-g-Q_{1 s}\right)=v^{\prime}\left(F_{1 s}\right) \\
& u^{\prime}\left(y+g-Q_{2 s}\left(p_{2}\right)\right)=v^{\prime}\left(F_{2 s}\left(p_{2}\right)\right) \text { for all } p_{2} \in(0,1]
\end{aligned}
$$

that is, all policyholders will obtain full-event insurance in both period 1 and all health states in period 2 when there is settlement market.

Lemma 5 also implies that the characterization of the equilibrium contracts in our setting is identical to that in Fang and Kung (2010) once we replace the variable $q$ in Fang and Kung (2010) with $\tilde{q} 15$ We can partition the period-2 health states into two subsets $\mathcal{B}_{s}$ and $\mathcal{N B}_{s}$ depending on whether the no-lapsation constraint $(21)$ binds. The following lemma is identical to Lemma 3 in Fang and Kung (2010):

Lemma 6 If $p_{2} \in \mathcal{B}_{s}$ and $p_{2}^{\prime} \in \mathcal{N B}_{s}$, then $p_{2}<p_{2}^{\prime}$ and $Q_{2}\left(p_{2}\right)<Q_{2}\left(p_{2}^{\prime}\right)$.

Lemma 6 implies that there is a threshold $p_{2 s}^{*}$ such that $p_{2} \in \mathcal{B}_{s}$ if $p_{2}<p_{2 s}^{*}$ and $p_{2} \in \mathcal{N B}_{s}$ if $p_{2}>p_{2 s}^{*}$. If $p_{2 s}^{*}=1$, then it is obvious that the equilibrium period- 2 contracts is the set of spot contracts. The following lemma characterizes the period-2 premiums $Q_{2 s}\left(p_{2}\right)$ as a function of $p_{2 s}^{*}$ if $p_{2 s}^{*} \in(0,1)$.

Lemma 7 If $p_{2 s}^{*} \in(0,1)$, the equilibrium period 2 premiums $Q_{2 s}\left(p_{2}\right)$ satisfy:

1. for $p_{2} \leq p_{2 s}^{*}, Q_{2 s}\left(p_{2}\right)=Q_{2}^{F I}\left(p_{2}\right)$;

2. for $p_{2}>p_{2 s}^{*}, Q_{2 s}\left(p_{2}\right)$ solves:

$$
\begin{aligned}
& (1-\tilde{q}) u^{\prime}\left(y+g-Q_{2 s}\left(p_{2}\right)\right)+\beta \tilde{q} u^{\prime}\left(y+g+\beta V_{2 s}\left(p_{2}\right)\right) \\
& =(1-\tilde{q}) u^{\prime}\left(y+g-Q_{2}^{F I}\left(p_{2 s}^{*}\right)\right)+\beta \tilde{q} u^{\prime}(y+g) .
\end{aligned}
$$

By Lemma 7, the set of period-2 contracts is fully characterized by $p_{2 s}^{*}$ alone. Moreover, it can be proved from (27) that both $Q_{2 s}\left(p_{2}\right)$ and $V_{2 s}\left(p_{2}\right)$ are strictly increasing in $p_{2}{ }^{16}$ From the first order conditions (24a), 24c) and Lemma 7) the period-1 premium $Q_{1 s}$ is the solution to:

$$
u^{\prime}\left(y-g-Q_{1 s}\right)=(1-\tilde{q}) u^{\prime}\left(y+g-Q_{2}^{F I}\left(p_{2 s}^{*}\right)\right)+\beta \tilde{q} u^{\prime}(y+g) .
$$

To characterize the equilibrium insurance contract, it remains to pin down $p_{2 s}^{*}$, which is determined by the zero-profit condition 20 . The following proposition summarizes the above discussions.

Proposition 4 (Equilibrium Contracts with Settlement Market) The set of equilibrium contracts satisfies the following properties:

\footnotetext{
${ }^{15}$ Recall that Fang and Kung $(2010)$ analyze the case in which consumers have rational beliefs regarding their probability of losing bequest motives, i.e., $\tilde{q}=q$.

${ }^{16}$ See the proof of Proposition 3 in Fang and Kung $(2010)$.
} 


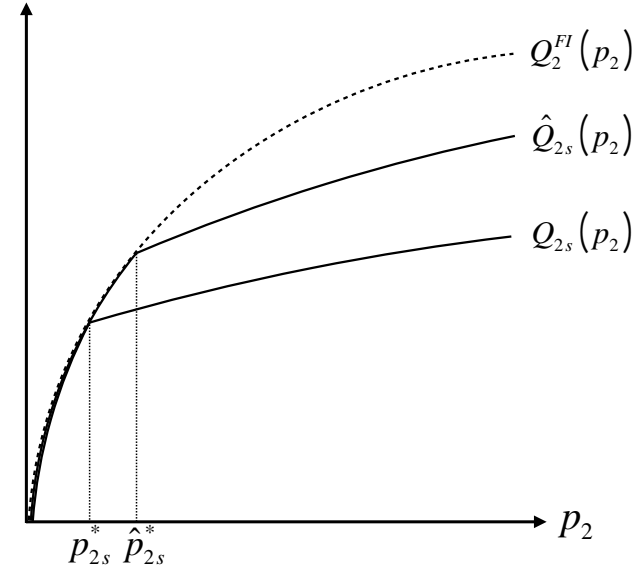

(a) Case I: $\hat{p}_{2 s}^{*}>p_{2 s}^{*}$

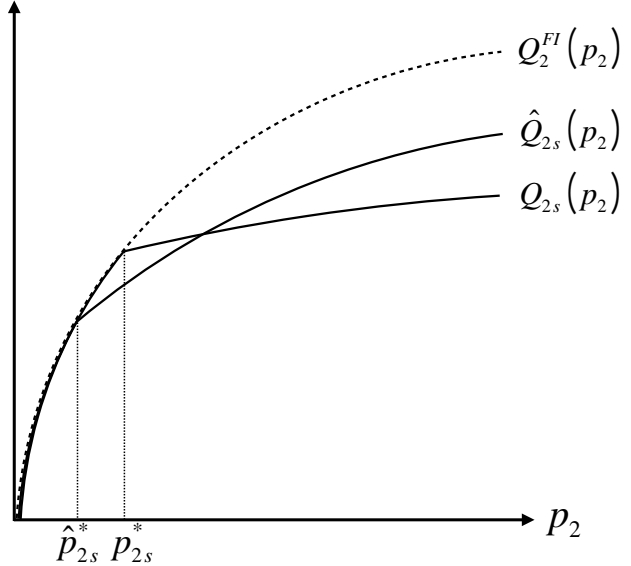

(b) Case II: $\hat{p}_{2 s}^{*}<p_{2 s}^{*}$

Figure 2: Equilibrium Period-2 Premium Profiles with the Settlement Market under Different Levels of Consumer Overconfidence: $\Delta>\hat{\Delta}$.

1. All policyholders receive full-event insurance in period 1 and 2 as defined by (25) and (26).

2. There exists a threshold $p_{2 s}^{*} \in(0,1]$ such that $p_{2} \in \mathcal{B}_{s}$ if $p_{2}<p_{2 s}^{*}$ and $p_{2} \in \mathcal{N B}_{s}$ if $p_{2}>p_{2 s}^{*}$.

3. (a) If $p_{2 s}^{*}<1$, the set of equilibrium contracts is determined by (20), (25), (26) and Lemma 7. Moreover, $Q_{2 s}\left(p_{2}\right)$ and $V_{2 s}\left(p_{2}\right)$ are strictly increasing in $p_{2}$ if $\beta>0$;

(b) If $p_{2 s}^{*}=1$, the equilibrium contract coincides with spot contracts.

In the presence of the settlement market, the life insurance firms no long provide flat premiums in period 2. Instead, they provide partial insurance against reclassification risk in equilibrium. The set of period-2 equilibrium contract is in the form of premium discounts relative to the spot market contracts. Policyholders with higher mortality risk are charged higher premiums, but the equilibrium contract is still in favor of higher $p_{2}$ in the sense the that policyholders with higher $p_{2}$ are offered contracts with higher actuarial values. These insights are identical to those in Fang and Kung (2010).

Proposition 5 Suppose $\hat{\Delta}<\Delta$. Let $\left(\hat{F}_{1 s}, \hat{Q}_{1 s}\right)$ and $\left(F_{1 s}, Q_{1 s}\right)$ be the equilibrium contract in period 1 with $\hat{\Delta}$ and $\Delta$ respectively. If $p_{2 s}^{*}<1$, then $\hat{F}_{1 s}>F_{1 s}$ and $\hat{Q}_{1 s}<Q_{1 s}$.

When consumers become more overconfident, the life insurance firms respond by offering a set of contracts with a higher degree of front-loading (i.e. a higher premium and lower face value) in the first period. The intuition is similar to that of Proposition 2. When policyholders become more overconfident, they demand actuarially more favorable contracts in the second period. As a result, the insurance firms suffer greater losses than before. Therefore, the first period premium increases in equilibrium so as to satisfy the zero-profit condition.

Different from Proposition 2, we can no longer obtain clean comparative statics on the degree of reclassification risk (i.e., $p_{2 s}^{*}$ ) with respect to the degree of consumer overconfidence. Figure 2 depicts 
two possibilities of the change of the second period premiums in equilibrium as the policyholders' overconfidence increases from $\hat{\Delta}$ to $\Delta$ where $\hat{p}_{2 s}^{*}>p_{2 s}^{*}$ holds in Figure 2(a) and $\hat{p}_{2 s}^{*}<p_{2 s}^{*}$ holds in Figure 2(b) ${ }^{17}$ When the secondary life settlement market is present, insurance firms provide contracts with premium discounts rather than flat premiums, whose shape depends on not only $p_{2 s}^{*}$ but also $u(\cdot)$ and $v(\cdot)$ [see Eq. (27)]. Intuitively, the possibility to cash out a positive fraction of the actuarial value of the period-2 contract changes the marginal utility of the policyholders when they lose bequest motives, and hence the shape of the equilibrium premiums changes accordingly.

Analogous to the experienced utility $W$ we defined in (17) for the case when there is no settlement market, we can define the consumer's experienced utility with the objective probability $q$ of losing bequest motives in the second period from a generic long-term contract $C_{s} \equiv$ $\left\langle\left(Q_{1 s}, F_{1 s}\right),\left(Q_{2 s}\left(p_{2}\right), F_{2 s}\left(p_{2}\right)\right): p_{2} \in[0,1]\right\rangle$ as:

$$
\begin{aligned}
W_{s}: & =\left[u\left(y-g-Q_{1 s}\right)+p_{1} v\left(F_{1 s}\right)\right] \\
& +\left(1-p_{1}\right) \int_{0}^{1}\left\{\begin{array}{c}
\left.(1-q)\left[u\left(y+g-Q_{2 s}\left(p_{2}\right)\right)\right)+p_{2} v\left(F_{2 s}\left(p_{2 s}\right)\right)\right] \\
+q u\left(y+g+\beta V_{2 s}\left(p_{2}\right)\right)
\end{array}\right\} d \Phi\left(p_{2}\right) .
\end{aligned}
$$

Note that the expression 290 is very similar to $(17)$ except for the term $\beta V_{2 s}\left(p_{2}\right)$ - the cash amount a policyholder obtains from the settlement firm. The consumer's equilibrium welfare from market environment $(q, \Delta)$ with settlement market is simply $W_{s}$ evaluated at the equilibrium contract $\left\langle\left(Q_{1 s}(q, \Delta), F_{1 s}(q, \Delta)\right),\left(Q_{2 s}\left(p_{2} ; q, \Delta\right), F_{2 s}\left(p_{2} ; q, \Delta\right)\right): p_{2} \in[0,1]\right\rangle$ that solves Problem 19) 18

Definition 2 The consumer equilibrium welfare in the presence of settlement market, $W_{s}(q, \Delta)$, is defined by:

$$
\begin{aligned}
W_{s}(q, \Delta) & :=\left[u\left(y-g-Q_{1 s}(q, \Delta)\right)+p_{1} v\left(F_{1 s}(q, \Delta)\right)\right] \\
& +\left(1-p_{1}\right) \int_{0}^{1}\left\{\begin{array}{c}
\left.(1-q)\left[u\left(y+g-Q_{2 s}\left(p_{2} ; q, \Delta\right)\right)\right)+p_{2} v\left(F_{2 s}\left(p_{2} ; q, \Delta\right)\right)\right] \\
+q u\left(y+g+\beta V_{2 s}\left(p_{2} ; q, \Delta\right)\right)
\end{array}\right\} d \Phi\left(p_{2}\right) .
\end{aligned}
$$

Proposition 6 (Welfare Implication of Overconfidence) Fix $q \in(0,1)$. $W_{s}(q, \Delta)$ is weakly decreasing in $\Delta$.

Proposition 6 establishes a similar comparative statics of consumer welfare with respect to the level of policyholders' overconfidence. The intuition is similar to Proposition 3 . Thus, overconfidence reduces consumer welfare regardless of whether or not the settlement market is present.

\footnotetext{
${ }^{17}$ Assuming that $u(c)=v(c)=c^{0.9},\left(y, g, q, p_{1}\right)=(0.999,0.001,0.8,0.01)$, and $p_{2} \sim \mathcal{U}[0,1]$, the shape of the equilibrium period-2 premiums in Figure 2(a) can be obtained with $\hat{\Delta}=0.05$ and $\Delta=0.5$; and the shape of the equilibrium period-2 premiums in Figure $2(\mathrm{~b})$ can be obtained with $\hat{\Delta}=0.65$ and $\Delta=0.9$.

${ }^{18}$ Here we again highlight the fact that the equilibrium contract depends on $(q, \Delta)$ in our notation.
} 


\section{Welfare Comparison}

In this section, we present our main result on the equilibrium effect of the settlement market on consumer welfare when consumers are overconfident about their bequest motives. It is useful for us to state a result for the case when consumers have correct beliefs regarding the probability that they may lose bequest motive, i.e., when $\tilde{q}=q$ or $\Delta=0$. Proposition 7 in Fang and Kung (2010) considers this case and shows that when the policyholder holds correct belief about the probability of losing his bequest motive, introducing the life settlement market weakly reduces consumer welfare in equilibrium 19 Using the notation for equilibrium welfare $W(\cdot)$ and $W_{s}(\cdot)$ in Definitions 1 and 2 [see Eq. (18) and (30)], Proposition 7 of Fang and Kung (2010) is:

$$
W(q, 0) \geq W_{s}(q, 0)
$$

This result may be somewhat counter-intuitive. The intuition, as Fang and Kung (2010) explained, is as follows. On the one hand, the settlement market allows policyholders to access the actuarial value in their policies, and thus may be interpreted as contributing to market completeness. On the other hand, the settlement market weakens the consumer's ability to commit to not asking for a return of their front loaded premiums in the event that they lose their bequest motive. This weakening of the consumers' commitment power can be interpreted as contributing further to market incompleteness.

As we emphasized in Section 3, when consumers are overconfident about their loss of bequest motives, life settlement market has additional disciplinary effect on the extent to which the primary life insurers can exploit consumers' irrational beliefs in their contract design. Whether the unambiguous result stated in (31) for the case of consumers with rational beliefs will be overturned will crucially depend on the strength of the additional disciplinary effect of the life settlement market.

Our main result, stated as Proposition 7 below, provides sufficient conditions under which life settlement may improve consumer welfare in equilibrium. It is a limiting result and interestingly, it depends on the curvature - captured by the intertemporal elasticity of substitution (IES), or the inverse of the relative risk aversion - of the function $v(\cdot)$. For the ease of our exposition, we denote the IES of $v(\cdot)$ at $c$ by $\eta(c): 20$

$$
\eta(c)=-\frac{v^{\prime}(c)}{c v^{\prime \prime}(c)} .
$$

Proposition 7 Suppose $\eta(c) \geq \alpha>1$ for all $c>0$. There exists a threshold $\bar{q}$ such that for $q \geq \bar{q}$, $W_{s}(q, \Delta)>W(q, \Delta)$ if $\Delta$ is sufficiently large.

The proof of Proposition 7 is relegated to the Appendix. The intuition is as follows. First, note that Proposition 7 requires that $q$ and $\Delta$ to be sufficiently large. When policyholders are

\footnotetext{
${ }^{19}$ See, also, Claim 3 of Proposition 2 in Daily et al. (2008).

${ }^{20}$ Bisin et al. (2015) considers the welfare consequence of banning illiquid assets in a political economy model of public debt where voters have self-control problems and attempt to commit using illiquid assets. They show that in equilibrium, government accumulates inefficiently high level of debt to respond to individuals' desire to undo their commitments using illiquid assets. Interestingly, they find that banning illiquid asset is welfare improving if the coefficient of relative risk aversion is greater than 1 .
} 
overconfident, the settlement market has a new role: it allows policyholders to take actions to correct the mistaken contractual choices in period one resulting from their biased beliefs in the second period. In particular, a fraction $q \Delta$ of policyholders no long remain bequest motives in the second period as expected at the time of purchasing insurance policies. When there is no settlement market, they can only lapse the contract and suffer the utility loss caused by their biased beliefs. However, with the settlement market, policyholders can recover part of the actuarial value of their contracts. Importantly, the threat of consumers, those who unexpectedly find themselves without bequest motives, selling to life settlement firms in the second period provides a curb on how aggressive the life insurers can exploit the consumers' overconfidence in their contract design. In order for the settlement market to be welfare increasing, it requires sufficiently large $q$ and $\Delta$, so that the welfare gain from the disciplinary effect can outweigh the welfare loss due to the lower degree of market completeness as highlighted by (31) for the case of consumers with rational beliefs.

Second, notice that Proposition 7 also requires that the IES of $v(\cdot)$ to be greater than one. This turns out to be related to the general idea that the curvature of $v(\cdot)$ is related to how vulnerable a consumer with biased beliefs can be exploited, in terms of welfare, by firms in the competitive market ${ }^{21}$ To explain the intuition most cleanly, let us consider a utility function with constant IES $\rho>0$, i.e. suppose that:

$$
v(c)=\left\{\begin{array}{ccc}
\frac{c^{1-\frac{1}{\rho}}-1}{1-\frac{1}{\rho}} & \text { if } & \rho>0 \text { and } \rho \neq 1 \\
\ln (c) & \text { if } & \rho=1
\end{array}\right.
$$

From Lemma 4, when $q$ and $\Delta$ are sufficiently large, $Q_{2}\left(p_{2}\right)=0$ for all $p_{2}$ in the case without the settlement market. That is, the life insurance company will cater to the overconfidence of the policyholders by offering zero-premiums contracts in the second period in exchange for a high first period premium. Moreover, the first order conditions $(7 \mathrm{~b})$ and $(7 \mathrm{~d})$ imply that the face amounts $F_{2}\left(p_{2}\right)$ and $F_{1}$ must satisfy

$$
\frac{v^{\prime}\left(F_{2}\left(p_{2}\right)\right)}{v^{\prime}\left(F_{1}\right)}=\frac{1-q}{1-\tilde{q}} \text { for all } p_{2}
$$

Exploiting the constant IES functional form of $v(\cdot)$ as described in (33), we obtain:

$$
\frac{F_{2}\left(p_{2}\right)}{F_{1}}=\left(\frac{1-\tilde{q}}{1-q}\right)^{\rho}=\left(1+\frac{q}{1-q} \Delta\right)^{\rho} \text {. }
$$

Eq. (34) shows that, when $q$ and $\Delta$ are sufficiently large, the life insurers will be offering long-term life insurance policies with a higher face amount in the second period than in the first period. There are two things to note. First, the value of the death benefits are enjoyed by the policyholder's dependents according to $v(\cdot)$; this explains why the curvature of $v(\cdot)$ plays an important role in Proposition 7. Second, a higher death benefits in the second period is valuable only if the policyholder retains the bequest motive. Since the policyholder is overconfident, a higher ratio of

\footnotetext{
${ }^{21}$ See Fang and $\mathrm{Wu}(2017)$ for a more general exploration of consumer vulnerability in behavioral economics.
} 
$F_{2}\left(p_{2}\right) / F_{1}$ is valuable to the consumer ex ante, but not so valuable ex post. Once the consumer loses the bequest motive in the second period, the promised high death benefit $F_{2}\left(p_{2}\right)$ is actually not costly to the insurer. Of course, the insurers will be charging a higher upfront premium payment $Q_{1}$. This is precisely how the insurer exploits the consumer's biased belief!

Eq. (34) highlights that the distortion that results from consumer overconfidence is larger, the higher is the IES parameter $\rho$. Specifically, when $\rho>1$, the equilibrium consumption growth for the dependent is sensitive to changes in the level of policyholders' overconfidence $\Delta$. In the absence of the settlement market, policyholders will obtain an equilibrium contract which specifies a very low face value and a high premium in the first period in exchange for period- 2 contracts of high actuarial values as they become sufficiently overconfident. Such a contract harms policyholders' objective welfare as defined by (18) because the high actuarial values of the period-2 contracts are not objectively valuable to the policyholder when $q$ and $\Delta$ are sufficiently large. In equilibrium, a large portion (i.e. $q \Delta$ ) of the expected utility promised by the set of the equilibrium contracts in the second period is not realized due to policyholder's misperception of the probability of losing bequest motives. To summarize, policyholders with a high value of IES are more vulnerable from their overconfidence and can potentially benefit more from the presence of the settlement market than those with a low value of IES.

Now, with the presence of the settlement market, the set of equilibrium contracts will not deviate as much from the socially optimal one in terms of the degree of front-loading as policyholders become more overconfident. In fact, we can establish a lower bound of the expected utility for policyholders in the first period. To see this, recall from Lemma 5 that, in the presence of the settlement market, contracts with zero period-2 premiums can not be sustained in equilibrium for all $(q, \Delta) \in[0,1) \times[0,1]{ }^{22}$ From the zero-profit condition $(20)$, this in turn implies that there is an upper bound of the amount of front-loading. Thus the presence of the settlement market protects policyholders from obtaining contracts with too much front-loading in the first period as they become more overconfident. Such protection is more valuable to the vulnerable policyholders with high IES than those with a low value of IES and leads the consumer welfare with settlement market to be greater than that without.

Finally, Proposition 7 depends only on the shape of $v(\cdot)$ but not $u(\cdot)$. This is because Proposition 7 is a limiting result. When consumers become sufficiently overconfident and $q$ is sufficiently large, they lose full-event insurance and obtain zero premiums in the second period by Lemma 4. Specifically, in the absence of the settlement market, consumers always consume $y+g$ in the second period if alive regardless of whether or not they lose bequest motives. Consequently, the trade-off between the expected utility of the first period contract and that derived from period-2 contracts is mainly determined by the face amount rather than premium in the limit. In other words, consumer welfare in the absence of the settlement market is mainly driven by $v(\cdot)$ rather than $u(\cdot)$. More formally, the expected utility in the second period can be simplified as $u(y+g)+$ $(1-q) \int_{0}^{1}\left\{p_{2} v\left(F_{2}\left(p_{2}\right)\right)\right\} d \Phi\left(p_{2}\right)$, where the first component is constant over $q$ and $\Delta$.

\footnotetext{
${ }^{22}$ In addition, Lemma $\mathrm{A} 5$ in the Appendix states that $p_{2 s}^{*}$ must be no less than $p_{1}$.
} 


\subsection{Role of IES of $u(\cdot)$ and $v(\cdot)$}

For moderate levels of $q$ and $\Delta$, the curvatures of both $u(\cdot)$ and $v(\cdot)$ should matter in whether the life settlement market can improve consumer welfare in equilibrium. In this subsection, we provide some numerical results. We assume that $(y, g, \beta)=(1,0,0), p_{1}=0.1$, and $p_{2} \sim \mathcal{U}[0,1]{ }^{23}$ Consumers are assumed to exhibit constant IES of $\rho_{1}>0$ and $\rho_{2}>0$ for $u(\cdot)$ and $v(\cdot)$ respectively:

$$
u(c)=\left\{\begin{array}{ccc}
\frac{c^{1-\frac{1}{\rho_{1}}}-1}{1-\frac{1}{\rho_{1}}} & \text { if } & \rho_{1}>0 \text { and } \rho_{1} \neq 1 \\
\ln (c) & \text { if } & \rho_{1}=0 ;
\end{array} \quad v(c)=\left\{\begin{array}{ccc}
\frac{c^{1-\frac{1}{\rho_{2}}}-1}{1-\frac{1}{\rho_{2}}} & \text { if } & \rho_{2}>0 \text { and } \rho_{2} \neq 1 \\
\ln (c) & \text { if } & \rho_{2}=0 .
\end{array}\right.\right.
$$

Figure 3 graphically illustrates our numerical results. The dashed curve is the combination of $\left(\rho_{1}, \rho_{2}\right)$ for which the consumer welfare with the presence of the settlement market is equal to that without for $q=0.4$ and $\Delta=0.3{ }^{24}$ The region of $\left(\rho_{1}, \rho_{2}\right)$ to the right (to the left, respectively) of the dashed curve depicts the combination of $\left(\rho_{1}, \rho_{2}\right)$ for which introducing the settlement is welfare enhancing (welfare reducing, respectively) under equilibrium contract. Similarly, the solid curve represents the contour plot for $q=0.4$ and $\Delta=0.5$; and the dotted curve represents the contour plot for $q=0.9$ and $\Delta=0.9$.

The first pattern to notice is that the contour plot is downward sloping. In words, fixing $\rho_{1}$ ( $\rho_{2}$, respectively), the presence of the settlement increases consumer welfare as $\rho_{2}$ ( $\rho_{1}$, respectively) becomes sufficiently large. Because policyholder's expected utility in each period depends on both $u(\cdot)$ and $v(\cdot)$, the IES of $u(\cdot)$ and $v(\cdot)$ each will have an impact on the IES of policyholder's expected utility. This result confirms the intuition that consumers with a higher IES of either $u(\cdot)$ or $v(\cdot)$ are more vulnerable from their overconfidence, and hence can benefit more from the presence of the settlement market. Second, the solid curve lies below the dashed curve. Therefore, fixing $q$, consumer welfare is more likely to be greater with the presence of the settlement than that without as $\Delta$ increases. This is because the beneficial effect of the settlement increases as $\Delta$ increases. Last, the dotted curve is flatter than the dashed curve in the $\left(\rho_{1}, \rho_{2}\right)$ space, suggesting that the welfare comparison relies more on the IES of $v(\cdot)$ relative to that of $u(\cdot)$ as $q$ and $\Delta$ increase. This confirms the results in Proposition 7 .

\section{Overconfidence of Future Mortality Risk}

In this section, we investigate the influences of consumer's health-related bias on the equilibrium contract and consumer welfare, and demonstrate the robustness of the welfare comparison derived in Proposition 7 for the case of biased belief regarding bequest motive.

In order to simplify the modeling for overconfidence regarding future mortality risks, we simplify the assumption on the distribution of the period-2 mortality risks. In this section, we assume

\footnotetext{
${ }^{23}$ Assuming $\beta=0$ implies instantly that consumers receive no actuarial value of the contract when they lose bequest motive. Therefore, the existence of the settlement market solely serves as a threat to the primary insurance market. Moreover, when $\beta=0$, the equilibrium contract with the presence of the settlement market are flat.

${ }^{24}$ The contour plots are shown only for $\left(\rho_{1}, \rho_{2}\right) \in[0.1,5.1] \times[0.1,5.1]$.
} 


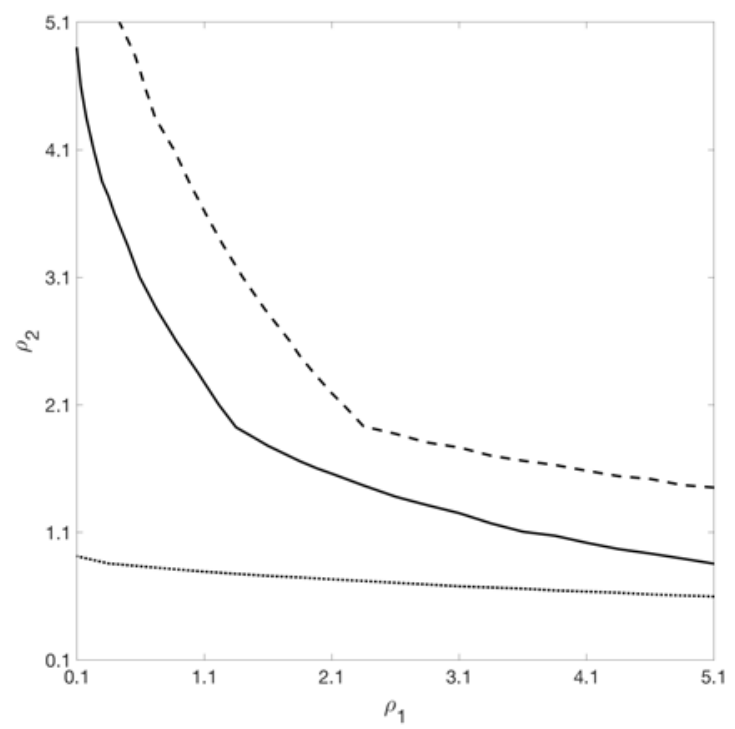

Figure 3: Comparison of Consumer Welfare with and without the Settlement.

Each curve represents combinations of $\left(\rho_{1}, \rho_{2}\right)$ for which the consumer welfare with the presence of the settlement market is equal to that without, for different combinations of $q$ and $\Delta$. To the right of the curve, consumer welfare is higher with life settlement market than without. The dashed curve: $q=0.4$ and $\Delta=0.3$; the solid curve: $q=0.4$ and $\Delta=0.5 ;$ the dotted curve: $q=0.9$ and $\Delta=0.9$.

that the second period mortality risk $p_{2}$ follows a Bernoulli distribution, $p_{2} \in\left\{p_{L}, p_{H}\right\}$, with $p_{H}>p_{L}>p_{1}$, and that the objective distribution is such that

$$
\begin{aligned}
& \operatorname{Pr}\left(p_{2}=p_{L}\right)=\phi_{L} \in(0,1) \\
& \operatorname{Pr}\left(p_{2}=p_{H}\right)=\phi_{H} \equiv 1-\phi_{L} .
\end{aligned}
$$

However, we assume that in period 1, policyholders are overconfident about their future mortality risks, specifically, they believe that $p_{2}$ is drawn from a "better" distribution with

$$
\begin{aligned}
\tilde{\phi}_{L} & =\phi_{L}+\Delta_{m}\left(1-\phi_{L}\right) \\
\tilde{\phi}_{H} & =1-\tilde{\phi}_{L}=\left(1-\Delta_{m}\right)\left(1-\phi_{L}\right),
\end{aligned}
$$

where $\Delta_{m} \in[0,1]$ can be interpreted as the degree of overconfidence of the second period mortality risk.

Equilibrium in the absence of the settlement market. Without the settlement market, the competitive equilibrium contract $\left\langle\left(Q_{1}, F_{1}\right),\left(Q_{2}^{H}, F_{2}^{H}\right),\left(Q_{2}^{L}, F_{2}^{L}\right)\right\rangle$ solves the following maximization 
problem:

$$
\begin{aligned}
& \max \left[u\left(y-g-Q_{1}\right)+p_{1} v\left(F_{1}\right)\right] \\
& \quad+\left(1-p_{1}\right) \sum_{i=L, H} \tilde{\phi}_{i}\left\{(1-q)\left[u\left(y+g-Q_{2}^{i}\right)+p_{i} v\left(F_{2}^{i}\right)\right]+q u(y+g)\right\} \\
& \text { s.t. }\left(Q_{1}-p_{1} F_{1}\right)+\left(1-p_{1}\right)(1-q) \sum_{i=L, H} \phi_{i}\left(Q_{2}^{i}-p_{i} F_{2}^{i}\right)=0, \\
& \quad Q_{2}^{i}-p_{i} F_{2}^{i} \leq 0 \text { for } i \in\{H, L\}, \\
& \quad Q_{2}^{i} \geq 0 \text { for } i \in\{H, L\} .
\end{aligned}
$$

Consumers' overconfidence of future mortality risk introduces an additional concern for the insurers when designing the equilibrium contracts relative to an environment where the consumer bias is with respect to bequest motives. Recall that from the objective function 3 , when consumers underestimate their probability of losing their bequest motives, such bias only distorts the allocation of resources between period 1 and period 2. In contrast, from the objective function (36), overconfidence of future mortality risk distorts the allocation of resources across states in the second period. When policyholders believe that state $p_{L}$ is more likely to occur, the insurer has incentives to decrease the premium in state $p_{L}$ and increase the premium in state $p_{H}$. The following lemma formalizes this intuition.

Lemma 8 There exists a threshold $\bar{\Delta}_{m}<1$ such that $Q_{2}^{H}=Q_{2}^{F I}\left(p_{H}\right)$ if $\Delta_{m}>\bar{\Delta}_{m}$. In addition, $Q_{2}^{L}=0$ if $\phi_{L} \in(0,1)$ is sufficiently small.

From Lemma 8, consumers receive a contract with zero premium in state $p_{L}$ as they become sufficiently overconfident, whereas an actuarially fair full-event insurance is obtained in state $p_{H}$. This contrasts to the result in Lemma 4 where all types receive zero premiums in the second period. The intuition is as follows. As we mentioned, when consumers are overconfident about their bequest motives, this behavioral bias only changes the dynamic trade-off between consumption in the first period and that in the second, and the allocation of consumption across different states in the second period remains optimal. Therefore, insurer has an incentive to make the period- 2 contracts better in all states by decreasing the second period premiums. As a result, as in the rational benchmark model of Hendel and Lizzeri (2003) and Fang and Kung (2012), consumers of a low risk type receive a actuarially fair contract and consumers of a high risk type receive a actuarially favorable contract. However, when consumers are overconfident about the future mortality risk, they put more weight on the low risk state due to their bias. As a result, the insurer has incentives to exploit this bias by making the contract in state $p_{L}$ better in terms of a lower premium, and increasing premiums in state $p_{H}$. Lemma 8 shows that, in the extreme case, the state- $p_{L}$ contract will have zero premium and positive actuarial value, yet, the state- $p_{H}$ contract coincides with the spot contract. Note that consumer bias on mortality risks reverse the predictions of the rational benchmark model of Hendel and Lizzeri (2003) and Fang and Kung (2012): consumers of a high risk type now receive a actuarially fair contract while consumers of a low risk type receive a contract 
with positive actuarial value. As will be discussed in details later, this new feature of the equilibrium contract limits the impact of the settlement market on the shape of the life insurance contract in equilibrium.

Equilibrium with the presence of the settlement market. With the settlement market, the set of equilibrium contracts $\left\langle\left(Q_{1 s}, F_{1 s}\right),\left(Q_{2 s}^{H}, F_{2 s}^{H}\right),\left(Q_{2 s}^{L}, F_{2 s}^{L}\right)\right\rangle$ solves the following maximization problem:

$$
\begin{aligned}
& \max \left[u\left(y-g-Q_{1 s}\right)+p_{1} v\left(F_{1 s}\right)\right] \\
& \quad+\left(1-p_{1}\right) \sum_{i=H, L} \tilde{\phi}_{i}\left\{(1-q)\left[\begin{array}{c}
u\left(y+g-Q_{2 s}^{i}\right) \\
+p_{i} v\left(F_{2 s}^{i}\right)
\end{array}\right]+q u\left(y+g+\beta V_{2 s}^{i}\right)\right\} \\
& \text { s.t. }\left(Q_{1 s}-p_{1} F_{1 s}\right)+\left(1-p_{1}\right) \sum_{i=H, L} \phi_{i}\left[Q_{2 s}^{i}-p_{i} F_{2 s}^{i}\right]=0, \\
& \quad Q_{2 s}^{i}-p_{i} F_{2 s}^{i} \leq 0 \text { for } i \in\{H, L\} \\
& \quad Q_{2 s}^{i} \geq 0 \text { for } i \in\{H, L\} .
\end{aligned}
$$

Again, $V_{2 s}^{i} \equiv p_{i} F_{2 s}^{i}-Q_{2 s}^{i}$ is the actuarial value of the period-2 contract with health status $p_{i}$.

Lemma 9 In the presence of the settlement market, $Q_{2 s}^{H}=Q_{2}^{F I}\left(p_{H}\right), Q_{2 s}^{L}=0$ if $\Delta_{m}$ is sufficiently large and $\phi_{L} \in(0,1)$ is sufficiently small.

Lemma 9 states that, for the case of overconfidence with respect to future mortality rates, the equilibrium pricing pattern for period-2 contracts with the settlement market is identical to that without the settlement market (as in Lemma 8). This is in stark contrast to Lemma 5, which predicts that, for the case of overconfidence with respect to bequest motives, zero premium period2 contracts do not emerge when there is a settlement market while they would in the absence of the settlement market. When consumers exhibit overconfidence of bequest motives, the settlement market protects consumers when they unexpectedly (due to overconfidence) lose bequest motives independent of the second period health status; and the settlement market makes it too costly to the insurer to offer a contract with zero premiums for some states. This force does not apply as strongly when consumers' overconfidence is about the distribution of second period mortality risks. In fact, the equilibrium contract will feature zero premium in state $p_{L}$ as predicted in Lemma 8 but the state $p_{L}$ is much less likely to actually realize than believed by the consumer. Thus the actuarial value in the zero-premium contract for state $p_{L}$ in period 2 is somewhat immune from being exploited and threatened by the settlement market. More explicitly, consider the case where $\phi_{L} \in(0,1)$ is sufficiently small and $\Delta_{m}=1$. In words, consumers subjectively care a lot more about the utility in state $p_{L}$ than they should in period 1 , and in period 2 , they almost always end up with state $p_{H}$. In the absence of life settlement market, the insurer commits to an actuarially fair contract in state $p_{H}$ and a contract with high actuarial value in state $p_{L}$. Different from the case of overconfidence of bequest motive, the promised high actuarial value in state $p_{L}$ will only be cashed 
out by the settlement firm if the consumer actually ends with state $p_{L}$ and loses bequest motive, which occurs with probability $\phi_{L} q$, which is small! As a result, the positive effect of the settlement market to allow biased consumers to correct their "prior mistakes" is not as strong in the case of overconfidence with respect to future mortality risks. This in turn implies that life settlement is limited in its potential to unlock the actuarial value of a contract, and allows for zero-premium contract with large actuarial values in state $p_{L}$ to persist in equilibrium.

Welfare comparison. Lemma 8 and 9 show that the role of the settlement market in changing the equilibrium contract and correcting consumers' mistakes due to their wrong beliefs is limited when consumer overconfidence is about future mortality risks. However, we will show that life settlement still constrains the life insurers' ability to exploit the consumers' biased beliefs in a different manner, and we provide an example in which the presence of the settlement market can again be welfare improving.

As in Section 4, we define the consumers' equilibrium expected welfare using the objective mortality risks:

$$
\begin{aligned}
W^{m}\left(\phi_{L}, \Delta_{m}\right) & :=\left[u\left(y-g-Q_{1}\left(\phi_{L}, \Delta_{m}\right)\right)+p_{1} v\left(F_{1}\left(\phi_{L}, \Delta_{m}\right)\right)\right] \\
+ & \left(1-p_{1}\right) \sum_{i=L, H} \phi_{i}\left\{(1-q)\left[u\left(y+g-Q_{2}^{i}\left(\phi_{L}, \Delta_{m}\right)\right)+p_{i} v\left(F_{2}^{i}\left(\phi_{L}, \Delta_{m}\right)\right)\right]+q u(y+g)\right\} \\
W_{s}^{m}\left(\phi_{L}, \Delta_{m}\right) & :=\left[u\left(y-g-Q_{1 s}\left(\phi_{L}, \Delta_{m}\right)\right)+p_{1} v\left(F_{1 s}\left(\phi_{L}, \Delta_{m}\right)\right)\right] \\
& +\left(1-p_{1}\right) \sum_{i=L, H} \phi_{i}\left\{\begin{array}{c}
(1-q)\left[u\left(y+g-Q_{2 s}^{i}\left(\phi_{L}, \Delta_{m}\right)\right)+p_{i} v\left(F_{2 s}^{i}\left(\phi_{L}, \Delta_{m}\right)\right)\right] \\
+q u\left(y+g+\beta V_{2 s}^{i}\left(\phi_{L}, \Delta_{m}\right)\right)
\end{array}\right\} .
\end{aligned}
$$

Following the discussions in Section 4, we assume that

$$
u(c)=v(c)=\left\{\begin{array}{ccc}
\frac{c^{1-\frac{1}{\rho}}-1}{1-\frac{1}{\rho}} & \text { if } & \rho>0 \text { and } \rho \neq 1 \\
\ln (c) & \text { if } & \rho=1 .
\end{array}\right.
$$

Thus, both utility functions exhibit constant IES of $\rho>0$. To simplify the analysis, we shut down the channel of unlocking the actuarial value of a contract by assuming $\beta=025$

Proposition 8 Suppose $u(\cdot)=v(\cdot)$, and both utility functions exhibit constant IES of $\rho>0$. Moreover, $\beta=0$. Then consumer's welfare is higher (lower, respectively) with the presence of the settlement market than without if $\rho>1\left(\rho<1\right.$, respectively) when $\Delta_{m} \in(0,1)$ is sufficiently large and $\phi_{L} \in(0,1)$ is sufficiently small.

We now provide the intuition for Proposition 8. When $\Delta_{m} \in(0,1)$ is sufficiently large and $\phi_{L} \in(0,1)$ is sufficiently small, the consumers' welfare in the second period is mainly determined by

\footnotetext{
${ }^{25}$ The result is robust when $\beta \in(0,1)$.
} 
the contract offered in state $p_{H}\left(\right.$ since $\phi_{L}$ is small); because Lemmas 8 and 9 show that the state- $p_{H}$ contract terms are identical with or without the settlement market, the comparison of consumer's welfare will mainly hinge on their utility obtained from the first period contract. Not surprisingly, in the absence of life settlement market, the insurer will cater to the consumer overconfidence by offering a favorable contract term in state- $p_{L}$ in period-2 in the form of a higher face amount $F_{2}^{L}$. Such promises of high death benefit $F_{2}^{L}$ is not costly for the insurer because $\phi_{L}$ is low, but will be much valued by the consumer because $\Delta_{m}$ is high. Indeed, it can be shown that, in the absence of the life settlement market, the ratio between $F_{2}^{L}$ and $F_{1}$ is given by:

$$
\frac{F_{2}^{L}}{F_{1}}=\left(\frac{\tilde{\phi}_{L}}{\phi_{L}}\right)^{\rho}=\left(\frac{\phi_{L}+\Delta_{m}\left(1-\phi_{L}\right)}{\phi_{L}}\right)^{\rho} .
$$

The term $\left(\tilde{\phi}_{L} / \phi_{L}\right)^{\rho}>1$ measures the distortion of the contract in equilibrium due to consumer's biased belief. Intuitively, such distortion leads to more welfare loss when the magnitude of overconfidence becomes large (i.e. large $\Delta_{m}$ and small $\phi_{L}$ ), and when consumers have weak propensity towards consumption smoothing (i.e. $\rho>1$ ).

In the presence of life settlement market, the ratio between $F_{2 s}^{L}$ and $F_{1 s}$ will now be more balanced because increasing the state- $p_{L}$ face amount in period 2 can actually be costly to the insurance company (when the consumer loses bequest motive in state- $p_{L}$ ); indeed, we can show that:

$$
\frac{F_{2 s}^{L}}{F_{1 s}}=\left[\frac{\tilde{\phi}_{L}}{\phi_{L}} \times(1-q)\right]^{\rho}
$$

The additional term $(1-q)^{\rho}$ captures the beneficial effect of the settlement market on disciplining the primary life insurance market, which is decreasing in $q$ because the life insurer needs to always pay the face amount due to the presence of the settlement market. This disciplining effect becomes stronger and more important as the welfare loss caused by consumer's overconfidence in the absence of the settlement market is large enough, that is, $\rho>1$. Therefore, introducing a settlement market can be welfare enhancing (reducing, respectively) when $\rho>1$ ( $\rho<1$, respectively).

Note that in Proposition 8 , in order for the life settlement to strictly affect the consumer welfare, $\phi_{L}$ must be positive and small. In the limit when $\Delta_{m} \rightarrow 1$ and $\phi_{L} \rightarrow 0$, it is clear that the contracts with and the without the settlement market will exactly coincide. Thus life settlement does not affect consumer welfare in the limit.

The role of IES of $u(\cdot)$ and $v(\cdot)$. Proposition 8 presents limiting result of $\phi_{L}$ and $\Delta_{m}$, assuming $u(\cdot)=v(\cdot)$. In this subsection we report numerical results to isolate the role of IES of $u(\cdot)$ and $v(\cdot)$ for intermediate values of $q$ and $\Delta_{m}$. To proceed, we assume that $(y, g, \beta)=(1,0,0), q=0.4$, $\left(p_{1}, p_{2}^{L}, p_{2}^{H}\right)=(0.1,0.2,0.8)$, and $\left(\phi_{L}, \phi_{H}\right)=(0.5,0.5)$. Consumers are assumed to exhibit constant IES of $\rho_{1}$ and $\rho_{2}$ for $u(\cdot)$ and $v(\cdot)$ respectively, as in $(35)$.

Figure 4 graphically illustrates our numerical results. The dashed curve is the combination of $\left(\rho_{1}, \rho_{2}\right)$ for which the consumer welfare with the presence of the settlement market is equal to 


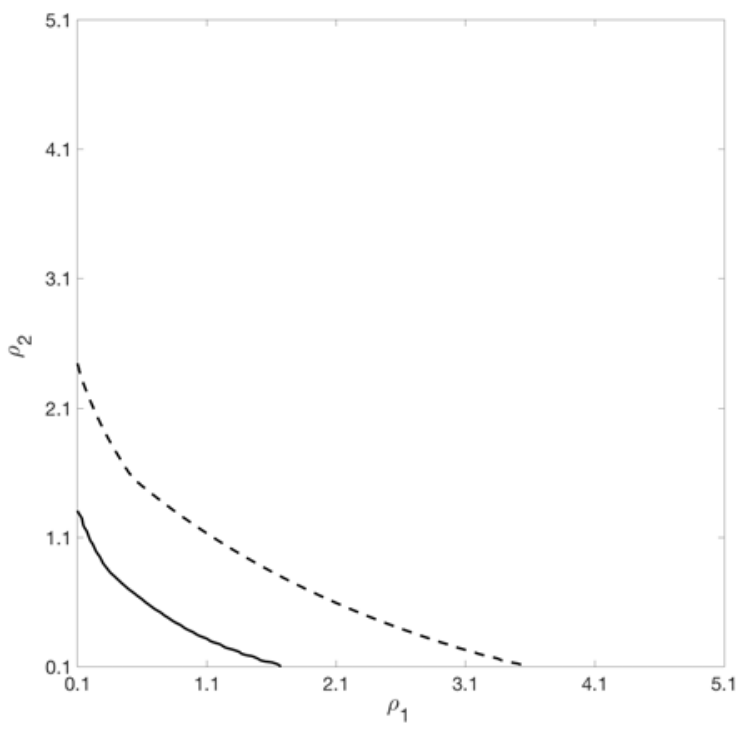

Figure 4: Comparison of Consumer Welfare with and without the Settlement.

Each curve represents combinations of $\left(\rho_{1}, \rho_{2}\right)$ for which the consumer welfare with the presence of the settlement market is equal to that without, for different combinations of $q$ and $\Delta_{m}$. To the right (respectively, left) of the curve, consumer welfare is higher (respectively, lower) with life settlement market than without. The dashed curve: $q=0.4$ and $\Delta_{m}=0.3 ;$ the solid curve: $q=0.4$ and $\Delta_{m}=0.5$.

that without for $\Delta_{m}=0.3{ }^{26}$ The region of $\left(\rho_{1}, \rho_{2}\right)$ to the right (to the left, respectively) of the dashed curve depicts the combination of $\left(\rho_{1}, \rho_{2}\right)$ for which introducing the settlement is welfare enhancing (welfare reducing, respectively) under equilibrium contract. Similarly, the solid curve represents the contour plot for $\Delta_{m}=0.5$. Similar to the result of overconfidence of bequest motive, the contour line is downward sloping in the $\left(\rho_{1}, \rho_{2}\right)$ space, and shifts downwards as the consumer becomes more overconfident.

\section{Conclusion}

In this paper, we analyze how the life settlement market - the secondary market for life insurance - may affect consumer welfare in a dynamic equilibrium model of life insurance with one-sided commitment and overconfident policyholders who may lapse their policies when they lose their bequest motives. We consider two forms of overconfidence: (1) the policyholders may underestimate their probability of losing their bequest motive; (2) the policyholders may be overconfident about their future mortality risks. For the case of overconfidence with respect to bequest motives, we show that in the absence of life settlement overconfident consumers may buy "too much" reclassification risk insurance for later periods in the competitive equilibrium. In contrast, when consumers are overconfident about their future mortality rates in the sense that they put too high a subjective probability on the low-mortality state, the competitive equilibrium contract in the absence of

\footnotetext{
${ }^{26}$ Again, the contour plots are shown only for $\left(\rho_{1}, \rho_{2}\right) \in[0.1,5.1] \times[0.1,5.1]$.
} 
life settlement exploits the consumer bias by offering them very high face amounts in the lowmortality state. In both cases, life settlement market can impose a discipline on the extent to which overconfident consumers can be exploited by the primary insurers. We show that life settlement may increase the equilibrium consumer welfare of overconfident consumers when they are sufficiently vulnerable in the sense that they have a sufficiently large intertemporal elasticity of substitution of consumption.

There are several directions for future research. First, in this paper we studied the role of consumer overconfidence in determining the shape of the equilibrium life insurance contract. It would be interesting to empirically test the existence of policyholders' overconfidence based on the predictions in this paper. Second, we follow Daily et al. (2008) and Fang and Kung (2010), and assume that policyholders may lapse the insurance contract when they lose their bequest motives throughout the paper. It is important to analyze the welfare implications of the settlement market in a unified framework where lapsation is driven by bequest motive shocks as well as negative income shocks. Finally, in our paper we have identified the potential role of IES in the welfare analysis of the life insurance market when policyholders are not fully rational. Another intriguing research avenue would be to generalize the economic insight of IES and consumer vulnerability

to other markets (e.g., the credit market, and the labor market) and quasi-Bayesian models, and investigate the welfare impact and efficacy of different government policies (see Fang and Wu (2017) for some preliminary results).

\section{References}

American Council of Life Insurers, Life Insurers Fact Book 2014, 2014.

Bisin, Alberto, Alessandro Lizzeri, and Leeat Yariv, "Government Policy with Time Inconsistent Voters," American Economic Review, 2015, 105(6), 1711-1737.

Daily, Glenn, Igal Hendel, and Alessandro Lizzeri, "Does the Secondary Life Insurance Market Threaten Dynamic Insurance?," American Economic Review, 2008, 98 (2), 151-156.

de la Rosa, Leonidas Enrique, "Overconfidence and Moral Hazard," Games and Economic Behavior, 2011, 73 (2), 429-451.

Dellavigna, Stephano and Ulrike Malmendier, "Paying Not to Go to The Gym," American Economic Review, 2006, 96, 694-719.

Fang, Hanming and Edward Kung, "How Does Life Settlement Affect the Primary Life Insurance Market?," Working Paper 15761, National Bureau of Economic Research, February 2010.

_ and _ , "Why Do Life Insurance Policyholders Lapse? The Roles of Income, Health and Bequest Motive Shocks," Working Paper 17899, National Bureau of Economic Research, March 2012.

- and Giuseppe Moscarini, "Morale Hazard," Journal of Monetary Economics, 2005, Vol. 52, 749-777. 
_ and Zenan Wu, "Consumer Vulnerability in Behavioral Economics," Work in Progress, 2017.

Gottlieb, Daniel, "Competition over Time-Inconsistent Consumers," Journal of Public Economic Theory, 2008, 10(4), 673-684.

- and Kent Smetters, "Lapse-Based Insurance," Working Paper, 2014.

Grubb, Michael D., "Selling to Overconfident Consumers," American Economic Review, 2009, 99 (5), 1770-1807.

_, "Overconfident Consumers in the Marketplace," Journal of Economic Perspectives, 2015, Vol. 29, No. 4, 9-36.

Hendel, Igal and Alessandro Lizzeri, "The Role of Commitment in Dynamic Contracts: Evidence from Life Insurance," The Quarterly Journal of Economics, 2003, 118 (1), 299-327.

Januário, Afonso V. and Narayan Y. Naik, "Testing for Adverse Selection in Life Settlements: The Secondary Market for Life Insurance Policies," Working Paper, 2014.

Kahneman, Daniel, Peter P. Wakker, and Rakesh Sarin, "Back to Bentham? Explorations of Experienced Utility," Quarterly Journal of Economics, 1997, 112(2), 375-405.

Köszegi, Botond, "Behavioral Contract Theory," Journal of Economic Literature, 2014, 52 (4), $1075-1118$.

Martin, Susan Lorde, "Betting on the Lives of Strangers: Life Settlements, STOLI, and Securitization," University of Pennsylvania Journal of Business Law, 2010, 13 (1), 173-219.

Robb, Kathryn A., Anne Miles, and Jane Wardle, "Subjective and Objective Risk of Colorectal Cancer (UK)," Cancer Causes \& Control, 2004, 15 (1), 21-25.

Rothschild, Michael and Joseph Stiglitz, "Equilibrium in Competitive Insurance Markets: An Essay on the Economics of Imperfect Information," The Quarterly Journal of Economics, 1976, 90 (4), 629-649.

Sandroni, Alvaro and Francesco Squintani, "Overconfidence, Insurance, and Paternalism," The American Economic Review, 2007, 97 (5), 1994-2004.

Santos-Pinto, Luís, "Positive Self-Image and Incentives in Organisations," The Economic Journal, 2008, 118 (531), 1315-1332.

Spinnewijn, Johannes, "Unemployed but Optimistic: Optimal Insurance Design with Biased Beliefs," Journal of the European Economic Association, 2015, 13 (1), 130-167.

Weinstein, Neil D., "Unrealistic Optimism about Future Life Events.," Journal of Personality and Social Psychology, 1980, 39 (5), 806. 


\section{Appendix: Proofs}

\section{Proof of Lemma 1}

Proof. First, we show that $Q_{2}\left(p_{2}\right) \leq Q_{2}\left(p_{2}^{\prime}\right)$. From the complementary slackness condition (8a), $p_{2}^{\prime} \in \mathcal{N B}$ implies instantly that $\lambda\left(p_{2}^{\prime}\right)=0$. Moreover, the Inada condition on $v(\cdot)$ implies that $F_{2}\left(p_{2}\right)>0$. Together with the postulated $p_{2} \in \mathcal{B}$, we must have $Q_{2}\left(p_{2}\right)=p_{2} F_{2}\left(p_{2}\right)>0$, which in turn implies that $\gamma\left(p_{2}\right)=0$ from the complementary slackness condition (8b). Therefore,

$$
\begin{aligned}
(1-\tilde{q}) u^{\prime}\left(y+g-Q_{2}\left(p_{2}\right)\right) & =(1-q) \mu+\frac{\lambda\left(p_{2}\right)+\gamma\left(p_{2}\right)}{\left(1-p_{1}\right) \phi\left(p_{2}\right)} \\
& \leq(1-q) \mu+\frac{\lambda\left(p_{2}^{\prime}\right)+\gamma\left(p_{2}^{\prime}\right)}{\left(1-p_{1}\right) \phi\left(p_{2}^{\prime}\right)}=(1-\tilde{q}) u^{\prime}\left(y+g-Q_{2}\left(p_{2}^{\prime}\right)\right),
\end{aligned}
$$

where the two equalities follow from the first order condition $7 \mathrm{c}$; and the inequality follows from $\lambda\left(p_{2}\right)+\gamma\left(p_{2}\right) \leq 0 \leq \lambda\left(p_{2}^{\prime}\right)+\gamma\left(p_{2}^{\prime}\right)$. From the strict concavity of $u(\cdot)$ and $\tilde{q}<1$, we must have $Q_{2}\left(p_{2}\right) \leq Q_{2}\left(p_{2}^{\prime}\right)$. Similarly, it can be shown that $F_{2}\left(p_{2}\right) \geq F_{2}\left(p_{2}^{\prime}\right)$.

Next, we show that $p_{2}<p_{2}^{\prime}$. Suppose to the contrary that $p_{2} \geq p_{2}^{\prime}$. Then we must have

$$
Q_{2}\left(p_{2}\right) \leq Q_{2}\left(p_{2}^{\prime}\right)<p_{2}^{\prime} F_{2}\left(p_{2}^{\prime}\right) \leq p_{2} F_{2}\left(p_{2}\right)
$$

where the second inequality follows from $p_{2}^{\prime} \in \mathcal{N B}$, and the last inequality follows from the postulated $p_{2} \geq p_{2}^{\prime}$ and $F_{2}\left(p_{2}\right) \geq F_{2}\left(p_{2}^{\prime}\right)$, a contradiction against $p_{2} \in \mathcal{B}$. This completes the proof.

\section{Proof of Lemma 2}

Proof. Suppose to the contrary that there exist two health states $\tilde{p}_{2} \neq 0$ and $\tilde{p}_{2}^{\prime} \neq 0$ such that $Q_{2}\left(\tilde{p}_{2}^{\prime}\right)>0=Q_{2}\left(\tilde{p}_{2}\right)$, then $\gamma\left(\tilde{p}_{2}\right) \geq 0=\gamma\left(\tilde{p}_{2}^{\prime}\right)$ from the complementary slackness condition 8a. Moreover, the Inada condition on $v(\cdot)$ implies that $F_{2}\left(\tilde{p}_{2}\right)>0$. Therefore,

$$
Q_{2}\left(\tilde{p}_{2}\right)-\tilde{p}_{2} F_{2}\left(\tilde{p}_{2}\right)=0-\tilde{p}_{2} F_{2}\left(\tilde{p}_{2}\right)<0
$$

Together with the complementary slackness condition $8 \mathrm{~b}$, we must have $\lambda\left(\tilde{p}_{2}\right)=0 \geq \lambda\left(\tilde{p}_{2}^{\prime}\right)$. Thus,

$$
\begin{aligned}
(1-\tilde{q}) u^{\prime}\left(y+g-Q_{2}\left(\tilde{p}_{2}^{\prime}\right)\right) & =(1-q) \mu+\frac{\lambda\left(\tilde{p}_{2}^{\prime}\right)+\gamma\left(\tilde{p}_{2}^{\prime}\right)}{\left(1-p_{1}\right) \phi\left(\tilde{p}_{2}^{\prime}\right)} \\
& \leq(1-q) \mu+\frac{\lambda\left(\tilde{p}_{2}\right)+\gamma\left(\tilde{p}_{2}\right)}{\left(1-p_{1}\right) \phi\left(\tilde{p}_{2}\right)}=(1-\tilde{q}) u^{\prime}\left(y+g-Q_{2}\left(\tilde{p}_{2}\right)\right),
\end{aligned}
$$

where the two equalities follow from the first order condition $(7 \mathrm{c})$; and the inequality follows from $\lambda\left(\tilde{p}_{2}^{\prime}\right)+\gamma\left(\tilde{p}_{2}^{\prime}\right) \leq 0 \leq \lambda\left(\tilde{p}_{2}\right)+\gamma\left(\tilde{p}_{2}\right)$. Thus, $Q_{2}\left(\tilde{p}_{2}^{\prime}\right) \leq Q_{2}\left(\tilde{p}_{2}\right)$ from the strict concavity of $u(\cdot)$, which is a contradiction to the postulated $Q_{2}\left(\tilde{p}_{2}^{\prime}\right)>0=Q_{2}\left(\tilde{p}_{2}\right)$. This completes the proof.

\section{Proof of Lemma 4}


Proof. Before proving the lemma, it is useful to state several intermediary results.

Lemma A1 If $\Delta=0, Q_{2}\left(p_{2}\right)>0$ for all $p_{2} \in(0,1]$.

Proof. Suppose that there exists a health state $\hat{p}_{2} \in(0,1]$ such that $Q_{2}\left(\hat{p}_{2}\right)=0$. Because $F_{2}\left(p_{2}\right)>0$ for all $p_{2}>0$ from the Inada condition on $v(\cdot)$, we must have $Q_{2}\left(\hat{p}_{2}\right)-\hat{p}_{2} F_{2}\left(\hat{p}_{2}\right)<0$, implying $\lambda\left(\hat{p}_{2}\right)=0$. Combining the first order conditions $(7 \mathrm{a})$ and $(7 \mathrm{c}$ ) yields

$$
u^{\prime}(y+g)=u^{\prime}\left(y+g-Q_{2}\left(\hat{p}_{2}\right)\right)=\frac{1-q}{1-\tilde{q}} \mu+\frac{1}{1-\tilde{q}} \frac{\lambda\left(\hat{p}_{2}\right)+\gamma\left(\hat{p}_{2}\right)}{\left(1-p_{1}\right) \phi\left(\hat{p}_{2}\right)} \geq \frac{1-q}{1-\tilde{q}} \mu=u^{\prime}\left(y-g-Q_{1}\right),
$$

where the inequality follow from $\lambda\left(\hat{p}_{2}\right)=0$ and $\gamma\left(\hat{p}_{2}\right) \geq 0$, and the last equality follows from $\Delta=0$. From Remark 1. $Q_{1} \geq p_{1} F_{1}>0$. Therefore, we must have $y-g-Q_{1}<y+g$ and $u^{\prime}(y+g)<u^{\prime}\left(y-g-Q_{1}\right)$, a contradiction.

Lemma A2 Fix $q$ and $p_{2} \neq 0$. Denote the equilibrium period-2 premium at health state $p_{2}$ with respect to overconfidence $\Delta$ and $\Delta^{\prime}$ by $Q_{2}\left(p_{2}\right)$ and $Q_{2}^{\prime}\left(p_{2}\right)$ respectively. If $Q_{2}\left(p_{2}\right)>0=Q_{2}^{\prime}\left(p_{2}\right)$, then $\Delta<\Delta^{\prime}$.

Proof. For notational convenience, we use the prime symbol to refer to the variables when the degree of consumer overconfidence is $\Delta^{\prime}$. Suppose to the contrary that $\Delta \geq \Delta^{\prime}$, then it follows immediately that $\tilde{q} \leq \tilde{q}^{\prime}$. From Lemma $2, Q_{2}^{\prime}\left(p_{2}\right)=0$ for all $p_{2} \in(0,1]$. Therefore, $\gamma^{\prime}\left(p_{2}\right) \geq 0$ and $\lambda^{\prime}\left(p_{2}\right)=0$ for all $p_{2} \in(0,1]$, which in turn implies that $u^{\prime}\left(y+g-Q_{2}\left(p_{2}\right)\right)=v^{\prime}\left(F_{2}\left(p_{2}\right)\right)$ for all $p_{2} \in(0,1]$. Similarly, $Q_{2}\left(p_{2}\right)>0$ for all $p_{2} \in(0,1]$, implying that $\gamma\left(p_{2}\right)=0$ and $\lambda\left(p_{2}\right) \leq 0$ for all $p_{2} \in(0,1]$.

We first show that the period-2 face value under $\Delta$ is strictly greater than that under $\Delta^{\prime}$ for all $p_{2} \in(0,1]$. Combining the first order conditions $(7 \mathrm{c})$ and $(7 \mathrm{~d})$, we have

$$
\begin{aligned}
v^{\prime}\left(F_{2}^{\prime}\left(p_{2}\right)\right) & =u^{\prime}\left(y+g-Q_{2}^{\prime}\left(p_{2}\right)\right)-\frac{1}{\left(1-\tilde{q}^{\prime}\right)} \times \frac{\gamma^{\prime}\left(p_{2}\right)}{\left(1-p_{1}\right) \phi\left(p_{2}\right)} \\
& <u^{\prime}\left(y+g-Q_{2}\left(p_{2}\right)\right)=v^{\prime}\left(F_{2}\left(p_{2}\right)\right), \text { for all } p_{2} \in(0,1],
\end{aligned}
$$

where the inequality follows from that $\gamma^{\prime}\left(p_{2}\right) \geq 0$ and $Q_{2}\left(p_{2}\right)>0=Q_{2}^{\prime}\left(p_{2}\right)$. Thus, $F_{2}^{\prime}\left(p_{2}\right)>F_{2}\left(p_{2}\right)$ for all $p_{2} \in(0,1]$ from the strict concavity of $v(\cdot)$.

Next, we show that the period- 1 face value under $\Delta$ is strictly greater than that under $\Delta$. Fixing $p_{2} \neq 0$, combining conditions $7 \mathrm{~b}$ and $7 \mathrm{c}$ yields

$$
\begin{aligned}
(1-q) v^{\prime}\left(F_{1}\right) & =(1-\tilde{q}) u^{\prime}\left(y+g-Q_{2}\left(p_{2}\right)\right)-\frac{\lambda\left(p_{2}\right)+\gamma\left(p_{2}\right)}{\left(1-p_{1}\right) \phi\left(p_{2}\right)} \\
& \geq(1-\tilde{q}) u^{\prime}\left(y+g-Q_{2}\left(p_{2}\right)\right) \\
& >\left(1-\tilde{q}^{\prime}\right) u^{\prime}\left(y+g-Q_{2}^{\prime}\left(p_{2}\right)\right) \\
& \geq\left(1-\tilde{q}^{\prime}\right) u^{\prime}\left(y+g-Q_{2}^{\prime}\left(p_{2}\right)\right)-\frac{\lambda^{\prime}\left(p_{2}\right)+\gamma^{\prime}\left(p_{2}\right)}{\left(1-p_{1}\right) \phi\left(p_{2}\right)}=(1-q) v^{\prime}\left(F_{1}^{\prime}\right) .
\end{aligned}
$$


The first inequality follows from $\gamma\left(p_{2}\right)=0$ and $\lambda\left(p_{2}\right) \leq 0$; the second inequality follows from $Q_{2}\left(p_{2}\right)>0=Q_{2}^{\prime}\left(p_{2}\right)$; and the third inequality follows from $\gamma^{\prime}\left(p_{2}\right) \geq 0$ and $\lambda^{\prime}\left(p_{2}\right)=0$. The above inequality implies instantly that $F_{1}^{\prime}>F_{1}$ and $Q_{1}^{\prime}<Q_{1}$.

To complete the proof, notice that the expected profit under $\Delta^{\prime}$ can be bounded from above by,

$$
\begin{aligned}
& \left(Q_{1}^{\prime}-p_{1} F_{1}^{\prime}\right)+\left(1-p_{1}\right)(1-q) \int_{0}^{1}\left[Q_{2}^{\prime}\left(p_{2}\right)-p_{2} F_{2}^{\prime}\left(p_{2}\right)\right] d \Phi\left(p_{2}\right) \\
< & \left(Q_{1}-p_{1} F_{1}\right)+\left(1-p_{1}\right)(1-q) \int_{0}^{1}\left[Q_{2}\left(p_{2}\right)-p_{2} F_{2}\left(p_{2}\right)\right] d \Phi\left(p_{2}\right)=0,
\end{aligned}
$$

which is a contradiction to the zero-profit condition (4) when consumers' overconfidence is $\Delta^{\prime}$.

Lemma A3 Fixing $\tilde{q} \in[0,1)$, there exists $q \in(\tilde{q}, 1)$ such that $Q_{2}\left(p_{2}\right)=0$ for some $p_{2} \neq 0$.

Proof. Suppose to the contrary that there exists $\tilde{q}$ such that $Q_{2}\left(p_{2}\right)>0$ for all $q \in(\tilde{q}, 1)$. This implies instantly that $\lambda\left(p_{2}\right) \leq 0$ and $\gamma\left(p_{2}\right)=0$ for all $p_{2} \in(0,1]$. Then we must have $v^{\prime}\left(F_{2}\left(p_{2}\right)\right)=u^{\prime}\left(y+g-Q_{2}\left(p_{2}\right)\right)>u^{\prime}(y+g)$ for all $p_{2} \in(0,1]$. Therefore, $F_{2}\left(p_{2}\right)$ is bounded from above by $v^{\prime-1}\left(u^{\prime}(y+g)\right)$; and the period-1 expected profit is bounded from above by,

$$
\begin{aligned}
Q_{1}-p_{1} F_{1} & =-\left(1-p_{1}\right)(1-q) \int_{0}^{1}\left[Q_{2}\left(p_{2}\right)-p_{2} F_{2}\left(p_{2}\right)\right] d \Phi\left(p_{2}\right) \\
& <\left(1-p_{1}\right)(1-q) \bar{p}_{2} v^{\prime-1}\left(u^{\prime}(y+g)\right)
\end{aligned}
$$

where $\bar{p}_{2}:=\int_{0}^{1} p_{2} d \Phi\left(p_{2}\right)$ is the expected period-2 mortality risk. Moreover, from Remark $1, Q_{1}-$ $p_{1} F_{1} \geq 0$. Therefore,

$$
0 \leq Q_{1}-p_{1} F_{1}<\left(1-p_{1}\right)(1-q) \bar{p}_{2} v^{\prime-1}\left(u^{\prime}(y+g)\right)
$$

Taking limits on all sides of (A1) as $q \uparrow 1$ yields,

$$
0 \leq \lim _{q \uparrow 1}\left(Q_{1}-p_{1} F_{1}\right) \leq \lim _{q \uparrow 1}(1-p)(1-q) \bar{p}_{2} v^{\prime-1}\left(u^{\prime}(y+g)\right)=0 .
$$

This implies instantly that $\lim _{q \uparrow 1} F_{1}=F_{1}^{F I}$ and $\lim _{q \uparrow 1} Q_{1}=Q_{1}^{F I}$, where $\left(Q_{1}^{F I}, F_{1}^{F I}\right)$ is the solution to the following pair of equations:

$$
\begin{aligned}
u^{\prime}\left(y-g-Q_{1}^{F I}\right) & =v^{\prime}\left(F_{1}^{F I}\right) \\
p_{1} F_{1}^{F I}-Q_{1}^{F I} & =0
\end{aligned}
$$

To complete the proof, notice that

$$
(1-\tilde{q}) u^{\prime}(y+g)<(1-\tilde{q}) v^{\prime}\left(F_{2}\left(p_{2}\right)\right)=(1-q) v^{\prime}\left(F_{1}\right)+\frac{\lambda\left(p_{2}\right)}{\left(1-p_{1}\right) \phi\left(p_{2}\right)} \leq(1-q) v^{\prime}\left(F_{1}\right)
$$


where the equality follows from $7 \mathrm{~b}$ and $7 \mathrm{~d})$. Therefore,

$$
(1-\tilde{q}) u^{\prime}(y+g)=\lim _{q \uparrow 1}(1-\tilde{q}) u^{\prime}(y+g) \leq \lim _{q \uparrow 1}(1-q) v^{\prime}\left(F_{1}\right)=0 \times v^{\prime}\left(F_{1}^{F I}\right)=0,
$$

a contradiction.

Lemma A4 Fixing $\tilde{q}$, there exists a threshold $q_{0}(\tilde{q}) \in(\tilde{q}, 1)$ such that $Q_{2}\left(p_{2}\right)=0$ for all $p_{2} \in(0,1]$ if $q>q_{0}(\tilde{q})$, and $Q_{2}\left(p_{2}\right)>0$ for all $p_{2} \in(0,1]$ if $q<q_{0}(\tilde{q})$. Moreover, $q_{0}(\tilde{q})$ is weakly increasing in $\tilde{q}$.

Proof. From Lemma A1, $Q_{2}\left(p_{2}\right)>0$ for all $p_{2} \in(0,1]$ if $\Delta=0$, or equivalently $q=\tilde{q}$. Lemma A3 together with Lemma 2 indicates that there exists $q \in(\tilde{q}, 1)$ such that $Q_{2}\left(p_{2}\right)>0$ for all $p_{2} \in(0,1]$.

We first prove the existence of threshold $q_{0}(\tilde{q})$. Suppose to the contrary that there exist $q^{\prime}$ and $q$ with $q^{\prime}>q$ such that $Q_{2}\left(p_{2}\right)=0<Q_{2}^{\prime}\left(p_{2}\right)$ for all $p_{2} \in(0,1]$. It follows immediately that $\lambda\left(p_{2}\right)=0$, $\lambda^{\prime}\left(p_{2}\right) \leq 0, \gamma\left(p_{2}\right) \geq 0$, and $\gamma^{\prime}\left(p_{2}\right)=0$ for all $p_{2} \in(0,1]$. From the first order conditions $\left.7 \mathrm{c}\right)$ and $7 \mathrm{~d}$, we have,

$$
\begin{aligned}
v^{\prime}\left(F_{2}\left(p_{2}\right)\right) & =u^{\prime}\left(y+g-Q_{2}\left(p_{2}\right)\right)-\frac{1}{1-\tilde{q}} \times \frac{\gamma\left(p_{2}\right)}{\left(1-p_{1}\right) \phi\left(p_{2}\right)} \\
& <u^{\prime}\left(y+g-Q_{2}^{\prime}\left(p_{2}\right)\right) \\
& =v^{\prime}\left(F_{2}^{\prime}\left(p_{2}\right)\right), \text { for all } p_{2} \in(0,1] .
\end{aligned}
$$

The inequality follows from the fact that $\gamma\left(p_{2}\right) \geq 0$ and $Q_{2}^{\prime}\left(p_{2}\right)>0=Q_{2}\left(p_{2}\right)$; and the last equality follows from $\gamma^{\prime}\left(p_{2}\right)=0$. Therefore, $F_{2}\left(p_{2}\right)>F_{2}^{\prime}\left(p_{2}\right)$ for all $p_{2} \in(0,1]$ from the strict concavity of $v(\cdot)$. Combining the first order conditions $7 \mathrm{~b}$ and $7 \mathrm{c}$ yields,

$$
\begin{aligned}
v^{\prime}\left(F_{1}\right) & =\frac{1-\tilde{q}}{1-q} u^{\prime}\left(y+g-Q_{2}\left(p_{2}\right)\right)-\frac{\lambda\left(p_{2}\right)+\gamma\left(p_{2}\right)}{\left(1-p_{1}\right)(1-q) \phi\left(p_{2}\right)} \\
& <\frac{1-\tilde{q}}{1-q^{\prime}} u^{\prime}\left(y+g-Q_{2}^{\prime}\left(p_{2}\right)\right)-\frac{\lambda^{\prime}\left(p_{2}\right)+\gamma^{\prime}\left(p_{2}\right)}{\left(1-p_{1}\right)\left(1-q^{\prime}\right) \phi\left(p_{2}\right)}=v^{\prime}\left(F_{1}^{\prime}\right),
\end{aligned}
$$

where inequality follows from $q<q^{\prime}, Q_{2}\left(p_{2}\right)=0<Q_{2}^{\prime}\left(p_{2}\right)$, and $\gamma\left(p_{2}\right)+\gamma\left(p_{2}\right) \geq 0 \geq \gamma^{\prime}\left(p_{2}\right)+\gamma^{\prime}\left(p_{2}\right)$. Therefore, $F_{1}>F_{1}^{\prime}$ and $Q_{1}<Q_{1}^{\prime}$. Hence,

$$
\begin{aligned}
0 & =\left(Q_{1}^{\prime}-p_{1} F_{1}^{\prime}\right)+\left(1-p_{1}\right)\left(1-q^{\prime}\right) \int_{0}^{1}\left[Q_{2}^{\prime}\left(p_{2}\right)-p_{2} F_{2}^{\prime}\left(p_{2}\right)\right] d \Phi\left(p_{2}\right) \\
& >\left(Q_{1}-p_{1} F_{1}\right)+\left(1-p_{1}\right)(1-q) \int_{0}^{1}\left[Q_{2}\left(p_{2}\right)-p_{2} F_{2}\left(p_{2}\right)\right] d \Phi\left(p_{2}\right)=0
\end{aligned}
$$

which is a contradiction.

Next, we show that $q_{0}(\tilde{q})$ is weakly increasing in $\tilde{q}$. Suppose to the contrary that there exist $\tilde{q}_{1}$ and $\tilde{q}_{2}$ such that $\tilde{q}_{1}>\tilde{q}_{2}$ and $q_{0}\left(\tilde{q}_{1}\right)<q_{0}\left(\tilde{q}_{2}\right)$, then $\tilde{q}_{1}<q_{0}\left(\tilde{q}_{1}\right)$ follows directly from Lemma A4. 
Therefore, $\tilde{q}_{2}<\tilde{q}_{1}<q_{0}\left(\tilde{q}_{1}\right)<q_{0}\left(\tilde{q}_{2}\right)$. Let

$$
\dot{q}:=\frac{q_{0}\left(\tilde{q}_{1}\right)+q_{0}\left(\tilde{q}_{2}\right)}{2} \in\left(q_{0}\left(\tilde{q}_{1}\right), q_{0}\left(\tilde{q}_{2}\right)\right) .
$$

Fix $q=\dot{q}$. Because $\dot{q}<q_{0}\left(\tilde{q}_{2}\right)$, all period-2 premiums except health state $p_{2}=0$ are positive if $\tilde{q}=\tilde{q}_{2}$. By the same argument, the period-2 premiums are zero if $\tilde{q}=\tilde{q}_{1}$ because $\dot{q}>q_{0}\left(\tilde{q}_{1}\right)$. Therefore, it follows instantly that $\frac{\dot{q}-\tilde{q}_{1}}{\dot{q}}>\frac{\dot{q}-\tilde{q}_{2}}{\dot{q}}$ from Lemma A2, or equivalently $\tilde{q}_{1}<\tilde{q}_{2}$, which is a contradiction against the postulated $\tilde{q}_{2}<\tilde{q}_{1}$. This completes the proof.

Now we can prove Lemma 4 . Define $\bar{q}$ as $\bar{q}:=q_{0}(0)$. We consider two cases depending on $q$ relative to $\bar{q}$.

Case I: $q<\bar{q} \equiv q_{0}(0)$. It suffices to show that $Q_{2}\left(p_{2}\right)>0$ for all $p_{2} \in(0,1]$ and $\Delta \in[0,1]$. Suppose to the contrary that there exists $\tilde{q} \in[0,1]$ such that $Q_{2}\left(p_{2}\right)=0$ for some $p_{2} \in(0,1]$. Then $Q_{2}\left(p_{2}\right)=0$ for all $p_{2} \in(0,1]$ from Lemma 2. It follows immediately that $q \geq q_{0}(\tilde{q}) \geq q_{0}(0) \equiv \bar{q}$ from Lemma A4, a contradiction.

Case II: $q>\bar{q} \equiv q_{0}(0)$. For $\tilde{q}=0$ (i.e., $\Delta=1$ ), it follows instantly that $Q_{2}\left(p_{2}\right)=0$ for all $p_{2} \in(0,1]$ from Lemma A4. Similarly, Lemma A1 implies that $Q_{2}\left(p_{2}\right)>0$ for all $p_{2} \in(0,1]$ if $\tilde{q}=q$ (i.e., $\Delta=0$ ). Therefore, it follows from Lemma A2 that there exists a threshold $\bar{\Delta}(q) \in(0,1)$ such that $Q_{2}\left(p_{2}\right)=0$ for all $p_{2} \in(0,1]$ if $\Delta>\bar{\Delta}(q)$, and $Q_{2}\left(p_{2}\right)>0$ for all $p_{2} \in(0,1]$ if $\Delta<\bar{\Delta}(q)$. This completes the proof.

\section{Proof of Proposition 2}

Proof. For notational convenience, we use the hat symbol to refer to the variables when the degree of consumer overconfidence is $\hat{\Delta}$. We first show that it must be the case that $\hat{Q}_{1}<Q_{1}$. Suppose to the contrary that $\hat{\Delta}<\Delta$ (i.e., $\hat{\tilde{q}}>\tilde{q})$ and $\hat{Q}_{1} \geq Q_{1}$. Equation 9 implies that $\hat{F}_{1} \leq F_{1}$.

We first show that it must be the case that $\hat{Q}_{2}\left(p_{2}\right)-p_{2} \hat{F}_{2}\left(p_{2}\right) \geq Q_{2}\left(p_{2}\right)-p_{2} F_{2}\left(p_{2}\right)$ for all $p_{2} \in$ $(0,1]$. It is clear that if health state $p_{2} \in \mathcal{B}$ under $\hat{\Delta}$, then $\hat{Q}_{2}\left(p_{2}\right)-p_{2} \hat{F}_{2}\left(p_{2}\right)=0 \geq Q_{2}\left(p_{2}\right)-p_{2} F_{2}\left(p_{2}\right) ;$ and it remains to consider the case where $p_{2} \in \mathcal{N B}$ under $\hat{\Delta}$. By definition, $\hat{\lambda}\left(p_{2}\right)=0$. In addition, from the first order conditions $(7 \mathrm{~b})$ and $(7 \mathrm{~d})$ we must have

$$
\begin{aligned}
(1-\tilde{q}) v^{\prime}\left(F_{2}\left(p_{2}\right)\right) & =(1-q) v^{\prime}\left(F_{1}\right)+\frac{\lambda\left(p_{2}\right)}{\left(1-p_{1}\right) \phi\left(p_{2}\right)} \\
& \leq(1-q) v^{\prime}\left(\hat{F}_{1}\right)+\frac{\hat{\lambda}\left(p_{2}\right)}{\left(1-p_{1}\right) \phi\left(p_{2}\right)}=(1-\hat{\tilde{q}}) v^{\prime}\left(\hat{F}_{2}\left(p_{2}\right)\right)<(1-\tilde{q}) v^{\prime}\left(\hat{F}_{2}\left(p_{2}\right)\right),
\end{aligned}
$$

where the first inequality follows from $\hat{F}_{1} \leq F_{1}$ and $\lambda\left(p_{2}\right) \leq 0=\hat{\lambda}\left(p_{2}\right)$; and the second inequality follows from the postulated $\hat{\tilde{q}}>\tilde{q}$. Therefore, $\hat{F}_{2}\left(p_{2}\right)<F_{2}\left(p_{2}\right)$ if $p_{2} \in \mathcal{N B}$ under $\hat{\Delta}$. Similarly, we can show that $\hat{Q}_{2}\left(p_{2}\right) \geq Q_{2}\left(p_{2}\right)$. To see this, notice that if $\gamma\left(p_{2}\right)>0$, then we have $\hat{Q}_{2}\left(p_{2}\right) \geq$ 
$Q_{2}\left(p_{2}\right)=0$. If $\gamma\left(p_{2}\right)=0$, from the first order conditions $\left.7 \mathrm{~b}\right)$ and $7 \mathrm{c}$, we must have

$$
\begin{aligned}
(1-\tilde{q}) u^{\prime}\left(y+g-Q_{2}\left(p_{2}\right)\right) & =(1-q) v^{\prime}\left(F_{1}\right)+\frac{\lambda\left(p_{2}\right)+\gamma\left(p_{2}\right)}{\left(1-p_{1}\right) \phi\left(p_{2}\right)} \\
& \leq(1-q) v^{\prime}\left(\hat{F}_{1}\right)+\frac{\hat{\lambda}\left(p_{2}\right)+\hat{\gamma}\left(p_{2}\right)}{\left(1-p_{1}\right) \phi\left(p_{2}\right)} \\
& =(1-\hat{\tilde{q}}) u^{\prime}\left(y+g-\hat{Q}_{2}\left(p_{2}\right)\right)<(1-\tilde{q}) u^{\prime}\left(y+g-\hat{Q}_{2}\left(p_{2}\right)\right),
\end{aligned}
$$

where the first inequality follows from the postulated $\lambda\left(p_{2}\right) \leq 0=\hat{\lambda}\left(p_{2}\right)$ and $\gamma\left(p_{2}\right)=0 \leq \hat{\gamma}\left(p_{2}\right)$; and the second equality follows instantly from $\hat{\tilde{q}}>\tilde{q}$. Therefore, $\hat{Q}_{2}\left(p_{2}\right) \geq Q_{2}\left(p_{2}\right)$ always holds. To summarize, $\hat{Q}_{2}\left(p_{2}\right)-p_{2} \hat{F}_{2}\left(p_{2}\right)>Q_{2}\left(p_{2}\right)-p_{2} F_{2}\left(p_{2}\right)$ if $p_{2} \in \mathcal{N B}$ under $\hat{\Delta}$.

Firm's expected profit under $\hat{\Delta}$ is,

$$
\begin{gathered}
\left(\hat{Q}_{1}-p_{1} \hat{F}_{1}\right)+\left(1-p_{1}\right)(1-q) \int_{0}^{1}\left[\hat{Q}_{2}\left(p_{2}\right)-p_{2} \hat{F}_{2}\left(p_{2}\right)\right] d \Phi\left(p_{2}\right) \\
>\left(Q_{1}-p_{1} F_{1}\right)+\left(1-p_{1}\right)(1-q) \int_{0}^{1}\left[Q_{2}\left(p_{2}\right)-p_{2} F_{2}\left(p_{2}\right)\right] d \Phi\left(p_{2}\right)=0 .
\end{gathered}
$$

where the strict inequality follows from the postulated $p_{2}^{*}<1$, a contradiction to the zero-profit condition (4).

Next, we prove that $\hat{p}_{2}^{*}>p_{2}^{*}$. Suppose instead $\hat{p}_{2}^{*} \leq p_{2}^{*}$. It follows immediately that $\hat{Q}_{2}\left(\hat{p}_{2}^{*}\right) \leq$ $Q_{2}\left(p_{2}^{*}\right)$ and $\hat{F}_{2}\left(\hat{p}_{2}^{*}\right) \geq F_{2}\left(p_{2}^{*}\right)$ from Lemma 3. Moreover, we have shown that $\hat{Q}_{1}<Q_{1}$ and $\hat{F}_{1}>F_{1}$. Therefore, firm's expected profit in equilibrium under $\hat{\Delta}$ can be bounded from above by,

$$
\begin{aligned}
& \left(\hat{Q}_{1}-p_{1} \hat{F}_{1}\right)+\left(1-p_{1}\right)(1-q)\left\{\int_{0}^{\hat{p}_{2}^{*}}\left[\hat{Q}_{2}\left(p_{2}\right)-p_{2} \hat{F}_{2}\left(p_{2}\right)\right] d \Phi\left(p_{2}\right)+\int_{\hat{p}_{2}^{*}}^{1}\left[\hat{Q}_{2}\left(p_{2}\right)-p_{2} \hat{F}_{2}\left(p_{2}\right)\right] d \Phi\left(p_{2}\right)\right\} \\
= & \left(\hat{Q}_{1}-p_{1} \hat{F}_{1}\right)+\left(1-p_{1}\right)(1-q) \int_{\hat{p}_{2}^{*}}^{1}\left[\hat{Q}_{2}\left(\hat{p}_{2}^{*}\right)-p_{2} \hat{F}_{2}\left(\hat{p}_{2}^{*}\right)\right] d \Phi\left(p_{2}\right) \\
= & \left(\hat{Q}_{1}-p_{1} \hat{F}_{1}\right)+\left(1-p_{1}\right)(1-q)\left\{\int_{\hat{p}_{2}^{*}}^{p_{2}^{*}}\left[\hat{Q}_{2}\left(\hat{p}_{2}^{*}\right)-p_{2} \hat{F}_{2}\left(\hat{p}_{2}^{*}\right)\right] d \Phi\left(p_{2}\right)+\int_{p_{2}^{*}}^{1}\left[\hat{Q}_{2}\left(\hat{p}_{2}^{*}\right)-p_{2} \hat{F}_{2}\left(\hat{p}_{2}^{*}\right)\right] d \Phi\left(p_{2}\right)\right\} \\
\leq & \left(\hat{Q}_{1}-p_{1} \hat{F}_{1}\right)+\left(1-p_{1}\right)(1-q)\left\{0+\int_{p_{2}^{*}}^{1}\left[Q_{2}\left(p_{2}^{*}\right)-p_{2} F_{2}\left(p_{2}^{*}\right)\right] d \Phi\left(p_{2}\right)\right\} \\
< & \left(Q_{1}-p_{1} F_{1}\right)+\left(1-p_{1}\right)(1-q)\left\{\int_{0}^{p_{2}^{*}}\left[Q_{2}\left(p_{2}\right)-p_{2} F_{2}\left(p_{2}\right)\right] d \Phi\left(p_{2}\right)+\int_{p_{2}^{*}}^{1}\left[Q_{2}\left(p_{2}\right)-p_{2} F_{2}\left(p_{2}\right)\right] d \Phi\left(p_{2}\right)\right\} \\
= & 0,
\end{aligned}
$$

where the first inequality follows from $\hat{Q}_{2}\left(\hat{p}_{2}^{*}\right)-p_{2} \hat{F}_{2}\left(\hat{p}_{2}^{*}\right) \leq \hat{Q}_{2}\left(\hat{p}_{2}^{*}\right)-\hat{p}_{2} \hat{F}_{2}\left(\hat{p}_{2}^{*}\right)=0$ for $p_{2} \geq \hat{p}_{2}^{*}$, and $\hat{Q}_{2}\left(\hat{p}_{2}^{*}\right)-p_{2} \hat{F}_{2}\left(\hat{p}_{2}^{*}\right) \leq Q_{2}\left(p_{2}^{*}\right)-p_{2} F_{2}\left(p_{2}^{*}\right)$; and the second inequality follows from $\hat{Q}_{1}<Q_{1}$ and $\hat{F}_{1}>F_{1}$, a contradiction to the zero-profit condition (4) under $\hat{\Delta}$. This completes the proof.

\section{Proof of Proposition 3}


Proof. Fixing $q \in(0,1)$ and $\Delta \in(0,1)$, we consider the following three cases depending on the range of $p_{2}^{*}$.

Case I: $p_{2}^{*}=1$. From Proposition 1, the equilibrium contract coincides with the spot contracts. Similar to the proof in Proposition 2 , it can be shown that decreasing $\Delta$ does not change the shape of the equilibrium contracts, and hence consumer welfare remains unchanged.

Case II: $p_{2}^{*}=0$. In this case, $Q_{2}\left(p_{2} ; q, \Delta\right)=0$ for all $p_{2} \in[0,1]$, and $F_{2}\left(p_{2} ; q, \Delta\right)$ is constant over $p_{2}$. Define $F_{2}(q, \Delta)$ as $F_{2}(q, \Delta):=F_{2}\left(p_{2} ; q, \Delta\right)$. Then the set of the equilibrium contracts is fully characterized by $\left\langle Q_{1}(q, \Delta), F_{1}(q, \Delta), F_{2}(q, \Delta)\right\rangle$, which is the solution to the following system of equations:

$$
\begin{aligned}
(1-\tilde{q}) v^{\prime}\left(F_{2}(q, \Delta)\right) & =(1-q) v^{\prime}\left(F_{1}(q, \Delta)\right), \\
v^{\prime}\left(F_{1}(q, \Delta)\right) & =u^{\prime}\left(y-g-Q_{1}(q, \Delta)\right), \\
Q_{1}(q, \Delta)-p_{1} F_{1}(q, \Delta) & =\left(1-p_{1}\right)(1-q) \bar{p}_{2} F_{2}(q, \Delta) .
\end{aligned}
$$

Taking the partial derivative of $\mathrm{A} 4$ with respect to $\Delta$ yields,

$$
\frac{d Q_{1}(q, \Delta)}{d \Delta}-p_{1} \frac{d F_{1}(q, \Delta)}{d \Delta}=\left(1-p_{1}\right)(1-q) \bar{p}_{2} \frac{d F_{2}(q, \Delta)}{d \Delta}
$$

Therefore, the partial derivative of $W(q, \Delta)$ with respect to $\Delta$ can be simplified as,

$$
\begin{aligned}
\frac{\partial W(q, \Delta)}{\partial \Delta}=- & u^{\prime}\left(y-g-Q_{1}(q, \Delta)\right) \frac{d Q_{1}(q, \Delta)}{d \Delta}+p_{1} v^{\prime}\left(F_{1}(q, \Delta)\right) \frac{d F_{1}(q, \Delta)}{d \Delta} \\
& +\left(1-p_{1}\right)(1-q) \bar{p}_{2} v^{\prime}\left(F_{2}(q, \Delta)\right) \frac{d F_{2}(q, \Delta)}{d \Delta} \\
= & v^{\prime}\left(F_{1}(q, \Delta)\right)\left(-\frac{d Q_{1}(q, \Delta)}{d \Delta}+p_{1} \frac{d F_{1}(q, \Delta)}{d \Delta}\right) \\
& +\left(1-p_{1}\right)(1-q) \bar{p}_{2} v^{\prime}\left(F_{2}(q, \Delta)\right) \frac{d F_{2}(q, \Delta)}{d \Delta} \\
=- & {\left[v^{\prime}\left(F_{1}(q, \Delta)\right)-v^{\prime}\left(F_{2}(q, \Delta)\right)\right] \times\left(\frac{d Q_{1}(q, \Delta)}{d \Delta}-p_{1} \frac{d F_{1}(q, \Delta)}{d \Delta}\right), }
\end{aligned}
$$

where the second equality follows from (A3); and the third equality follows from (A5). It follows from equation $\mathrm{A} 2$ that

$$
v^{\prime}\left(F_{1}\right)-v^{\prime}\left(F_{2}\right)=\frac{q-\tilde{q}}{1-q} v^{\prime}\left(F_{2}\right) \geq 0
$$

Moreover, Proposition 2 implies instantly that

$$
\frac{d F_{1}(q, \Delta)}{d \Delta}<0, \text { and } \frac{d Q_{1}(q, \Delta)}{d \Delta}>0
$$

Therefore, we must have $\frac{\partial W(q, \Delta)}{\partial \Delta} \leq 0$. 
Case III: $0<p_{2}^{*}<1$. It follows from Proposition 2 that $p_{2}^{*}$ is strictly decreasing in $\Delta$. Thus, there exists a one-to-one mapping between $\Delta$ and $p_{2}^{*}$. In addition, the set of equilibrium contract is pinned down once $p_{2}^{*}$ is determined. Therefore, to show $W(q, \Delta)$ is decreasing in $\Delta$ is equivalent to show that $W^{p}\left(p_{2}^{*}\right)$ is increasing in $p_{2}^{*}$, where $W^{p}\left(p_{2}^{*}\right)$ is defined as

$$
\begin{aligned}
W^{p}\left(p_{2}^{*}\right):= & {\left[u\left(y-g-Q_{1}\left(p_{2}^{*}\right)\right)+p_{1} v\left(F_{1}\left(p_{2}^{*}\right)\right)\right] } \\
& +\left(1-p_{1}\right)(1-q) \int_{0}^{p_{2}^{*}}\left[u\left(y+g-Q_{2}^{F I}\left(p_{2}\right)\right)+p_{2} v\left(F_{2}^{F I}\left(p_{2}\right)\right)\right] d \Phi\left(p_{2}\right) \\
& +\left(1-p_{1}\right)(1-q) \int_{p_{2}^{*}}^{1}\left[u\left(y+g-Q_{2}^{F I}\left(p_{2}^{*}\right)\right)+p_{2} v\left(F_{2}^{F I}\left(p_{2}^{*}\right)\right)\right] d \Phi\left(p_{2}\right) .
\end{aligned}
$$

In the above expression, $\left\langle Q_{1}\left(p_{2}^{*}\right), F_{1}\left(p_{2}^{*}\right)\right\rangle$ is the solution to the following pair of equations:

$$
\begin{aligned}
u^{\prime}\left(y-g-Q_{1}\left(p_{2}^{*}\right)\right) & =v^{\prime}\left(F_{1}\left(p_{2}^{*}\right)\right) \\
Q_{1}\left(p_{2}^{*}\right)-p_{1} F_{1}\left(p_{2}^{*}\right) & =\left(1-p_{1}\right)(1-q) \int_{p_{2}^{*}}^{1}\left[p_{2} F_{2}^{F I}\left(p_{2}^{*}\right)-Q_{2}^{F I}\left(p_{2}^{*}\right)\right] d \Phi\left(p_{2}\right) .
\end{aligned}
$$

Taking derivative of (A7) with respect to $p_{2}^{*}$ yields,

$$
\left(1-p_{1}\right)(1-q) \int_{p_{2}^{*}}^{1}\left(p_{2} \frac{d F_{2}^{F I}\left(p_{2}^{*}\right)}{d p_{2}^{*}}-\frac{d Q_{2}^{F I}\left(p_{2}^{*}\right)}{d p_{2}^{*}}\right) d \Phi\left(p_{2}\right)=-\left(p_{1} \frac{d F_{1}\left(p_{2}^{*}\right)}{d p_{2}^{*}}-\frac{d Q_{1}\left(p_{2}^{*}\right)}{d p_{2}^{*}}\right)
$$

With slight abuse of notation, we drop $p_{2}^{*}$ in $Q_{1}(\cdot), F_{1}(\cdot), Q_{2}^{F I}(\cdot)$, and $F_{2}^{F I}(\cdot)$ in what follows. Taking derivative of $W^{p}\left(p_{2}^{*}\right)$ with respect to $p_{2}^{*}$ yields,

$$
\begin{aligned}
\frac{d W^{p}\left(p_{2}^{*}\right)}{d p_{2}^{*}}= & v^{\prime}\left(F_{1}\right)\left(p_{1} \frac{d F_{1}}{d p_{2}^{*}}-\frac{d Q_{1}}{d p_{2}^{*}}\right)+\left(1-p_{1}\right)(1-q)\left[u\left(y+g-Q_{2}^{F I}\right)+p_{2} v\left(F_{2}^{F I}\right)\right] \\
& +\left(1-p_{1}\right)(1-q) \int_{p_{2}^{*}}^{1} v^{\prime}\left(F_{2}\right)\left(p_{2} \frac{d F_{2}^{F I}}{d p_{2}^{*}}-\frac{d Q_{2}^{F I}}{d p_{2}^{*}}\right) d \Phi\left(p_{2}\right) \\
& \quad-\left(1-p_{1}\right)(1-q)\left[u\left(y+g-Q_{2}^{F I}\right)+p_{2} v\left(F_{2}^{F I}\right)\right] \\
= & v^{\prime}\left(F_{1}\right)\left(p_{1} \frac{d F_{1}}{d p_{2}^{*}}-\frac{d Q_{1}}{d p_{2}^{*}}\right)+\left(1-p_{1}\right)(1-q) \int_{p_{2}^{*}}^{1} v^{\prime}\left(F_{2}\right)\left(p_{2} \frac{d F_{2}^{F I}}{d p_{2}^{*}}-\frac{d Q_{2}^{F I}}{d p_{2}^{*}}\right) d \Phi\left(p_{2}\right) \\
= & {\left[v^{\prime}\left(F_{1}\right)-v^{\prime}\left(F_{2}\right)\right] \times\left(p_{1} \frac{d F_{1}}{d p_{2}^{*}}-\frac{d Q_{1}}{d p_{2}^{*}}\right), }
\end{aligned}
$$

where the third equality follows from (A8). By the same argument as in Case II, it can be verified that $v^{\prime}\left(F_{1}\right)-v^{\prime}\left(F_{2}\right)>0$. Moreover, it follows from Proposition 2 that $p_{2}^{*}$ and $F_{1}$ are strictly decreasing in $\Delta$, and $Q_{1}$ is strictly increasing in $\Delta$. Therefore,

$$
\frac{d F_{1}}{d p_{2}^{*}}=\frac{d F_{1}}{d \Delta} / \frac{d p_{2}^{*}}{d \Delta}>0, \text { and } \frac{d Q_{1}}{d p_{2}^{*}}=\frac{d Q_{1}}{d \Delta} / \frac{d p_{2}^{*}}{d \Delta}<0,
$$


which in turn implies that $\frac{d W^{p}\left(p_{2}^{*}\right)}{d p_{2}^{*}}>0$. This completes the proof.

\section{Proof of Lemma 5}

Proof. Suppose to the contrary that there exists a tuple $(q, \Delta)$ such that $Q_{2 s}\left(p_{2}\right)=0$ for some $p_{2} \in(0,1]$. This implies that $\gamma_{s}\left(p_{2}\right) \geq 0$ and $\lambda_{s}\left(p_{2}\right)=0$. From the first order conditions (24a) and (24c), we have

$$
\begin{aligned}
& (1-\tilde{q}) u^{\prime}(y+g)+\beta \tilde{q} u^{\prime}\left(y+g+\beta V_{2 s}\left(p_{2}\right)\right) \\
= & (1-\tilde{q}) u^{\prime}\left(y+g-Q_{2 s}\left(p_{2}\right)\right)+\beta \tilde{q} u^{\prime}\left(y+g+\beta V_{2 s}\left(p_{2}\right)\right) \\
= & u^{\prime}\left(y-g-Q_{1 s}\right)+\frac{\lambda_{s}\left(p_{2}\right)+\gamma_{s}\left(p_{2}\right)}{\left(1-p_{1}\right) \phi\left(p_{2}\right)} \\
\geq & u^{\prime}\left(y-g-Q_{1 s}\right),
\end{aligned}
$$

which is a contradiction because the left hand side of the above inequality must be strictly less than the right hand side. To see this more clearly, note that

$$
(1-\tilde{q}) u^{\prime}(y+g)+\beta \tilde{q} u^{\prime}\left(y+g+\beta V_{2 s}\left(p_{2}\right)\right) \leq[1-(1-\beta) \tilde{q}] u^{\prime}(y+g)<u^{\prime}\left(y-g-Q_{1 s}\right) .
$$

This completes the proof.

\section{Proof of Proposition 5}

Proof. Suppose to the contrary that $\hat{\Delta}<\Delta$ (i.e., $\hat{\tilde{q}}>\tilde{q}$ ) and $\hat{F}_{1 s} \leq F_{1 s}$. It follows directly that $\hat{Q}_{1 s} \geq Q_{1 s}$ from equation 25$)$. Fixing a health state $p_{2} \in(0,1]$, we first compare firm's expected profits under $\Delta$ and $\hat{\Delta}$ depending on whether $p_{2} \in \mathcal{B}_{s}$ under $\hat{\Delta}$.

Case I: $p_{2} \in \mathcal{B}_{s}$ under $\hat{\Delta}$. It is clear that $\hat{Q}_{2 s}\left(p_{2}\right)-p_{2} \hat{F}_{2 s}\left(p_{2}\right)=0 \geq Q_{2 s}\left(p_{2}\right)-p_{2} F_{2 s}\left(p_{2}\right)$.

Case II: $p_{2} \in \mathcal{N B}_{s}$ under $\hat{\Delta}$. It follows directly that $\hat{\lambda}_{s}\left(p_{2}\right)=0$. From $24 \mathrm{~b}$ and $24 \mathrm{~d}$, we have

$$
\begin{aligned}
& (1-\tilde{q}) v^{\prime}\left(F_{2 s}\left(p_{2}\right)\right)+\beta \tilde{q} u^{\prime}\left(y+g+\beta V_{2 s}\left(p_{2}\right)\right) \\
= & v^{\prime}\left(F_{1 s}\right)+\frac{\lambda_{s}\left(p_{2}\right)}{\left(1-p_{1}\right) \phi\left(p_{2}\right)} \\
\leq & v^{\prime}\left(\hat{F}_{1 s}\right)+\frac{\hat{\lambda}_{s}\left(p_{2}\right)}{\left(1-p_{1}\right) \phi\left(p_{2}\right)} \\
= & (1-\hat{\tilde{q}}) v^{\prime}\left(\hat{F}_{2 s}\left(p_{2}\right)\right)+\beta \hat{\tilde{q}} u^{\prime}\left(y+g+\beta \hat{V}_{2 s}\left(p_{2}\right)\right),
\end{aligned}
$$

where the inequality follows from the postulated $\hat{F}_{1 s} \leq F_{1 s}$ and $\lambda_{s}\left(p_{2}\right) \leq 0=\hat{\lambda}_{s}\left(p_{2}\right)$. Note that condition A9 implies that $\hat{F}_{2 s}\left(p_{2}\right)<F_{2 s}\left(p_{2}\right)$. To see this, suppose to the contrary that $\hat{F}_{2 s}\left(p_{2}\right) \geq F_{2 s}\left(p_{2}\right)$. Then it follows that $\hat{Q}_{2 s}\left(p_{2}\right) \leq Q_{2 s}\left(p_{2}\right)$ from 26 , and hence $\hat{V}_{2 s}\left(p_{2}\right) \geq$ 
$V_{2 s}\left(p_{2}\right)$. Therefore,

$$
\begin{aligned}
(1-\tilde{q}) v^{\prime}\left(F_{2 s}\left(p_{2}\right)\right)+\beta \tilde{q} u^{\prime}\left(y+g+\beta V_{2 s}\left(p_{2}\right)\right) & >(1-\hat{\tilde{q}}) v^{\prime}\left(F_{2 s}\left(p_{2}\right)\right)+\beta \hat{\tilde{q}} u^{\prime}\left(y+g+\beta V_{2 s}\left(p_{2}\right)\right) \\
& \geq(1-\hat{\tilde{q}}) v^{\prime}\left(\hat{F}_{2 s}\left(p_{2}\right)\right)+\beta \hat{\tilde{q}} u^{\prime}\left(y+g+\beta \hat{V}_{2 s}\left(p_{2}\right)\right),
\end{aligned}
$$

where the first inequality follows from the postulated $\hat{\tilde{q}}>\tilde{q}$ and $v^{\prime}\left(F_{2 s}\left(p_{2}\right)\right)=u^{\prime}(y+g-$ $\left.Q_{2 s}\left(p_{2}\right)\right)>u^{\prime}\left(y+g+\beta V_{2 s}\left(p_{2}\right)\right)$; and the second inequality follows from the postulated $\hat{F}_{2 s}\left(p_{2}\right) \geq F_{2 s}\left(p_{2}\right)$ and $\hat{V}_{2 s}\left(p_{2}\right) \geq V_{2 s}\left(p_{2}\right)$, a contradiction against A9). Thus, when $p_{2} \in \mathcal{N B}_{s}$ under $\hat{\Delta}$, it must be the case that $\hat{F}_{2 s}\left(p_{2}\right)<F_{2 s}\left(p_{2}\right)$ and $\hat{Q}_{2 s}\left(p_{2}\right)>Q_{2 s}\left(p_{2}\right)$, which in turn implies that $\hat{Q}_{2 s}\left(p_{2}\right)-p_{2} \hat{F}_{2 s}\left(p_{2}\right)>Q_{2 s}\left(p_{2}\right)-p_{2} F_{2 s}\left(p_{2}\right)$.

Next we consider firm's expected profits under $\hat{\Delta}$, which can be bounded from below by,

$$
\begin{gathered}
\left(\hat{Q}_{1 s}-p_{1} \hat{F}_{1 s}\right)+\left(1-p_{1}\right) \int_{0}^{1}\left[\hat{Q}_{2 s}\left(p_{2}\right)-p_{2} \hat{F}_{2 s}\left(p_{2}\right)\right] d \Phi\left(p_{2}\right) \\
>\left(Q_{1 s}-p_{1} F_{1 s}\right)+\left(1-p_{1}\right) \int_{0}^{1}\left[Q_{2 s}\left(p_{2}\right)-p_{2} F_{2 s}\left(p_{2}\right)\right] d \Phi\left(p_{2}\right)=0,
\end{gathered}
$$

where the strict inequality comes from the observation that the set $\mathcal{N} \mathcal{B}_{s}$ under $\hat{\Delta}$ is non-empty because $\hat{p}_{2 s}^{*}<1$, a contradiction to the zero-profit condition 20 . This completes the proof.

\section{Proof of Proposition 6}

Proof. Before proving the proposition, it is useful to state two intermediate results.

Lemma A5 For all $(q, \Delta) \in[0,1) \times[0,1], p_{2 s}^{*} \geq p_{1}$.

Proof. Suppose to the contrary that there exists a tuple $(q, \Delta)$ such that $p_{2 s}^{*}<p_{1}$, then from Lemma 6 the no-lapsation condition 221) of the period-2 health state $p_{2}=p_{1}$ does not bind (i.e. $\left.\lambda_{s}\left(p_{1}\right)=0\right)$ and $Q_{2 s}\left(p_{1}\right)-p_{1} F_{2 s}\left(p_{1}\right)<0$. In addition, we have

$$
\begin{aligned}
v^{\prime}\left(F_{2 s}\left(p_{1}\right)\right)=u^{\prime}\left(y+g-Q_{2 s}\left(p_{1}\right)\right) & >(1-\tilde{q}) u^{\prime}\left(y+g-Q_{2 s}\left(p_{1}\right)\right)+\beta \tilde{q} u^{\prime}\left(y+g+\beta V_{2 s}\left(p_{1}\right)\right) \\
& =u^{\prime}\left(y-g-Q_{1 s}\right)=v^{\prime}\left(F_{1 s}\right)
\end{aligned}
$$

where the first and third equality follows from equation 25 and 26 ; the second equality follows from the first order conditions (24a) and (24d); and the inequality follows from $y+g-Q_{2 s}\left(p_{1}\right)<$ $y+g+\beta V_{2 s}\left(p_{1}\right)$ and $\beta \in[0,1)$. Therefore, we must have $y+g-Q_{2 s}\left(p_{1}\right)<y-g-Q_{1 s}$ from the strict concavity of $u(\cdot)$, or equivalently, $Q_{2 s}\left(p_{1}\right)>Q_{1 s}+2 g$; and $F_{2 s}\left(p_{1}\right)<F_{1 s}$ from the strict concavity of $v(\cdot)$. Therefore, firm's period-1 expected profits can be bounded from above by,

$$
Q_{1 s}-p_{1} F_{1 s}<\left(Q_{2 s}\left(p_{1}\right)-2 g\right)-p_{1} F_{2 s}\left(p_{1}\right) \leq-2 g<0
$$

a contradiction to 20 . 
Lemma A6 Let $p_{2 s}^{*}$ and $\hat{p}_{2 s}^{*}$ be the equilibrium threshold above which second period premiums are actuarially favorable under $\Delta$ and $\hat{\Delta}$ respectively. Fixing $q \in[0,1)$, if $p_{2 s}^{*}<1$ and $\hat{p}_{2 s}^{*}=1$, then $\Delta>\hat{\Delta}$.

Proof. For notational convenience, we use the hat symbol to refer to the variables when the degree of consumer overconfidence is $\hat{\Delta}$. To prove that $\Delta>\hat{\Delta}$, suppose to the contrary that $\Delta \leq \hat{\Delta}$, or equivalently $\tilde{q} \geq \hat{\tilde{q}}$. First, notice that $\hat{p}_{2 s}^{*}=1$ implies that the period-2 contracts with $\hat{\Delta}$ are spot contracts for all $p_{2} \in[0,1]$, and hence $\hat{Q}_{2 s}\left(p_{2}\right)=Q_{2}^{F I}\left(p_{2}\right)$ and $\hat{Q}_{1 s}=Q_{1}^{F I}$, where $Q_{1}^{F I}$ is defined in the proof of Lemma A3. Next, consider period-2 health state $p_{2}=p_{2 s}^{*}$. From the first order conditions 24a and (24c), we have

$$
(1-\hat{\tilde{q}}) u^{\prime}\left(y+g-Q_{2}^{F I}\left(p_{2 s}^{*}\right)\right)+\beta \hat{\tilde{q}} u^{\prime}(y+g) \leq u^{\prime}\left(y-g-\hat{Q}_{1 s}\right) .
$$

From Lemma 7, we have

$$
(1-\tilde{q}) u^{\prime}\left(y+g-Q_{2 s}\left(p_{2 s}^{*}\right)\right)+\beta \tilde{q} u^{\prime}\left(y+g+\beta V_{2 s}\left(p_{2 s}^{*}\right)\right)=u^{\prime}\left(y-g-Q_{1 s}\right) .
$$

Because $p_{2 s}^{*}<1$, we must have $Q_{1 s}>Q_{1}^{F I}=\hat{Q}_{1 s}$ from 20 , which in turn implies that

$$
u^{\prime}\left(y-g-Q_{1 s}\right)>u^{\prime}\left(y-g-\hat{Q}_{1 s}\right)
$$

Inequality (A12), together with A10 an A11, implies instantly that

$$
(1-\hat{\tilde{q}}) u^{\prime}\left(y+g-Q_{2}^{F I}\left(p_{2 s}^{*}\right)\right)+\beta \hat{\tilde{q}} u^{\prime}(y+g)<(1-\tilde{q}) u^{\prime}\left(y+g-Q_{2 s}\left(p_{2 s}^{*}\right)\right)+\beta \tilde{q} u^{\prime}\left(y+g+\beta V_{2 s}\left(p_{2 s}^{*}\right)\right)
$$

which is a contradiction because the left hand side of the above inequality must be no less than the right hand side. To see this, note that

$$
\begin{aligned}
(1-\hat{\tilde{q}}) u^{\prime}\left(y+g-Q_{2 s}^{F I}\left(p_{2 s}^{*}\right)\right)+\beta \hat{\tilde{q}} u^{\prime}(y+g) & \geq(1-\tilde{q}) u^{\prime}\left(y+g-Q_{2 s}^{F I}\left(p_{2 s}^{*}\right)\right)+\beta \tilde{q} u^{\prime}(y+g) \\
& =(1-\tilde{q}) u^{\prime}\left(y+g-Q_{2 s}\left(p_{2 s}^{*}\right)\right)+\beta \tilde{q} u^{\prime}\left(y+g+\beta V_{2 s}\left(p_{2 s}^{*}\right)\right),
\end{aligned}
$$

where the inequality follows from $\beta \in[0,1)$ and the postulated $\tilde{q} \geq \hat{\tilde{q}}$; and the equality follows from Lemma 7. This completes the proof.

Now we can prove Proposition 6. Lemma A5 implies that $p_{2 s}^{*}(q, \Delta) \geq p_{1}>0$, and hence rules out the possibility that $p_{2 s}^{*}(q, \Delta)=0$. Lemma A6 indicates the existence of a threshold of consumer overconfidence $\bar{\Delta} \in[0,1]$ such that $p_{2 s}^{*}(q, \Delta)<1$ for $\Delta>\bar{\Delta}$ and $p_{2 s}^{*}(q, \Delta)=1$ for $\Delta<\bar{\Delta}$. Therefore, it suffices to consider the following two cases.

Case I: $\bar{\Delta}=1$. Therefore, $p_{2 s}^{*}=1$ for all $\Delta \in[0,1]$, implying that the period-2 equilibrium contracts are spot contracts for all $p_{2} \in[0,1]$. As a result, $W_{s}(q, \Delta)$ is constant over $\Delta$.

Case II: $\bar{\Delta}<1$. If $\Delta<\bar{\Delta}$, the argument in Case I applies. If $\Delta>\bar{\Delta}$, by the implicit function theorem, $p_{2 s}^{*}(q, \Delta)$ is continuous and differentiable in both arguments. Because 
$\left\langle Q_{2 s}\left(p_{2} ; q, \Delta\right), F_{2 s}\left(p_{2} ; q, \Delta\right)\right\rangle=\left\langle Q_{2}^{F I}\left(p_{2}\right), F_{2}^{F I}\left(p_{2}\right)\right\rangle$ if $p_{2}<p_{2 s}^{*}(q, \Delta)$ from Proposition 4, the zero-profit condition 20 can be rewritten as,

$$
\left[Q_{1 s}(q, \Delta)-p_{1} F_{1 s}(q, \Delta)\right]+\left(1-p_{1}\right) \int_{p_{2 s}^{*}(q, \Delta)}^{1}\left[Q_{2 s}\left(p_{2} ; q, \Delta\right)-p_{2} F_{2 s}\left(p_{2} ; q, \Delta\right)\right] d \Phi\left(p_{2}\right)=0 .
$$

With slight abuse of notation, we drop $q$ and $\Delta$ in $Q_{1 s}(\cdot), F_{1 s}(\cdot), Q_{2 s}(\cdot), F_{2 s}(\cdot)$, and $p_{2 s}^{*}(\cdot)$ in what follows. Taking partial derivative of the above equality with respect to $\Delta$ yields,

$$
\left(\frac{\partial Q_{1 s}}{\partial \Delta}-p_{1} \frac{\partial F_{1 s}}{\partial \Delta}\right)+\left(1-p_{1}\right) \int_{p_{2 s}^{*}}^{1}\left(\frac{\partial Q_{2 s}\left(p_{2}\right)}{\partial \Delta}-p_{2} \frac{\partial F_{2 s}\left(p_{2}\right)}{\partial \Delta}\right) d \Phi\left(p_{2}\right)=0 .
$$

Similarly, taking partial derivative of $W_{s}(q, \Delta)$ with respect to $\Delta$ yields,

$$
\begin{aligned}
\frac{\partial W_{s}(q, \Delta)}{\partial \Delta}= & v^{\prime}\left(F_{1 s}\right)\left(p_{1} \frac{\partial F_{1 s}}{\partial \Delta}-\frac{\partial Q_{1 s}}{\partial \Delta}\right) \\
& +\left(1-p_{1}\right) \int_{p_{2 s}^{*}}^{1}\left[\begin{array}{c}
(1-q) v^{\prime}\left(F_{2 s}\right) \\
+\beta q u^{\prime}\left(y+g+\beta V_{2 s}\right)
\end{array}\right]\left(p_{2} \frac{\partial F_{2 s}}{\partial \Delta}-\frac{\partial Q_{2 s}}{\partial \Delta}\right) d \Phi\left(p_{2}\right) \\
= & \left(1-p_{1}\right) \int_{p_{2 s}^{*}}^{1}\left(\frac{\partial Q_{2 s}}{\partial \Delta}-p_{2} \frac{\partial F_{2 s}}{\partial \Delta}\right)\left[\begin{array}{c}
v^{\prime}\left(F_{1 s}\right)-(1-q) v^{\prime}\left(F_{2 s}\right) \\
-\beta q u^{\prime}\left(y+g+\beta V_{2 s}\right)
\end{array}\right] d \Phi\left(p_{2}\right) \\
= & \left(1-p_{1}\right)(q-\tilde{q}) \int_{p_{2 s}^{*}}^{1}\left(\frac{\partial Q_{2 s}}{\partial \Delta}-p_{2} \frac{\partial F_{2 s}}{\partial \Delta}\right)\left[\begin{array}{c}
u^{\prime}\left(y+g-Q_{2 s}\right) \\
-\beta u^{\prime}\left(y+g+\beta V_{2 s}\right)
\end{array}\right] d \Phi\left(p_{2}\right),
\end{aligned}
$$

where the second equality follows from $\mathrm{A} 13$ and the third equation follows from the fact that $(1-\tilde{q}) v^{\prime}\left(F_{2 s}\left(p_{2}\right)\right)+\beta \tilde{q} u^{\prime}\left(y+g+\beta V_{2 s}\left(p_{2}\right)\right)=\mu_{s}=v^{\prime}\left(F_{1 s}\right)$ if $p_{2} \geq p_{2 s}^{*}$. To proceed, define $x\left(p_{2}\right)$ and $y\left(p_{2}\right)$ as follows:

$$
x\left(p_{2}\right):=\frac{\partial Q_{2 s}\left(p_{2}\right)}{\partial \Delta}-p_{2} \frac{\partial F_{2 s}\left(p_{2}\right)}{\partial \Delta}
$$

and

$$
y\left(p_{2}\right):=u^{\prime}\left(y+g-Q_{2 s}\left(p_{2}\right)\right)-\beta u^{\prime}\left(y+g+\beta V_{2 s}\left(p_{2}\right)\right) .
$$

Because $Q_{2 s}\left(p_{2}\right)$ and $V_{2 s}\left(p_{2}\right)$ are both non-negative, we must have $y\left(p_{2}\right) \geq(1-\beta) u^{\prime}(y+g)>0$. Next, we divide the set $\mathcal{N B}_{s}$ into two subsets $\mathcal{N B}_{s}^{+}$and $\mathcal{N B}_{s}^{-}$depending on the sign of $x\left(p_{2}\right)$. Specifically, let

$$
\mathcal{N B}_{s}^{+}:=\left\{p_{2} \mid p_{2} \in \mathcal{N B}_{s}, x\left(p_{2}\right) \geq 0\right\}
$$

and

$$
\mathcal{N B}_{s}^{-}:=\left\{p_{2} \mid p_{2} \in \mathcal{N B}_{s}, x\left(p_{2}\right)<0\right\}
$$

Note that $\lambda_{s}\left(p_{2}\right)=\gamma_{s}\left(p_{2}\right)=0$ for $p_{2} \in \mathcal{N B}_{s}$. Combing 24b and 24c yields,

$$
(1-\tilde{q}) u^{\prime}\left(y+g-Q_{2 s}\left(p_{2}\right)\right)+\beta \tilde{q} u^{\prime}\left(y+g+\beta V_{2 s}\left(p_{2}\right)\right)=v^{\prime}\left(F_{1 s}\right) .
$$


Recall that $\tilde{q}=q(1-\Delta)$. Taking partial derivative of (A14) with respect to $\Delta$ and rearranging yields,

$$
\begin{aligned}
& q\left[u^{\prime}\left(y+g-Q_{2 s}\left(p_{2}\right)\right)-\beta u^{\prime}\left(y+g+\beta V_{2 s}\left(p_{2}\right)\right)\right] \\
= & v^{\prime \prime}\left(F_{1 s}\right) \frac{\partial F_{1 s}}{\partial \Delta}+\beta^{2} \tilde{q} u^{\prime \prime}\left(y+g+\beta V_{2 s}\left(p_{2}\right)\right)\left(\frac{\partial Q_{2 s}\left(p_{2}\right)}{\partial \Delta}-p_{2} \frac{\partial F_{2 s}\left(p_{2}\right)}{\partial \Delta}\right) \\
& +(1-\tilde{q}) u^{\prime \prime}\left(y+g-Q_{2 s}\left(p_{2}\right)\right) \frac{\partial Q_{2 s}\left(p_{2}\right)}{\partial \Delta} .
\end{aligned}
$$

Suppose that there exist two health states, denoted by $p_{i}$ and $p_{j}$, such that $p_{i} \in \mathcal{N B}_{s}^{+}$ and $p_{j} \in \mathcal{N B}_{s}^{-}$, then we must have $x\left(p_{i}\right) \geq 0>x\left(p_{j}\right)$ by definition. Next, we show that $y\left(p_{i}\right)<y\left(p_{j}\right)$. To see this, first note that 26) implies that the partial derivative of $Q_{2 s}\left(p_{2}\right)$ and $F_{2 s}\left(p_{2}\right)$ with respect to $\Delta$ must be of different signs. Therefore, it follows immediately from the postulated $x\left(p_{i}\right) \geq 0>x\left(p_{j}\right)$ that

$$
\frac{\partial Q_{2 s}\left(p_{i}\right)}{\partial \Delta} \geq 0 \geq \frac{\partial F_{2 s}\left(p_{i}\right)}{\partial \Delta}
$$

and

$$
\frac{\partial Q_{2 s}\left(p_{j}\right)}{\partial \Delta}<0<\frac{\partial F_{2 s}\left(p_{j}\right)}{\partial \Delta} .
$$

The above two inequalities, together with equation A15 and the fact that $u^{\prime \prime}(\cdot)<0$, imply that,

$$
\begin{aligned}
q y\left(p_{i}\right) & \equiv q\left[u^{\prime}\left(y+g-Q_{2 s}\left(p_{i}\right)\right)-\beta u^{\prime}\left(y+g+\beta V_{2 s}\left(p_{i}\right)\right)\right] \\
& =v^{\prime \prime}\left(F_{1 s}\right) \frac{\partial F_{1 s}}{\partial \Delta}+\beta^{2} \underbrace{\tilde{q} \underbrace{\prime \prime}\left(y+g+\beta V_{2 s}\left(p_{i}\right)\right) x\left(p_{i}\right)}_{\leq 0}+(1-\tilde{q}) \underbrace{u^{\prime \prime}\left(y+g-Q_{2 s}\left(p_{i}\right)\right) \frac{\partial Q_{2 s}\left(p_{i}\right)}{\partial \Delta}}_{\leq 0} \\
& <v^{\prime \prime}\left(F_{1 s}\right) \frac{\partial F_{1 s}}{\partial \Delta}+\beta^{2} \tilde{q} \underbrace{u^{\prime \prime}\left(y+g+\beta V_{2 s}\left(p_{j}\right)\right) x\left(p_{j}\right)}_{>0}+(1-\tilde{q}) \underbrace{u^{\prime \prime}\left(y+g-Q_{2 s}\left(p_{j}\right)\right) \frac{\partial Q_{2 s}\left(p_{j}\right)}{\partial \Delta}}_{>0} \\
& \equiv q y\left(p_{j}\right),
\end{aligned}
$$

indicating that $y\left(p_{i}\right)<y\left(p_{j}\right)$. Next, define

$$
\bar{y}:=\sup _{p_{2} \in \mathcal{N B}_{s}^{+}} y\left(p_{2}\right) \text {, and } \underline{y}:=\inf _{p_{2} \in \mathcal{N B}_{s}^{-}} y\left(p_{2}\right) .
$$

It follows immediately that $\underline{y} \geq \bar{y}>0$. Therefore, the partial derivative of $W_{s}(q, \Delta)$ with 
respect to $\Delta$ can be further simplified as,

$$
\begin{aligned}
\frac{\partial W_{s}(q, \Delta)}{\partial \Delta} & =\left(1-p_{1}\right)(q-\tilde{q}) \int_{p_{2 s}^{*}}^{1} x\left(p_{2}\right) y\left(p_{2}\right) d \Phi\left(p_{2}\right) \\
& =\left(1-p_{1}\right)(q-\tilde{q})\left(\int_{p_{2} \in \mathcal{N} \mathcal{B}_{s}^{+}} x\left(p_{2}\right) y\left(p_{2}\right) d \Phi\left(p_{2}\right)+\int_{p_{2} \in \mathcal{N} \mathcal{B}_{s}^{-}} x\left(p_{2}\right) y\left(p_{2}\right) d \Phi\left(p_{2}\right)\right) \\
& \leq\left(1-p_{1}\right)(q-\tilde{q})\left(\int_{p_{2} \in \mathcal{N} \mathcal{B}_{s}^{+}} x\left(p_{2}\right) \bar{y} d \Phi\left(p_{2}\right)+\int_{p_{2} \in \mathcal{N} \mathcal{B}_{s}^{-}} x\left(p_{2}\right) \underline{y} d \Phi\left(p_{2}\right)\right) \\
& \leq\left(1-p_{1}\right)(q-\tilde{q}) \bar{y} \int_{p_{2 s}^{*}}^{1} x\left(p_{2}\right) d \Phi\left(p_{2}\right) \\
& =(q-\tilde{q}) \bar{y}\left(p_{1} \frac{\partial F_{1 s}}{\partial \Delta}-\frac{\partial Q_{1 s}}{\partial \Delta}\right),
\end{aligned}
$$

where the last equality follows from $\mathrm{A} 13$. From Proposition 5 , we have that $p_{1} \frac{\partial F_{1 s}}{\partial \Delta}-\frac{\partial Q_{1 s}}{\partial \Delta} \leq$ 0 ; together with the fact that $q \geq \tilde{q}$ and $\bar{y}>0$, we must have $\frac{\partial W_{s}(q, \Delta)}{\partial \Delta} \leq 0$. This completes the proof.

\section{Proof of Proposition 7}

Proof. Before proving the proposition, it is useful to state several intermediary results. With slight abuse of notation, we add $q$ into $Q_{1}(\cdot), F_{1}(\cdot), Q_{2}(\cdot)$, and $F_{2}(\cdot)$ in proof of Lemma A7, Lemma A8, and Lemma A9, to emphasize the fact that the equilibrium contract depends on $(q, \tilde{q})$, or equivalently, $(q, \Delta)$.

Lemma A7 Fixing $\tilde{q}<1$, $\lim _{q \uparrow 1}\left(1-p_{1}\right)(1-q) \int_{0}^{1}\left[u\left(y+g-Q_{2}\left(p_{2}\right)\right)+p_{2} v\left(F_{2}\left(p_{2}\right)\right)\right] d \Phi\left(p_{2}\right)=0$.

Proof. It is clear that the result holds if $\lim _{c \rightarrow \infty} v(\cdot)<\infty$. Thus, it suffices to consider the case where $\lim _{c \rightarrow \infty} v(c)=\infty$. First, it follows from Lemma A4 that $Q_{2}\left(p_{2} ; q\right)=0$ for all $p_{2} \in[0,1]$ if $q>q_{0}(\tilde{q})$, where $q_{0}(\tilde{q})$ is defined in Lemma A4. Therefore, we have that

$$
\lim _{q \uparrow 1} Q_{2}\left(p_{2} ; q\right)=0, \text { and } \lim _{q \uparrow 1} u\left(y+g-Q_{2}\left(p_{2} ; q\right)\right)=u(y+g)
$$

Second, a strictly positive lower bound of $F_{2}\left(p_{2} ; q\right)$, denoted by $\kappa$, can be established. To see this, note that the first order conditions $(7 \mathrm{c})$ and $(7 \mathrm{~d})$ imply that

$$
v^{\prime}\left(F_{2}\left(p_{2} ; q\right)\right) \leq u^{\prime}\left(y-g-Q_{2}\left(p_{2} ; q\right)\right) \leq u^{\prime}\left(y-g-Q_{2}^{F I}(1)\right)
$$

where the last inequality follows from the fact that $Q_{2}\left(p_{2} ; q\right) \leq Q_{2}^{F I}\left(p_{2}\right) \leq Q_{2}^{F I}(1)$. Therefore, we have that

$$
F_{2}\left(p_{2} ; q\right) \geq v^{\prime-1}\left(u^{\prime}\left(y-g-Q_{2}^{F I}(1)\right)\right)=: \kappa .
$$


The zero-profit condition (4), together with the fact that $0 \leq Q_{1}(q)-p_{1} F_{1}(q) \leq y-g$, implies that

$$
\int_{0}^{1} F_{2}\left(p_{2} ; q\right) d \Phi\left(p_{2}\right)=\frac{Q_{1}(q)-p_{1} F_{1}(q)}{\left(1-p_{1}\right)(1-q)} \leq \frac{y-g}{\left(1-p_{1}\right)(1-q)} .
$$

Therefore, we have that

$$
\begin{aligned}
\bar{p}_{2} v(\kappa) \equiv \int_{0}^{1} p_{2} v(\kappa) \Phi\left(p_{2}\right) & \leq \int_{0}^{1} p_{2} v\left(F_{2}\left(p_{2} ; q\right)\right) d \Phi\left(p_{2}\right) \\
& \leq \int_{0}^{1}\left[v\left(p_{2} F_{2}\left(p_{2} ; q\right)+\left(1-p_{2}\right) \kappa\right)-\left(1-p_{2}\right) v(\kappa)\right] d \Phi\left(p_{2}\right) \\
& \leq v\left(\int_{0}^{1} p_{2} F_{2}\left(p_{2} ; q\right) d \Phi\left(p_{2}\right)+\int_{0}^{1}\left(1-p_{2}\right) \kappa d \Phi\left(p_{2}\right)\right)-\left(1-\bar{p}_{2}\right) v(\kappa) \\
& \leq v\left(\frac{y-g}{\left(1-p_{1}\right)(1-q)}+\left(1-\bar{p}_{2}\right) \kappa\right)-\left(1-\bar{p}_{2}\right) v(\kappa),
\end{aligned}
$$

where the second and third inequalities follow from the concavity of $v(\cdot)$; and the last inequality follows from A16. Multiplying the above inequality by $\left(1-p_{1}\right)(1-q)$ and taking limits as $q \uparrow 1$ on all sides yields,

$$
\begin{aligned}
0 & =\lim _{q \uparrow 1}\left(1-p_{1}\right)(1-q) \bar{p}_{2} v(\kappa) \\
& \leq \lim _{q \uparrow 1}\left(1-p_{1}\right)(1-q) \int_{0}^{1} p_{2} v\left(F_{2}\left(p_{2}\right)\right) d \Phi\left(p_{2}\right) \\
& \leq \lim _{q \uparrow 1}\left(1-p_{1}\right)(1-q) v\left(\frac{y-g}{\left(1-p_{1}\right)(1-q)}+\left(1-\bar{p}_{2}\right) \kappa\right)-\lim _{q \uparrow 1}\left(1-p_{1}\right)(1-q)\left(1-\bar{p}_{2}\right) v(\kappa)=0 .
\end{aligned}
$$

The last equality holds due to the assumption that $\lim _{c \rightarrow \infty} v^{\prime}(c)=0$. Specifically,

$$
\begin{aligned}
\lim _{q \uparrow 1}\left(1-p_{1}\right)(1-q) v\left(\frac{y-g}{\left(1-p_{1}\right)(1-q)}+\left(1-\bar{p}_{2}\right) \kappa\right) & =\lim _{x \downarrow 0} \frac{v\left(\frac{y-g}{x}+\left(1-\bar{p}_{2}\right) \kappa\right)}{\frac{1}{x}} \\
& =(y-g) \lim _{x \downarrow 0} v^{\prime}\left(\frac{y-g}{x}+\left(1-\bar{p}_{2}\right) \kappa\right)=0,
\end{aligned}
$$

where the second equality follows from L'Hospital rule. Therefore, we have that

$$
\lim _{q \uparrow 1}\left(1-p_{1}\right)(1-q) \int_{0}^{1} p_{2} v\left(F_{2}\left(p_{2}\right)\right) d \Phi\left(p_{2}\right)=0,
$$

which in turn implies that,

$$
\lim _{q \uparrow 1}\left(1-p_{1}\right)(1-q) \int_{0}^{1}\left[u\left(y+g-Q_{2}\left(p_{2}\right)\right)+p_{2} v\left(F_{2}\left(p_{2}\right)\right)\right] d \Phi\left(p_{2}\right)=0 .
$$

This completes the proof. 
Lemma A8 Denote the consumer equilibrium welfare in the absence of life settlement market from environment $(q, \tilde{q})$ by $W^{\dagger}(q, \tilde{q})$. If $\eta(c) \geq \alpha>1$ for all $c>0$, then $\lim _{q \uparrow 1} W^{\dagger}(q, \tilde{q})=$ $\left[u(0)+p_{1} v(0)\right]+\left(1-p_{1}\right) u(y+g)$ for all $\tilde{q} \in[0,1)$.

Proof. It is clear that $W^{\dagger}(q, \tilde{q}) \equiv W\left(q, \frac{q-\tilde{q}}{q}\right)$, where $W(\cdot)$ is defined in 18 . Fix $\tilde{q}$. It follows from Lemma A4 that $Q_{2}\left(p_{2} ; q\right)=0$ and $\lambda\left(p_{2}\right)=0$ for all $p_{2} \in(0,1]$ when $q>q_{0}(\tilde{q})$. Combing (7b) and (7d) yields,

$$
(1-\tilde{q}) v^{\prime}\left(F_{2}\left(p_{2}\right)\right)=(1-q) v^{\prime}\left(F_{1}(q)\right),
$$

which implies that $F_{2}\left(p_{2} ; q\right) \geq F_{1}(q)$ for all $p_{2} \in(0,1]$ because $\tilde{q} \leq q$. Moreover, from the postulated $\eta(c)=-\frac{v^{\prime}(c)}{c v^{\prime \prime}(c)} \geq \alpha$, we have that

$$
\frac{d c^{\frac{1}{\alpha}} v^{\prime}(c)}{d c}=\frac{1}{\alpha} v^{\prime}(c)+c v^{\prime \prime}(c) \geq 0
$$

Therefore, $c^{\frac{1}{\alpha}} v^{\prime}(c)$ is weakly increasing in $c$. Together with $F_{2}\left(p_{2} ; q\right) \geq F_{1}(q)$, it follows that

$$
\left[F_{2}\left(p_{2} ; q\right)\right]^{\frac{1}{\alpha}} \times v^{\prime}\left(F_{2}\left(p_{2} ; q\right)\right) \geq\left[F_{1}(q)\right]^{\frac{1}{\alpha}} \times v^{\prime}\left(F_{1}(q)\right) .
$$

Equation A17, together with A18, implies that,

$$
\frac{1-q}{1-\tilde{q}}=\frac{v^{\prime}\left(F_{2}\left(p_{2} ; q\right)\right)}{v^{\prime}\left(F_{1}(q)\right)} \geq\left(\frac{F_{1}}{F_{2}\left(p_{2} ; q\right)}\right)^{\frac{1}{\alpha}} .
$$

Rearranging the above inequality yields,

$$
F_{2}\left(p_{2} ; q\right) \geq F_{1}(q)\left(\frac{1-\tilde{q}}{1-q}\right)^{\alpha} .
$$

The above inequality, together with the zero-profit condition (4), implies that

$$
\begin{aligned}
p_{1} F_{1}(q)+\left(1-p_{1}\right)(1-q) \bar{p}_{2} F_{1}(q)\left(\frac{1-\tilde{q}}{1-q}\right)^{\alpha} & \leq p_{1} F_{1}(q)+\left(1-p_{1}\right)(1-q) \int_{0}^{1} p_{2} F_{2}\left(p_{2} ; q\right) d \Phi\left(p_{2}\right) \\
& =Q_{1}(q) \leq y-g
\end{aligned}
$$

where $\bar{p}_{2} \equiv \int_{0}^{1} p_{2} d \Phi\left(p_{2}\right)$. Rearranging the above inequality yields,

$$
0<F_{1}(q) \leq \frac{y-g}{p_{1}+\left(1-p_{1}\right)(1-q) \bar{p}_{2}\left(\frac{1-\tilde{q}}{1-q}\right)^{\alpha}},
$$

where $F_{1}(q)>0$ follows directly from the Inada condition on $v(\cdot)$. Taking limits as $q \uparrow 1$ on all sides of the above inequality yields,

$$
0 \leq \lim _{q \uparrow 1} F_{1}(q) \leq \lim _{q \uparrow 1} \frac{y-g}{p_{1}+\left(1-p_{1}\right)(1-q) \bar{p}_{2}\left(\frac{1-\tilde{q}}{1-q}\right)^{\alpha}}=0,
$$


which indicates that $\lim _{q \uparrow 1} F_{1}(q)=0$, and hence $\lim _{q \uparrow 1} Q_{1}(q)=y-g$ from (9). Therefore, the consumer equilibrium welfare in the limit as $q \uparrow 1$ is,

$$
\begin{aligned}
\lim _{q \uparrow 1} W^{\dagger}(q, \tilde{q}) & :=\lim _{q \uparrow 1}\left[u\left(y-g-Q_{1}(q)\right)+p_{1} v\left(F_{1}(q)\right)\right] \\
& +\left(1-p_{1}\right) \lim _{q \uparrow 1} \int_{0}^{1}\left\{(1-q)\left[u\left(y+g-Q_{2}\left(p_{2} ; q\right)\right)+p_{2} v\left(F_{2}\left(p_{2} ; q\right)\right)\right]+q u(y+g)\right\} d \Phi\left(p_{2}\right) \\
& =\left[u(0)+p_{1} v(0)\right]+\left(1-p_{1}\right) u(y+g) .
\end{aligned}
$$

This completes the proof.

Lemma A9 Denote the consumer equilibrium welfare in the presence of settlement market from environment $(q, \tilde{q})$ by $W_{s}^{\dagger}(q, \tilde{q})$. Fix $\tilde{q} \in[0,1)$. Suppose $\eta(c) \geq \alpha>1$ for all $c>0$, then there exists a threshold $\underline{q}(\tilde{q})$ such that $W_{s}^{\dagger}(q, \tilde{q})>W^{\dagger}(q, \tilde{q})$ for $q \geq \underline{q}(\tilde{q})$.

Proof. It is clear that $W_{s}^{\dagger}(q, \tilde{q}) \equiv W_{s}\left(q, \frac{q-\tilde{q}}{q}\right)$, where $W(\cdot)$ is defined in 30 . Fixing $\tilde{q}$, note that the equilibrium contract in the presence of settlement market does not depend on $q$; and we drop $q$ in $F_{1 s}(\cdot), Q_{2 s}(\cdot)$, and $V_{2 s}(\cdot)$ in what follows. It follows directly from equation (30) that

$$
\lim _{q \uparrow 1} W_{s}^{\dagger}(q, \tilde{q})=\left[u\left(y-g-Q_{1 s}\right)+p_{1} v\left(F_{1 s}\right)\right]+\left(1-p_{1}\right) \int_{0}^{1} u\left(y+g+\beta V_{2 s}\left(p_{2}\right)\right) d \Phi\left(p_{2}\right) .
$$

Because $F_{1 s}>0$ and $Q_{1 s}<y-g$, we must have that

$$
u\left(y-g-Q_{1 s}\right)+p_{1} v\left(F_{1 s}\right)>u(0)+p_{1} v(0)
$$

The above inequality, together with the fact that $u(y+g) \leq u\left(y+g+\beta V_{2 s}\left(p_{2}\right)\right)$, imply that

$$
\begin{aligned}
\lim _{q \uparrow 1} W^{\dagger}(q, \tilde{q}) & =\left[u(0)+p_{1} v(0)\right]+\left(1-p_{1}\right) u(y+g) \\
& <\left[u\left(y-g-Q_{1 s}\right)+p_{1} v\left(F_{1 s}\right)\right]+\left(1-p_{1}\right) \int_{0}^{1} u\left(y+g+\beta V_{2 s}\left(p_{2}\right)\right) d \Phi\left(p_{2}\right)=\lim _{q \uparrow 1} W_{s}^{\dagger}(q, \tilde{q}),
\end{aligned}
$$

where the first equality follows from Lemma A8.

To complete the proof, note that fixing $\tilde{q} \in[0,1)$, we have that $W^{\dagger}(\tilde{q}, \tilde{q}) \geq W_{s}^{\dagger}(\tilde{q}, \tilde{q})$ from 31 ; together with the fact that $\lim _{q \uparrow 1} W^{\dagger}(q, \tilde{q})<\lim _{q \uparrow 1} W_{s}^{\dagger}(q, \tilde{q})$, and the continuity of $W^{\dagger}(\cdot, \cdot)$ and $W_{s}^{\dagger}(\cdot, \cdot)$, there exists a threshold $\underline{q}(\tilde{q}) \in(\tilde{q}, 1)$ such that $W_{s}^{\dagger}(q, \tilde{q})>W^{\dagger}(q, \tilde{q})$ for $q \geq \underline{q}(\tilde{q})$.

Now we can prove Proposition 7 Let $\bar{q}:=\underline{q}(0)$. It follows from Lemma A9 that

$$
W_{s}(q, 1) \equiv W_{s}^{\dagger}(q, 0)>W^{\dagger}(q, 0) \equiv W(q, 1) \text { for } q \geq \underline{q}(0) \equiv \bar{q}
$$

Moreover, we have that $W_{s}(q, 0) \leq W(q, 0)$ from (31). Fix $q \geq \bar{q}$. Because $W(q, \Delta)$ and $W_{s}(q, \Delta)$ are both continuous in $\Delta$, we must have $W_{s}(q, \Delta)>W(q, \Delta)$ if $\Delta$ is sufficiently large. This completes the proof. 


\section{Proof of Lemma 8}

Proof. The first order conditions for problem with respect to $Q_{1}, F_{1}, Q_{2}^{i} F_{2}^{i}$ yield,

$$
\begin{gathered}
u^{\prime}\left(y-g-Q_{1}\right)=\mu, \\
v^{\prime}\left(F_{1}\right)=\mu, \\
u^{\prime}\left(y+g-Q_{2}^{i}\right)=\frac{\phi_{i}}{\tilde{\phi}_{i}} \mu+\frac{\lambda_{i}+\gamma_{i}}{\left(1-p_{1}\right)(1-q) \tilde{\phi}_{i}}, \\
v^{\prime}\left(F_{2}^{i}\right)=\frac{\phi_{i}}{\tilde{\phi}_{i}} \mu+\frac{\lambda_{i}}{\left(1-p_{1}\right)(1-q) \tilde{\phi}_{i}},
\end{gathered}
$$

where $\mu, \lambda_{i}$ and $\gamma_{i}$ are the Lagrange multipliers for constraint (37), (38) and (39), with $\mu>0$, $\lambda_{i} \leq 0$ and $\gamma_{i} \geq 0$ satisfying complementary slackness conditions:

$$
\begin{gathered}
\lambda_{i}\left[Q_{2}^{i}-p_{i} F_{2}^{i}\right]=0, \\
\gamma_{i} Q_{2}^{i}=0 .
\end{gathered}
$$

For the first part of the lemma, notice that $F_{2}^{H} \geq F_{2}^{F I}\left(p_{H}\right)$. Therefore, $v^{\prime}\left(F_{2}^{H}\right) \leq v^{\prime}\left(F_{2}^{F I}\left(p_{H}\right)\right)$. Similarly, we must have $F_{1} \leq F_{1}^{F I}$, where $F_{1}^{F I}$ is defined in the proof of Lemma A3. Therefore, $v^{\prime}\left(F_{1}\right) \geq v^{\prime}\left(F_{1}^{F I}\right)$. Let

$$
\bar{\Delta}_{m}:=\max \left\{1-v^{\prime}\left(F_{1}^{F I}\right) / v^{\prime}\left(F_{2}^{F I}\left(p_{H}\right)\right), 0\right\} .
$$

Then for $\Delta_{m}>\bar{\Delta}_{m}$, we must have

$$
\begin{aligned}
\frac{\lambda_{H}}{\left(1-p_{1}\right)(1-q) \tilde{\phi}_{H}}=v^{\prime}\left(F_{2}^{H}\right)-\frac{\phi_{H}}{\tilde{\phi}_{H}} \mu & =v^{\prime}\left(F_{2}^{H}\right)-\frac{1}{1-\Delta_{m}} v^{\prime}\left(F_{1}\right) \\
& \leq v^{\prime}\left(F_{2}^{F I}\left(p_{H}\right)\right)-\frac{1}{1-\Delta_{m}} v^{\prime}\left(F_{1}^{F I}\right)<0
\end{aligned}
$$

where the first equality follows from (A19d); the second equality follows from (A19b) and the definition of $\phi_{H}$ and $\tilde{\phi}_{H}$; and the last inequality follows directly from the definition of $\bar{\Delta}_{m}$. Therefore, we must have that $\lambda_{H}<0$, implying $Q_{2}^{H}=p_{H} F_{2}^{H}>0$ and hence $Q_{2}^{H}=Q_{2}^{F I}\left(p_{H}\right)$.

For the second part of the lemma, denote the solution to $u^{\prime}\left(y-g-Q_{1}\right)=v^{\prime}\left(F_{1}\right)$ and $Q_{1}-p_{1} F_{1}=$ $(y-g) / 2$ by $\left(\check{Q}_{1}, \check{F}_{1}\right)$, and let

$$
\bar{\phi}_{L}:=\min \left\{1, \frac{y-g}{2 p_{L}\left(1-p_{1}\right)(1-q) v^{\prime-1}\left(v^{\prime}(y+g)\right)}, \frac{\bar{\Delta}_{m} u^{\prime}(y+g)}{u^{\prime}\left(y-g-\check{Q}_{1}\right)-\left(1-\bar{\Delta}_{m}\right) u^{\prime}(y+g)}\right\} .
$$

Next, we show that $Q_{2}^{L}=0$ if $\Delta_{m}>\bar{\Delta}_{m}$ and $\phi_{L}<\bar{\phi}_{L}$. Suppose to the contrary that $Q_{2}^{L}>0$, then $\gamma_{L}=0$ and full-event insurance is obtained for state $p_{L}$, which in turn implies that $F_{2}^{L}=$ 
$v^{\prime-1}\left(u^{\prime}\left(y+g-Q_{2}^{L}\right)\right)<v^{\prime-1}\left(u^{\prime}(y+g)\right)$. From the zero-profit condition 37, we must have

$$
\begin{aligned}
Q_{1}-p_{1} F_{1} & =-\left(1-p_{1}\right)(1-q) \sum_{i=L, H} \phi_{i}\left[Q_{2}^{i}-p_{i} F_{2}^{i}\right] \\
& =-\left(1-p_{1}\right)(1-q) \phi_{L}\left[Q_{2}^{L}-p_{L} F_{2}^{L}\right] \\
& <-\left(1-p_{1}\right)(1-q) \phi_{L}\left[0-p_{L} v^{\prime-1}\left(u^{\prime}(y+g)\right)\right]<\frac{y-g}{2}
\end{aligned}
$$

where the second equality follows from the fact that $Q_{2}^{H}=Q_{2}^{F I}\left(p_{H}\right)$ and $F_{2}^{H}=F_{2}^{F I}\left(p_{H}\right)$ for $\Delta_{m}>\bar{\Delta}_{m}$; the first inequality follows from $Q_{2}^{L}>0$ and $F_{2}^{L}<v^{\prime-1}\left(u^{\prime}(y+g)\right)$; and the last inequality follows from $\phi_{L}<\bar{\phi}_{L}$. This implies instantly that $Q_{1}<\check{Q}_{1}$; together with the first order condition $\mathrm{A} 19 \mathrm{c}$, we must have

$$
\begin{aligned}
\frac{\lambda_{L}+\gamma_{L}}{\left(1-p_{1}\right)(1-q) \tilde{\phi}_{L}} & =u^{\prime}\left(y+g-Q_{2}^{L}\right)-\frac{\phi_{L}}{\tilde{\phi}_{L}} \mu \\
& =u^{\prime}\left(y+g-Q_{2}^{L}\right)-\frac{\phi_{L}}{\phi_{L}+\Delta_{m}\left(1-\phi_{L}\right)} u^{\prime}\left(y-g-Q_{1}\right) \\
& \geq u^{\prime}(y+g)-\frac{\phi_{L}}{\phi_{L}+\bar{\Delta}_{m}\left(1-\phi_{L}\right)} u^{\prime}\left(y-g-\check{Q}_{1}\right)>0,
\end{aligned}
$$

where the first inequality follows from $\Delta_{m}>\bar{\Delta}_{m}, Q_{2}^{L}>0$ and $Q_{1}<\check{Q}_{1}$; and the second inequality follows from $\phi_{L}<\bar{\phi}_{L}$. Because $\lambda_{L} \leq 0$, we must have $\gamma_{L}>0$. This implies instantly that $Q_{2}^{L}=0$, a contradiction to the postulated $Q_{2}^{L}>0$. This completes the proof.

\section{Proof of Lemma 9}

Proof. The proof is similar to that in Lemma 8. The first order conditions with respect to $Q_{1 s}$, $F_{1 s}, Q_{2 s}^{i} F_{2 s}^{i}$ yield,

$$
\begin{gathered}
u^{\prime}\left(y-g-Q_{1 s}\right)=\mu_{s}, \\
v^{\prime}\left(F_{1 s}\right)=\mu_{s}, \\
(1-q) u^{\prime}\left(y+g-Q_{2 s}^{i}\right)+\beta q u^{\prime}\left(y+g+\beta V_{2 s}^{i}\right)=\frac{\phi_{i}}{\tilde{\phi}_{i}} \mu_{s}+\frac{\lambda_{i s}+\gamma_{i s}}{\left(1-p_{1}\right) \tilde{\phi}_{i}} \text { for } i \in\{H, L\} \\
(1-q) v^{\prime}\left(F_{2 s}^{i}\right)+\beta q u^{\prime}\left(y+g+\beta V_{2 s}^{i}\right)=\frac{\phi_{i}}{\tilde{\phi}_{i}} \mu_{s}+\frac{\lambda_{i s}}{\left(1-p_{1}\right) \tilde{\phi}_{i}} \text { for } i \in\{H, L\} .
\end{gathered}
$$

where $\mu_{s}, \lambda_{i s}$ and $\gamma_{i s}$ are the Lagrange multipliers for constraint 41, 442 and (43), with $\mu_{s}>0$, $\lambda_{i s} \leq 0$ and $\gamma_{i s} \geq 0$ satisfying complementary slackness conditions:

$$
\begin{gathered}
\lambda_{i s}\left[Q_{2 s}^{i}-p_{i} F_{2 s}^{i}\right]=0 \text { for } i \in\{H, L\} \\
\gamma_{i s} Q_{2 s}^{i}=0 \text { for } i \in\{H, L\} .
\end{gathered}
$$

By the same argument as in the proof of Lemma 8 , we have that $F_{2 s}^{H} \geq F_{2}^{F I}\left(p_{H}\right)$, and $F_{1 s} \leq F_{1}^{F I}$, 
which in turn implies that $v^{\prime}\left(F_{2 s}^{H}\right) \leq v^{\prime}\left(F_{2}^{F I}\left(p_{H}\right)\right)$ and $v^{\prime}\left(F_{1 s}\right) \geq v^{\prime}\left(F_{1}^{F I}\right)$. Let

$$
\overline{\bar{\Delta}}_{m}:=\max \left\{1-\frac{v^{\prime}\left(F_{1}^{F I}\right)}{(1-q) v^{\prime}\left(F_{2}^{F I}\left(p_{H}\right)\right)+\beta q u^{\prime}(y+g)}, 0\right\} .
$$

For $\Delta_{m}>\overline{\bar{\Delta}}_{m}$, we have that

$$
\begin{aligned}
\frac{\lambda_{H s}}{\left(1-p_{1}\right)(1-q) \tilde{\phi}_{H}} & =(1-q) v^{\prime}\left(F_{2 s}^{H}\right)+\beta q u^{\prime}\left(y+g+\beta V_{2 s}^{H}\right)-\frac{\phi_{H}}{\tilde{\phi}_{H}} v^{\prime}\left(F_{1 s}\right) \\
& \leq(1-q) v^{\prime}\left(F_{2}^{F I}\left(p_{H}\right)\right)+\beta q u^{\prime}(y+g)-\frac{1}{1-\Delta_{m}} v^{\prime}\left(F_{1}^{F I}\right)<0,
\end{aligned}
$$

where the equality follows from (A21b), A21c), and (A21d); the first inequality follows from $V_{2 s}^{H} \geq 0$; and the second inequality follows instantly from the definition of $\overline{\bar{\Delta}}_{m}$. Therefore, $\lambda_{H s}<0$, implying that $Q_{2 s}^{H}=p_{H} F_{2 s}^{H}>0$ and hence $Q_{2 s}^{H}=Q_{2}^{F I}\left(p_{H}\right)$. The proof for $Q_{2 s}^{L}=0$ is similar to the counterpart in Lemma 8 and is omitted for brevity.

\section{Proof of Proposition 8}

Proof. Lemma 8 and Lemma 9 state that $Q_{2}^{L}=Q_{2 s}^{L}=0, Q_{2}^{H}=Q_{2 s}^{H}=Q_{2}^{F I}\left(p_{H}\right)$, and $F_{2}^{H}=F_{2 s}^{H}=$ $F_{2}^{F I}\left(p_{H}\right)$ if $\Delta_{m}$ is sufficiently large and $\phi_{L}$ is sufficiently small. Therefore, in order to calculate consumer equilibrium welfare, it remains to pin down $\left\langle Q_{1}, F_{1}, F_{2}^{L}\right\rangle$ and $\left\langle Q_{1 s}, F_{1 s}, F_{2 s}^{L}\right\rangle$.

In the absence of life settlement market, $\left\langle Q_{1}, F_{1}, F_{2}^{L}\right\rangle$ is fully characterized by the following three equations:

$$
\begin{aligned}
u^{\prime}\left(y-g-Q_{1}\right) & =v^{\prime}\left(F_{1}\right), \\
Q_{1}-p_{1} F_{1} & =\left(1-p_{1}\right)(1-q) \phi_{L} p_{L} F_{2}^{L}, \\
v^{\prime}\left(F_{2}^{L}\right) & =\frac{\phi_{L}}{\tilde{\phi}_{L}} v^{\prime}\left(F_{1}\right) .
\end{aligned}
$$

Exploiting the constant IES functional form of $u(\cdot)$ and $v(\cdot)$, it follows immediately that $Q_{1}=$ $y-g-F_{1}$. Moreover, $\left\langle F_{1}, F_{2}^{L}\right\rangle$ can be solved as follows:

$$
F_{2}^{L}=\frac{y-g}{\left(1+p_{1}\right)\left(\frac{\phi_{L}}{\tilde{\phi}_{L}}\right)^{\rho}+\left(1-p_{1}\right)(1-q) \phi_{L} p_{L}} \text {, and } F_{1}=\left(\frac{\phi_{L}}{\tilde{\phi}_{L}}\right)^{\rho} F_{2}^{L} \text {. }
$$

Therefore, consumer equilibrium welfare in the absence of life settlement market can be derived as,

$$
\begin{aligned}
W^{m}\left(\phi_{L}, \Delta_{m}\right)= & {\left[u\left(y-g-Q_{1}\right)+p_{1} v\left(F_{1}\right)\right] } \\
& +\left(1-p_{1}\right) \sum_{i=L, H} \phi_{i}\left\{(1-q)\left[u\left(y+g-Q_{2}^{i}\right)+p_{i} v\left(F_{2}^{i}\right)\right]+q u(y+g)\right\} \\
= & \left(1+p_{1}\right) v\left(F_{1}\right)+\left(1-p_{1}\right) \phi_{L}(1-q) p_{L} v\left(F_{2}^{L}\right)+\mathcal{M},
\end{aligned}
$$


where

$$
\begin{aligned}
\mathcal{M}:= & \left(1-p_{1}\right) \phi_{L} u(y+g) \\
& +\left(1-p_{1}\right) \phi_{H}\left\{(1-q)\left[u\left(y+g-Q_{2}^{F I}\left(p_{H}\right)\right)+p_{H} v\left(F_{2}^{F I}\left(p_{H}\right)\right)\right]+q u(y+g)\right\} .
\end{aligned}
$$

Similarly, in the presence of life settlement market, $\left\langle Q_{1 s}, F_{1 s}, F_{2 s}^{L}\right\rangle$ is fully characterized by the following three equations:

$$
\begin{aligned}
u^{\prime}\left(y-g-Q_{1 s}\right) & =v^{\prime}\left(F_{1 s}\right), \\
Q_{1 s}-p_{1} F_{1 s} & =\left(1-p_{1}\right) \phi_{L} p_{L} F_{2 s}^{L}, \\
(1-q) v^{\prime}\left(F_{2 s}^{L}\right)+\beta q u^{\prime}\left(y+g+\beta V_{2 s}^{L}\right) & =\frac{\phi_{L}}{\tilde{\phi}_{L}} v^{\prime}\left(F_{1 s}\right) .
\end{aligned}
$$

Exploiting the constant IES functional form of $u(\cdot)$ and $v(\cdot)$, it follows immediately that $Q_{1 s}=$ $y-g-F_{1 s}$. Moreover, $\left\langle F_{1 s}, F_{2 s}^{L}\right\rangle$ can be solved as follows:

$$
F_{2 s}^{L}=\frac{y-g}{\left(1+p_{1}\right)\left(\frac{\phi_{L}}{\tilde{\phi}_{L}} \times \frac{1}{1-q}\right)^{\rho}+\left(1-p_{1}\right)(1-q) \phi_{L} p_{L}}, \text { and } F_{1 s}=\left(\frac{\phi_{L}}{\tilde{\phi}_{L}} \times \frac{1}{1-q}\right)^{\rho} F_{2 s}^{L} \text {. }
$$

Therefore, consumer equilibrium welfare in the presence of life settlement market can be derived as,

$$
\begin{aligned}
W_{s}^{m}\left(\phi_{L}, \Delta_{m}\right)= & {\left[u\left(y-g-Q_{1 s}\right)+p_{1} v\left(F_{1 s}\right)\right] } \\
& +\left(1-p_{1}\right) \sum_{i=L, H} \phi_{i}\left\{(1-q)\left[u\left(y+g-Q_{2 s}^{i}\right)+p_{i} v\left(F_{2 s}^{i}\right)\right]+q u(y+g)\right\} \\
= & \left(1+p_{1}\right) v\left(F_{1 s}\right)+\left(1-p_{1}\right) \phi_{L}(1-q) p_{L} v\left(F_{2 s}^{L}\right)+\mathcal{M} .
\end{aligned}
$$

Carrying out the algebra, we see that

$$
\begin{aligned}
& W^{m}\left(\phi_{L}, \Delta_{m}\right)-W_{s}^{m}\left(\phi_{L}, \Delta_{m}\right) \\
= & {\left[\left(1+p_{1}\right) v\left(F_{1 s}\right)+\left(1-p_{1}\right) \phi_{L}(1-q) p_{L} v\left(F_{2 s}^{L}\right)\right]-\left[\left(1+p_{1}\right) v\left(F_{1}\right)+\left(1-p_{1}\right) \phi_{L}(1-q) p_{L} v\left(F_{2}^{L}\right)\right] } \\
= & v\left(F_{2 s}^{L}\right)\left[\left(1+p_{1}\right)\left(\frac{\phi_{L}}{\tilde{\phi}_{L}} \times \frac{1}{1-q}\right)^{\rho-1}+\left(1-p_{1}\right) \phi_{L}(1-q) p_{L}\right] \\
& -v\left(F_{2}^{L}\right)\left[\left(1+p_{1}\right)\left(\frac{\phi_{L}}{\tilde{\phi}_{L}}\right)^{\rho-1}+\left(1-p_{1}\right) \phi_{L}(1-q) p_{L}\right] .
\end{aligned}
$$


It can be verified that $W_{s}^{m}\left(\phi_{L}, \Delta_{m}\right)>W^{m}\left(\phi_{L}, \Delta_{m}\right)$ is equivalent to

$$
\left[\frac{\left(1+p_{1}\right)\left(\frac{\phi_{L}}{\tilde{\phi}_{L}} \times \frac{1}{1-q}\right)^{\rho}+\left(1-p_{1}\right)(1-q) \phi_{L} p_{L}}{\left(1+p_{1}\right)\left(\frac{\phi_{L}}{\tilde{\phi}_{L}}\right)^{\rho}+\left(1-p_{1}\right)(1-q) \phi_{L} p_{L}}\right]^{1-\frac{1}{\rho}}<\frac{\left(1+p_{1}\right)\left(\frac{\phi_{L}}{\tilde{\phi}_{L}} \times \frac{1}{1-q}\right)^{\rho-1}+\left(1-p_{1}\right) \phi_{L}(1-q) p_{L}}{\left(1+p_{1}\right)\left(\frac{\phi_{L}}{\tilde{\phi}_{L}}\right)^{\rho-1}+\left(1-p_{1}\right) \phi_{L}(1-q) p_{L}} .
$$

Therefore, it remains to show that the above inequality holds when $\phi_{L}$ is sufficiently small and $\Delta_{m}$ is sufficiently large. By continuity, it suffices to show that the above inequality holds when $\phi_{L}$ is sufficiently small and $\Delta_{m}=1$ (i.e., $\tilde{\phi}_{L}=1$ ), which is equivalent to,

$$
\left[\frac{\left(1+p_{1}\right)\left(\phi_{L} \times \frac{1}{1-q}\right)^{\rho}+\left(1-p_{1}\right)(1-q) \phi_{L} p_{L}}{\left(1+p_{1}\right)\left(\phi_{L}\right)^{\rho}+\left(1-p_{1}\right)(1-q) \phi_{L} p_{L}}\right]^{1-\frac{1}{\rho}}<\frac{\left(1+p_{1}\right)\left(\phi_{L} \times \frac{1}{1-q}\right)^{\rho-1}+\left(1-p_{1}\right) \phi_{L}(1-q) p_{L}}{\left(1+p_{1}\right)\left(\phi_{L}\right)^{\rho-1}+\left(1-p_{1}\right) \phi_{L}(1-q) p_{L}} .
$$

Case I: $1<\rho \leq 2$. From L'Hospital rule, the left hand side of A23) is approaching 1 as $\phi_{L} \downarrow 0$. Similarly, as $\phi_{L} \downarrow 0$, the right hand side of A23 is approaching

$$
\left(\frac{1}{1-q}\right)^{\rho-1}>1
$$

if $1<\rho<2$; and is equal to

$$
\frac{\frac{1+p_{1}}{1-q}+\left(1-p_{1}\right)(1-q) p_{L}}{\left(1+p_{1}\right)+\left(1-p_{1}\right)(1-q) p_{L}}>1
$$

if $\rho=2$ for all $\phi_{L} \in(0,1)$. Hence, A23 holds if $\phi_{L}$ is sufficiently small.

Case II: $\rho>2$. Denote $\frac{1-p_{1}}{1+p_{1}}(1-q) p_{L}$ by $\tau$. Then A23 is equivalent to

$$
\begin{aligned}
g\left(\phi_{L}\right):= & \log \left[\frac{\left(\phi_{L}\right)^{\rho-2}}{(1-q)^{\rho-1}}+\tau\right]-\log \left[\left(\phi_{L}\right)^{\rho-2}+\tau\right] \\
& -\frac{\rho-1}{\rho}\left\{\log \left[\frac{\left(\phi_{L}\right)^{\rho-1}}{(1-q)^{\rho}}+\tau\right]-\log \left[\left(\phi_{L}\right)^{\rho-1}+\tau\right]\right\}>0 .
\end{aligned}
$$

Carrying out the algebra, $g^{\prime}\left(\phi_{L}\right)>0$ is equivalent to

$$
\phi_{L}<\frac{p(p-2)}{(p-1)^{2}}\left[\frac{\frac{1}{(1-q)^{\rho-1}}}{\frac{\phi_{L}^{\rho-2}}{(1-q)^{\rho-1}}+\tau}-\frac{1}{\phi_{L}^{\rho-2}+\tau}\right] /\left[\frac{\frac{1}{(1-q)^{\rho}}}{\frac{\phi_{L}^{\rho-1}}{(1-q)^{\rho}}+\tau}-\frac{1}{\phi_{L}^{\rho-1}+\tau}\right] .
$$


Note that as $\phi_{L} \downarrow 0$, the right hand side of the above inequality is approaching

$$
\frac{p(p-2)}{(p-1)^{2}}\left[\frac{1}{(1-q)^{\rho-1}}-1\right] /\left[\frac{1}{(1-q)^{\rho}}-1\right]>0
$$

and the left hand side is approaching 0. Therefore, A24 holds, or equivalently, $g(\phi)$ is strictly increasing in $\phi_{L}$, if $\phi_{L}$ is small enough; together with the fact that $g(0)=0$, there exists a threshold of $\phi_{L}$ below which $g(\phi)>0$.

Case III: $\rho<1$. The proof is similar to that of Case II and is omitted. 